$$
\begin{gathered}
\text { RECEIVED } \\
\text { APR } 131999 \\
\text { OSTI }
\end{gathered}
$$

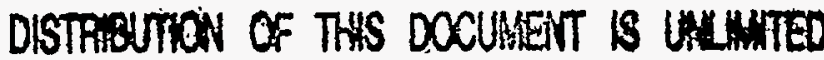

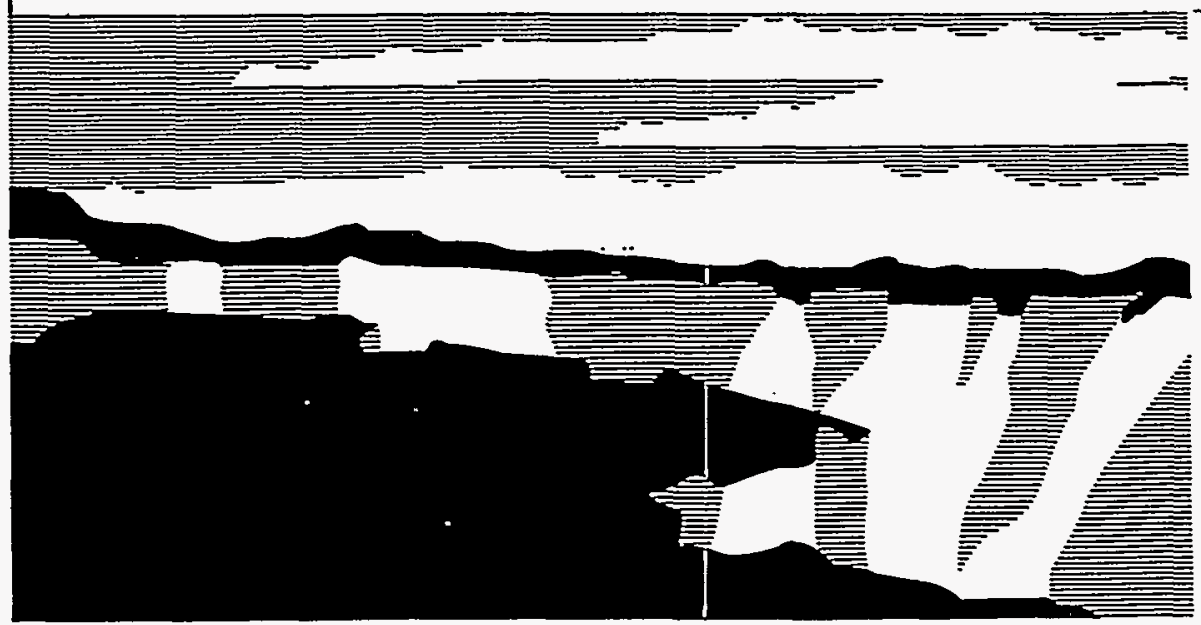

Los Alamos National Laboratory, an affimative action/equal opportunity employer, is operated by the University of Californla for the U.S. Department of Energy under contract W-7405-ENG-36. By acceptance of this article, the publisher recognizes that the U.S. Govemment retains a nonexclusive, royalty-free license to publish or reproduce the published form of this contrlbution, or to allow others to do so, for U.S. Govemment purposes. Los Alamos National Laboratory requests that the publisher Identify this anticle as work performed under the ausplces of the U.S. Department of Energy. The Los Alamos National Laboratory strongly supports acedemic freedom and a researcher's right to publish; as an institution, howover, the Laboratory does not endorse the vieupoint of a pubilcation or guaranteo its technical correctness. 


\section{DISCLAIMER}

This report was prepared as an account of work spoasared by an agency of the United States Goverament. Neither the United States Government nor any agency thereof, nor any of their employees, makes any warranty, express or implied, or assumes any legal liability or responsibility for the accuracy, completeness, or usefulness of any information, apparatus, product, or process disclosed, or represents that its use would not infringe privately owned rights. Reference herein to any specific commercial produch, process, or service by trade name, trademark, inanufacturer, or otherwise does not necessarily constitute or imply its endorsement. recommendation, or favoring by the United States Goyernment or any agency thereof. The views and opinions of authors expressed herein do not necessarily state or reflect those of the United States Government or any agency thereof. 


\section{DISCLAIMER}

Portions of this document may be illegible in electronic image products. Images are produced from the best available original document. 


\title{
THE CHARACTERISTIC ANALYSIS OF A HYBRID MULTIFLUID TURBULENT-MIX MODEL
}

\author{
BAOLIAN CHENG AND CHARLES W. CRANFILL
}

\begin{abstract}
A thorough analysis of the characteristics of a multifluid turbulent mix model in the case of one-dimensional two phase flows is presented under various physical circumstences. It has been found that the new hybrid multifluid turbulent mix model has all real characteristics if either real or turbulent viscosity is present. When real viscosity vanishes, the model still has all real charecteristics for zero relative motion between fluids. For nonzero reletive motions between fluids, the model will have all real characteristics if the disordered motions and turbulent viscosity together are generated with the nonzero relative motions simultaneously. The implications of the results are further discussed.
\end{abstract}

\section{INTRODUCTION}

It is well known that small asymmetries or disturbances in fluid systems containing multiple materials produce bouyancy- and shear-driven instabilities at the material interfaces. These instabilities lead to turbulent mixing of the materials. In the past three decades, various models of such mixing, from the simplest phenomenological model to the different variants of semiempirical models, were proposed. Among them, there were two different types of models describing such processes, one was the purely diffusive single fluid turbulence model introduced by Andronov et al ${ }^{[1,2]}$ and by Besnard, Harlow, and Rauenzahn ${ }^{[3,4]}$, the other was the purely convective multifluid interpenetration model developed by Youngs ${ }^{[5]}$. However, the flow motions in a real mixing process are early-time convective and late-time diffusive. A correct description of the fundamental mixing processes, therefore, should embody both motions. A few mix models which combine the single fluid and multifluid characterizations have been constructed ${ }^{[6-8]}$. In particular, a novel hybrid multifluid turbulent mix model was recently proposed by Cranfill ${ }^{[9,10]}$. This model innovatively provides a more natural formalism for deriving the combined multifluid turbulent mix equations by dividing the turbulent contributions to the bulk fluid properties into ordered convective and disordered diffusive parts. In this model, the ordered contributions are explicitly given as the sums of the multifluid drift motions, and summing the multifluid interpenetration equations over the mixture constituents uniquely produces the bulk fluid equations with turbulent contributions. The resulting energy cascade in this model is from bulk kinetic to ordered drift kinetic to disordered turbulent kinetic to thermal internal energy. Such exclusive features intrigue us to further examine this model mathematically by utilizing the method of characteristics.

The method of characteristics has been widely used among analysts of one dimensional unsteady flows due to its relative simplicity and the ease with which boundary conditions can be incorporated. In the view of mathematics, a model with complete physics should

Date: July $13,1998$.

LANL Paper Deaignation: Journal. 
have all real characteristics, which insures that the predictions from its governing equations are meaningful; any imaginary characteristics are associated with unphysical instabilities that frequently cause the numerical evaluation of the equations to give senseless predictions for the dynamics of the system. The occurrence and significance of complex characteristics in two-phase flow equations have been discussed extensively over the past decades. It has been realized that the imaginary characteristics are directly due to the assumptions of pressure equilibrium and no dissipation in inviscid flows. The completion of physics of the two-phase flow equations and the justification of the assumption of zero dissipation in two inviscid flows with non-zero relative velocity between phases has never been examined. Now we propose that the addition of disordered diffusive motions to the two-phase flow equations would consummate the physics missed in previous models and provide a natural dissipation for the system which will make the equations more realistic. In this report, we will perform a thorough analysis of the characteristics of the new hybrid multifluid turbulent mix model in the case of one dimensional two-phase flows under various physical situations. We will show that the physical origin of the complex characteristics in previous models is not the single pressure assumption, but rather the incomplete and improper physics incorporated in the governing equations, in particular, the neglect of the disordered motions in the dynamical equations. It is evident that if the fluids are inviscid, any relative velocity between them should inevitably generate instabilities that set up disordered motions simultaneously. However this physical phenomenon has often been omitted in the traditional so called illposed one pressure two phase flow equations. This report will show that as long as this effect is taken into account properly, the one pressure hybrid multifluid turbulence model will have real characteristics even in the inviscid limit.

This report is organized as follows: Section 2 gives a summary of the hybrid multifluid turbulent mix model. In section 3 we reduce the model to one dimensional two-phase flow as an example. A thorough analysis of the characteristics for the one-dimensional equations under various physical situations is displayed in Section 4. Finally, in Section 5 we will discuss the physical implications of our results and identify possible constraints to the model. The physical origin of the complex characteristics in the traditional one-pressure inviscid two-phase flow equations is also discussed.

\section{THE HYBRID TURBULENT MIX MODEL}

The new hybrid multifluid turbulent mix model proposed and developed by Cranfill ${ }^{[9,10]}$ is based on the usual decomposition of the fluid properties into mean and fluctuating parts whose evolution equations are obtained from the Navier-Stokes equations. Correlations among the fluctuating parts produce turbulent contributions to the bulk fluid properties. The turbulent contributions in the model are uniquely divided into ordered and disordered parts, where the ordered parts are obtained from the average drift motions produced by a set of multifluid interpenetration equations, while the disordered parts are obtained from a set of single fluid turbulence equations. In this scenario, the multifluid and the single fluid turbulence equations are naturally coupled together, which enables the model to correctly describe both the early-time convective and the late-time diffusive drift motions seen in numerical and experimental investigations of the evolution of interfacial instabilities. This provides a justifiable description of the turbulent mixing in many fluid flows of interest. The mathematical description of the model is given in the remainder of this section. 


\subsection{Nomenclature.}

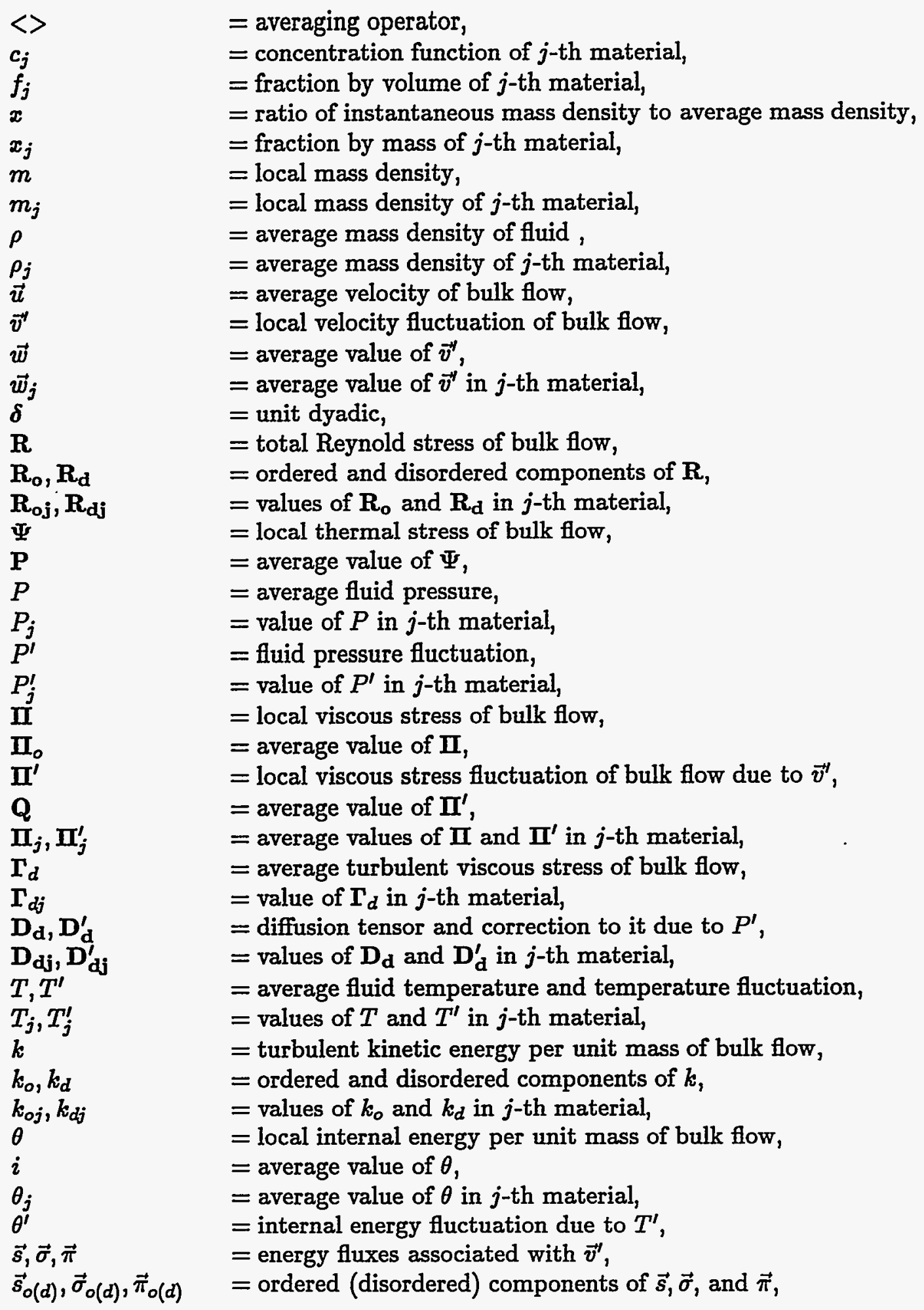




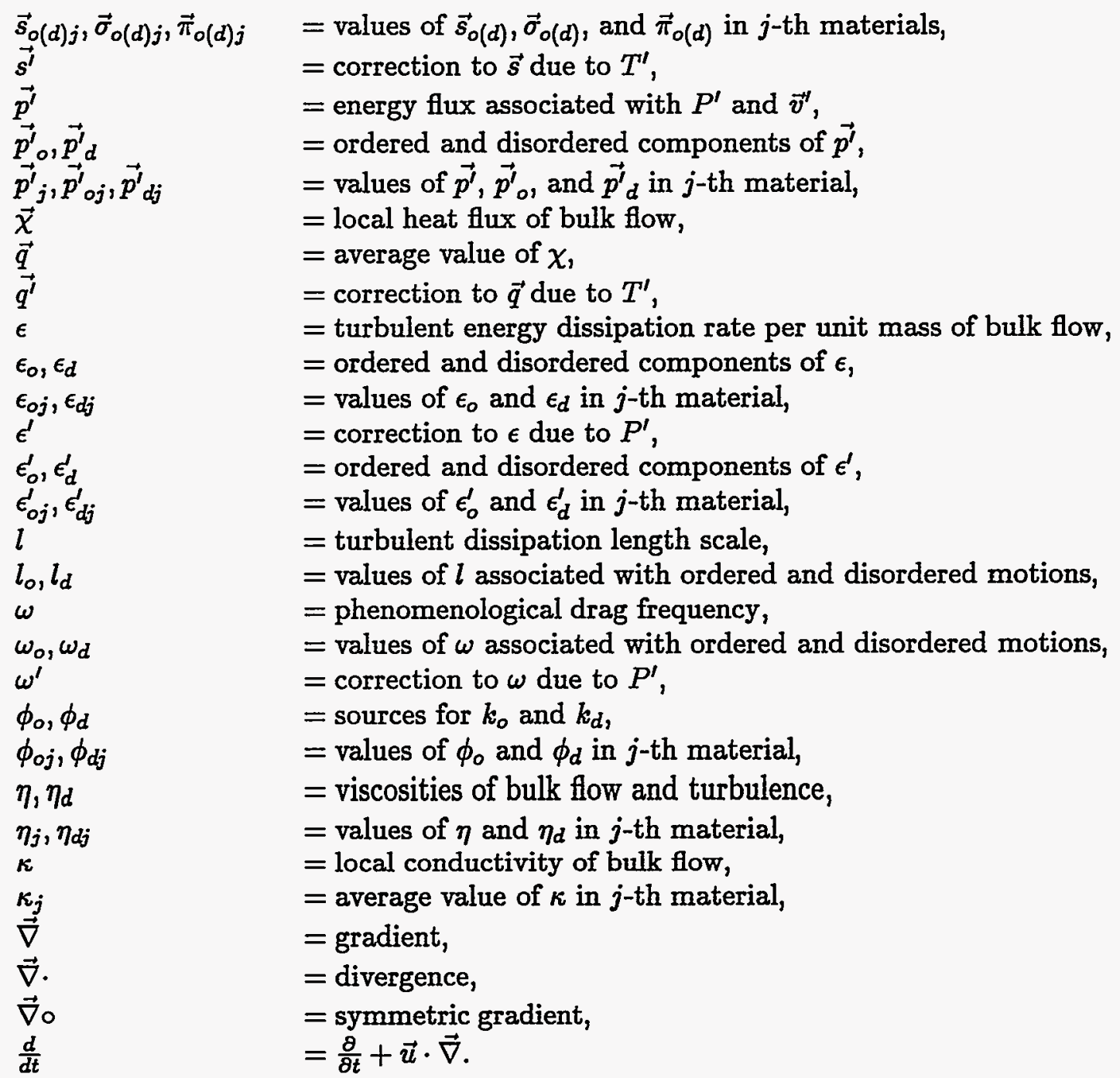

\subsection{Definitions.}

$$
\begin{gathered}
\rho=<m>, \\
\rho \vec{u}=<m \vec{v}>, \\
\vec{v}^{\prime}=\vec{v}-\vec{u}, \quad \vec{w}=<\vec{v}^{\prime}>, \\
\rho i=<m \theta>, \\
\Psi=P \delta+\Pi, \quad \mathbf{P}=<\Psi>, \\
\vec{q}=<\vec{\chi}>, \\
f_{j}=<c_{j}> \\
x_{j}=\left\langle c_{j} x>\right.
\end{gathered}
$$




$$
\begin{aligned}
& \rho_{j}=x_{j} \rho, \\
& x_{j} \vec{w}_{j}=<c_{j} x \vec{v}^{\prime}> \\
& \left.x_{j} \theta_{j}=<c_{j} x\right\rangle \text {, } \\
& f_{j} P_{j}=<c_{j} P> \\
& f_{j} T_{j}=<c_{j} T> \\
& f_{j} \Pi_{j}=<c_{j} \Pi>, \quad f_{j} \Pi_{j}^{\prime}=<c_{j} \Pi^{\prime}>, \\
& f_{j} \eta_{j}=<c_{j} \eta>\text {, } \\
& f_{j} \kappa_{j}=<c_{j} \kappa> \\
& \sum_{j} f_{j}=\sum_{j} x_{j}=1, \\
& \sum_{j} x_{j} \vec{w}_{j}=0, \quad \sum_{j} f_{j} \vec{w}_{j}=\vec{w}, \\
& \sum_{j} x_{j} \theta_{j}=i \\
& \sum_{j} f_{j} \eta_{j}=<\eta> \\
& \sum_{j} f_{j} \kappa_{j}=<\kappa> \\
& \mathbf{R}=\left\langle m \vec{v}^{\prime 2}>=\mathbf{R}_{\mathbf{o}}+\mathbf{R}_{\mathbf{d}},\right. \\
& \mathbf{R}_{\mathrm{o}}=\sum_{j} f_{j} \mathbf{R}_{\mathrm{oj}}, \quad \mathbf{R}_{\mathrm{oj}}=f_{j}^{-1} \rho \boldsymbol{x}_{j} \vec{w}_{j}^{2} \\
& \mathbf{R}_{\mathrm{d}}=\sum_{j} f_{j} \mathbf{R}_{\mathrm{dj}}, \quad \mathbf{R}_{\mathrm{dj}}=f_{j}^{-1}<c_{j} \rho x\left(\vec{v}^{\prime}-\vec{w}_{j}\right)^{2}>=\frac{2}{3} \rho k_{d} \boldsymbol{\delta}+\Gamma_{d j} \\
& \boldsymbol{\Gamma}_{d j}=-\eta_{d}\left[\vec{\nabla} \circ\left(\vec{u}+\vec{w}_{j}\right)-\frac{2}{3} \vec{\nabla} \cdot\left(\vec{u}+\vec{w}_{j}\right) \delta\right], \quad \eta_{d}=C_{\eta d} \omega^{-1}\left(\frac{2}{3} \rho k_{d}\right), \\
& \mathbf{P}=\sum_{j} f_{j}\left(P_{j} \delta+\Pi_{j}\right)=P \boldsymbol{\delta}+<\Pi> \\
& <\Pi>\equiv \sum_{j} f_{j} \Pi_{j} \equiv \Pi_{o}=-<\eta>\left(\vec{\nabla} \circ \vec{u}-\frac{2}{3} \vec{\nabla} \cdot \vec{u}\right)+\mathbf{Q}, \\
& \Pi_{j} \equiv-\eta_{j}\left[\vec{\nabla} \circ\left(\vec{u}+\vec{w}_{j}\right)-\frac{2}{3} \vec{\nabla} \cdot\left(\vec{u}+\vec{w}_{j}\right) \delta\right],
\end{aligned}
$$




$$
\begin{aligned}
& \mathbf{Q}=<\boldsymbol{\Pi}^{\prime}>=\sum_{j} f_{j} \boldsymbol{\Pi}_{j}^{\prime}, \quad \boldsymbol{\Pi}_{j}^{\prime}=-\eta_{j}\left(\vec{\nabla} \circ \vec{w}_{j}-\frac{2}{3} \vec{\nabla} \cdot \vec{w}_{j} \delta\right) \\
& \mathbf{D}_{\mathbf{d}} \equiv \omega^{-1}\left(\mathbf{R}_{\mathbf{d}}+\mathbf{\Pi}_{\mathrm{o}}\right) \equiv \sum_{j} f_{j} \mathbf{D}_{\mathbf{d} \mathbf{j}}, \quad \mathbf{D}_{\mathbf{d} \mathbf{j}} \equiv \omega^{-1}\left(\mathbf{R}_{\mathbf{d j}}+\Pi_{j}\right) \\
& \omega \rho x_{j} \vec{w}_{j} \equiv-<\Pi \cdot \vec{\nabla} c_{j}>, \quad \omega=\frac{2}{3} C_{\omega} \sqrt{k_{o}+k_{d}} / l \\
& \vec{s}=<m \theta \vec{v}^{\prime}>=\vec{s}_{o}+\vec{s}_{d}, \quad \vec{s}_{o}=\sum_{j} \rho x_{j} \theta_{j} \vec{w}_{j}, \\
& \vec{s}_{d}=\sum_{j} f_{j} \vec{s}_{d j}=\sum_{j}<c_{j} \rho x \theta\left(\vec{v}^{\prime}-\vec{w}_{j}\right)> \\
& f_{j} \vec{s}_{d j}=-f_{j} C_{i d}\left(\frac{\partial i}{\partial T}\right)_{\rho} \omega^{-1}\left(\mathbf{R}_{\mathbf{d j}}+\Pi_{j}\right) \cdot \vec{\nabla} T=-f_{j} C_{i d}\left(\frac{\partial i}{\partial T}\right)_{\rho} \mathbf{D}_{\mathbf{d j}} \cdot \vec{\nabla} T, \\
& \vec{q}=-<\kappa>\vec{\nabla} T \\
& \vec{\sigma}=<\frac{1}{2} m v^{\prime 2} \vec{v}^{\prime}>=\vec{\sigma}_{o}+\vec{\sigma}_{d}, \quad \vec{\sigma}_{o}=\sum_{j} \frac{1}{2} \rho x_{j} w_{j}^{2} \vec{w}_{j} \\
& \vec{\sigma}_{d}=\sum_{j}\left(\rho x_{j} k_{d j} \vec{w}_{j}+f_{j} \mathbf{R}_{d j} \cdot \vec{w}_{j}+f_{j} \vec{\sigma}_{d j}\right), \quad f_{j} \vec{\sigma}_{d j}=<c_{j} \frac{1}{2} \rho x\left|\vec{v}^{\prime}-\vec{w}_{j}\right|^{2}\left(\vec{v}^{\prime}-\vec{w}_{j}\right)>, \\
& \vec{\pi}=<\Pi \cdot \vec{v}^{\prime}>=\vec{\pi}_{o}+\vec{\pi}_{d}, \quad \vec{\pi}_{o}=\sum_{j} f_{j} \Pi_{j} \cdot \vec{w}_{j}, \\
& \vec{\pi}_{d}=\sum_{j} f_{j} \vec{\pi}_{d j}, \quad f_{j} \vec{\pi}_{d j}=<c_{j} \Pi \cdot\left(\vec{v}^{\prime}-\vec{w}_{j}\right)>=<c_{j} \Pi \cdot \vec{v}^{\prime}>-f_{j} \Pi_{j} \cdot \vec{w}_{j}, \\
& \vec{\sigma}_{d}+\vec{\pi}_{d}=\sum_{j}\left(\rho x_{j} k_{d j} \vec{w}_{j}+f_{j} \mathbf{R}_{d \mathbf{j}} \cdot \vec{w}_{j}\right)+\sum_{j} f_{j}\left(\vec{\sigma}_{d j}+\vec{\pi}_{d j}\right), \\
& \vec{\sigma}_{d j}+\vec{\pi}_{d j}=-C_{k d} \omega^{-1}\left(\mathbf{R}_{\mathbf{d j}}+\Pi_{j}\right) \cdot \vec{\nabla} k_{d}=-C_{k d} \mathbf{D}_{\mathbf{d j}} \cdot \vec{\nabla} k_{d} \\
& \rho k \equiv<\frac{1}{2} m v^{\prime 2}>=\rho k_{o}+\rho k_{d}, \quad \rho k_{o}=\sum_{j} \frac{1}{2} \rho x_{j} w_{j}^{2}, \\
& \rho k_{d}=\sum_{j} \rho x_{j} k_{d j} \equiv \sum_{j}<c_{j} \frac{1}{2} \rho x\left|\vec{v}^{\prime}-\vec{w}_{j}\right|^{2}>=\sum_{j} f_{j} \rho k_{d}, \\
& \rho \epsilon=\rho \epsilon_{o}+\rho \epsilon_{d}, \quad \rho \epsilon_{o}=-\sum_{j} f_{j} \Pi_{j}^{\prime}: \vec{\nabla} \vec{w}_{j}, \\
& \rho \epsilon_{d}=\sum_{j} \rho x_{j} \epsilon_{d j} \equiv-\sum_{j}<c_{j} \Pi^{\prime}: \vec{\nabla}\left(\vec{v}^{\prime}-\vec{w}_{j}\right)>=\sum_{j} f_{j} \rho \epsilon_{d}, \\
& l \equiv k_{d} \sqrt{k_{o}+k_{d}} / \epsilon_{d}
\end{aligned}
$$




$$
\rho \phi_{o}=\sum_{j} \vec{\nabla} \cdot\left(f_{j} \mathbf{R}_{\mathrm{dj}}\right) \cdot \vec{w}_{j}, \quad \rho \phi_{d}=\sum_{j} f_{j} \mathbf{R}_{\mathrm{dj}}: \vec{\nabla}\left(\vec{u}+\vec{w}_{j}\right),
$$

$C_{x y}(x y=\eta d, i d, k d, l d$, and $\omega)$ are constants adjusted to fit experiments. Subscripts "o" and " $d$ " represent the respective ordered-convective and disordered-diffusive components of a quantity.

2.3 Model Equations. The mathematical equations defining the new multifluid turbulent mix model are summarized as follows:

- Bulk fluid equations:

$$
\begin{gathered}
0=\frac{d}{d t} \rho+\rho \vec{\nabla} \cdot \vec{u}, \\
0=\rho \frac{d}{d t} \vec{u}+\vec{\nabla} \cdot(\mathbf{R}+\mathbf{P}), \\
0=\rho \frac{d}{d t} i+\vec{\nabla} \cdot(\vec{s}+\vec{q})+(\mathbf{P}+\mathbf{Q}): \vec{\nabla} \vec{u}+P \vec{\nabla} \cdot \vec{w}-\rho \epsilon .
\end{gathered}
$$

- Multifluid interpenetration equations:

$$
\begin{aligned}
& 0=\rho \frac{d}{d t} x_{j}+\vec{\nabla} \cdot\left(\rho x_{j} \vec{w}_{j}\right) \\
& 0=\rho \frac{d}{d t}\left(x_{j} \vec{w}_{j}\right)+\rho x_{j}\left(\frac{d}{d t}+\vec{w}_{j} \cdot \vec{\nabla}\right) \vec{u}+\vec{\nabla} \cdot\left(\rho x_{j} \vec{w}_{j}^{2}+f_{j} \mathbf{R}_{\mathbf{d j}}+f_{j} \Pi_{j}\right)+f_{j} \vec{\nabla} P+\omega \rho x_{j} \vec{w}_{j}, \\
& P_{j}\left(m_{j}, \theta_{j}\right)=P\left(\rho, i, x_{1}, x_{2}, \ldots\right), \quad T_{j}\left(m_{j}, \theta_{j}\right)=T\left(\rho, i, x_{1}, x_{2}, \ldots\right), \quad m_{j}=\rho x_{j} / f_{j} \\
& 0=\rho \frac{d}{d t} k+\vec{\nabla} \cdot(\vec{\sigma}+\vec{\pi})+(\mathbf{R}-\mathbf{Q}): \vec{\nabla} \vec{u}+(\vec{\nabla} P) \cdot \vec{w}+\rho \epsilon, \\
& 0=\rho \frac{d}{d t} k_{o}+\vec{\nabla} \cdot\left(\vec{\sigma}_{o}+\vec{\pi}_{o}\right)+\left(\mathbf{R}_{\mathbf{o}}-\mathbf{Q}\right): \vec{\nabla} \vec{u}+(\vec{\nabla} P) \cdot \vec{w}+\rho \phi_{o}+2 \omega \rho k_{o}+\rho \epsilon_{o}, \\
& 0=\rho \frac{d}{d t} k_{d}+\vec{\nabla} \cdot\left(\rho k_{d} \vec{w}-C_{k d} \mathbf{D}_{\mathrm{d}} \cdot \vec{\nabla} k_{d}\right)+\rho \phi_{d}-2 \omega \rho k_{o}+\rho \epsilon_{d} \\
& 0=\rho \frac{d}{d t} l+\vec{\nabla} \cdot\left(\rho l \vec{w}-C_{l d} \mathbf{D}_{\mathrm{d}} \cdot \vec{\nabla} l\right)-C_{l o} \rho\left(l \vec{\nabla} \cdot \vec{u}+\sqrt{k_{o}+k_{d}}\right) .
\end{aligned}
$$

The mixture here is assumed to be in an equilibrium with all materials having the same pressure, $P_{j}=P$, and temperature, $T_{j}=T$, so the $f_{j}$ and $\theta_{j}$ can be derived from the equations of state Eq.(53) without solving separate evolution equations for them. For given values of $\rho, i$ and the $x_{j}$, the $f_{j}, \theta_{j}, P$, and $T$ will be uniquely determined. Thus the model has evolution equations for all the independent variables $\rho, \vec{u}, i, x_{j}, \vec{w}_{j}, k_{d}$, and $l$ which describe the dynamics of the fluid system. Therefore this new hybrid multifluid turbulent mix model is mathematically complete.

If the mixture has a minute departure from both pressure and temperature equilibrium, then different materials would have different pressure and temperature fluctuations, $P_{j}^{\prime}$ and $T_{j}^{\prime}$. Under this physical circumstance, the evolution equations of the fluid system need to be modified as discussed in Appendix A. 
It is worthwhile to point out that this model only contains one drag frequency and one turbulence length scale for simplicity. In addition, it does not include mass diffusion which would allow the materials to mix at the atomic level. A real mixing process probably involves a spectrum of both $\omega$ and $l$, and the materials might mix at a microscopic scale in most fluid systems. One important advantage of this model is that its evolution equations are symmetrical in all materials and are formulated with vectors. This distinctive character allows the model to be applied to systems that contain any numbers of materials and spatial dimensions. We will apply this model to a one-dimensional two-fluid system as an example in the next section.

\section{ONE-DIMENSIONAL TWO-FLUID SYSTEM}

3.1 General theory. In a one-dimensional system consisting of only two fluids, the evolution equations are obtained directly from the new multifluid turbulent mix model presented in Section 2 by setting the material index $j=1$ and 2. For convenience, we replace the two material values of each physical quantity with their volume average and difference as follows:

$$
\begin{aligned}
& w \equiv f_{1} w_{1}+f_{2} w_{2}=\left(f_{1}-x_{1}\right) \delta w, \delta w \equiv w_{1}-w_{2}, w_{1}=x_{2} \delta w, w_{2}=-x_{1} \delta w \\
& \eta \equiv f_{1} \eta_{1}+f_{2} \eta_{2}, \delta \eta=\eta_{1}-\eta_{2}, \eta_{1}=\eta+f_{2} \delta \eta, \eta_{2}=\eta-f_{1} \delta \eta \\
& \Gamma_{d}=f_{1} \Gamma_{d 1}+f_{2} \Gamma_{d 2}, \delta \Gamma_{d}=\Gamma_{d 1}-\Gamma_{d 2}, \Gamma_{d 1}=\Gamma_{d}+f_{2} \delta \Gamma_{d}, \Gamma_{d 2}=\Gamma_{d}-f_{1} \delta \Gamma_{d}
\end{aligned}
$$

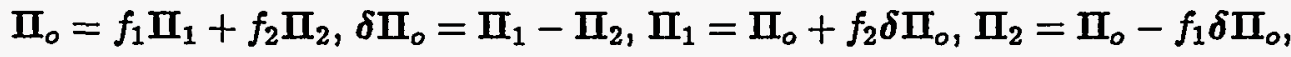

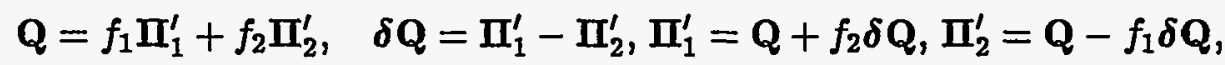

$$
\begin{aligned}
& \Phi=\Pi_{0}+\Gamma_{d}, \delta \Phi=\delta \Pi_{0}+\delta \Gamma_{d,} \\
& \mathbf{R}_{\mathrm{d}}=f_{1} \mathbf{R}_{\mathbf{d} 1}+f_{2} \mathbf{R}_{\mathbf{d} 2}, \delta \mathbf{R}_{\mathbf{d}}=\mathbf{R}_{\mathbf{d} 1}-\mathbf{R}_{\mathbf{d} 2}, \mathbf{R}_{\mathbf{d} 1}=\mathbf{R}_{\mathbf{d}}+f_{2} \delta \mathbf{R}_{\mathbf{d}}, \mathbf{R}_{\mathbf{d} 2}=\mathbf{R}_{\mathbf{d}}-f_{1} \delta \mathbf{R}_{\mathbf{d}} \\
& i=x_{1} \theta_{1}+x_{2} \theta_{2}, \delta \theta=\theta_{1}-\theta_{2}, \theta_{1}=i+x_{2} \delta \theta, \theta_{2}=i-x_{1} \delta \theta, \\
& \frac{\partial w_{1}}{\partial x}=-\delta w \frac{\partial x_{1}}{\partial x}+x_{2} \frac{\partial \delta w}{\partial x}, \quad \frac{\partial w_{1}}{\partial t}=-\delta w \frac{\partial x_{1}}{\partial t}+x_{2} \frac{\partial \delta w}{\partial t}, \\
& \frac{\partial w_{2}}{\partial x}=-\delta w \frac{\partial x_{1}}{\partial x}-x_{1} \frac{\partial \delta w}{\partial x}, \quad \frac{\partial w_{2}}{\partial t}=-\delta w \frac{\partial x_{1}}{\partial t}-x_{1} \frac{\partial \delta w}{\partial t}, \\
& \frac{\partial w}{\partial x}=\delta w \frac{\partial f_{1}}{\partial x}-\delta w \frac{\partial x_{1}}{\partial x}+\left(f_{1}-x_{1}\right) \frac{\partial \delta w}{\partial x}, \quad \frac{\partial w}{\partial t}=\delta w \frac{\partial f_{1}}{\partial t}-\delta w \frac{\partial x_{1}}{\partial t}+\left(f_{1}-x_{1}\right) \frac{\partial \delta w}{\partial t} \\
& \boldsymbol{\Gamma}_{d}=\frac{2}{3} \Gamma_{d}\left(\delta-3 \hat{\mathrm{x}}^{2}\right), \quad \Gamma_{d}=\eta_{d}\left[\frac{\partial u}{\partial x}-\delta w \frac{\partial x_{1}}{\partial x}+\left(f_{1}-x_{1}\right) \frac{\partial \delta w}{\partial x}\right]
\end{aligned}
$$


THE CHARACTERISTIC ANALYSIS OF A HYBRID MULTIFLUID TURBULENT-MIX MODEL

$$
\Gamma_{d 1}=\frac{2}{3} \Gamma_{d 1}\left(\delta-3 \hat{\mathbf{x}}^{2}\right), \quad \Gamma_{d 1}=\Gamma_{d}+f_{2} \delta \Gamma_{d}
$$

$$
\Gamma_{d 2}=\frac{2}{3} \Gamma_{d 2}\left(\delta-3 \hat{\mathbf{x}}^{2}\right), \quad \Gamma_{d 2}=\Gamma_{d}-f_{1} \delta \Gamma_{d}
$$

$$
\frac{\partial \Gamma_{d 1}}{\partial x}=\frac{\partial \Gamma_{d}}{\partial x}-\delta \Gamma_{d} \frac{\partial f_{1}}{\partial x}+f_{2} \frac{\partial \delta \Gamma_{d}}{\partial x}, \quad \frac{\partial \Gamma_{d 2}}{\partial x}=\frac{\partial \Gamma_{d}}{\partial x}-\delta \Gamma_{d} \frac{\partial f_{1}}{\partial x}-f_{1} \frac{\partial \delta \Gamma_{d}}{\partial x}
$$

$$
\mathbf{R}_{\mathrm{o}}=R_{0} \hat{\mathrm{x}}^{2}, \quad R_{0} \equiv \delta: \mathbf{R}_{0}=\rho x_{1} x_{2} \delta w^{2}, \quad \rho k_{o}=\frac{1}{2} R_{o}
$$

$$
\mathbf{R}_{\mathrm{d}}=\frac{2}{3} \rho k_{d} \delta+\frac{2}{3} \Gamma_{d}\left(\delta-3 \hat{\mathrm{x}}^{2}\right),
$$

$$
\mathbf{R}_{\mathrm{d} 1}=\frac{2}{3} \rho k_{d} \delta+\frac{2}{3} \Gamma_{d 1}\left(\delta-3 \hat{\mathbf{x}}^{2}\right), \quad \mathbf{R}_{\mathrm{d} 2}=\frac{2}{3} \rho k_{d} \delta+\frac{2}{3} \Gamma_{d 2}\left(\delta-3 \hat{\mathbf{x}}^{2}\right),
$$

$$
\begin{aligned}
& \rho \phi_{d}=\mathbf{R}_{\mathbf{d}}: \hat{\mathrm{x}}^{2}\left(\frac{\partial u}{\partial x}-\delta w \frac{\partial x_{1}}{\partial x}\right)+\left(f_{1}-x_{1}\right) \mathbf{R}_{\mathbf{d} 1}: \hat{\mathbf{x}}^{2} \frac{\partial \delta w}{\partial x} \\
& =\left(\frac{2}{3} \rho k_{d}-\frac{4}{3} \Gamma_{d}\right)\left(\frac{\partial u}{\partial x}-\delta w \frac{\partial x_{1}}{\partial x}\right)+\left[\left(f_{1}-x_{1}\right)\left(\frac{2}{3} \rho k_{d}-\frac{4}{3} \Gamma_{d}\right)-\frac{4}{3} f_{1} f_{2} \delta \Gamma_{d}\right] \frac{\partial \delta w}{\partial x} \\
& =\left(\frac{2}{3} \rho k_{d}-\frac{4}{3} \Gamma_{d}\right)\left[\frac{\partial u}{\partial x}-\delta w \frac{\partial x_{1}}{\partial x}+\left(f_{1}-x_{1}-f_{1} f_{2} \frac{\delta \Gamma_{d}}{\rho k_{d} / 2-\Gamma_{d}}\right) \frac{\partial \delta w}{\partial x}\right] . \\
& \Pi_{o}=\frac{2}{3} \Pi_{\circ}\left(\delta-3 \hat{\mathbf{x}}^{2}\right), \quad \delta \Pi_{\circ}=\frac{2}{3} \delta \Pi_{\circ}\left(\delta-3 \hat{\mathrm{x}}^{2}\right), \\
& \Pi_{0}=\eta\left[\frac{\partial u}{\partial x}-\delta w \frac{\partial x_{1}}{\partial x}+\left(f_{1}-x_{1}+f_{1} f_{2} \frac{\delta \eta}{\eta}\right) \frac{\partial \delta w}{\partial x}\right] \\
& \delta \Pi_{\circ}=\delta \eta\left[\frac{\partial u}{\partial x}-\delta w \frac{\partial x_{1}}{\partial x}+\left(f_{2}-x_{1}+\frac{\eta}{\delta \eta}\right) \frac{\partial \delta w}{\partial x}\right], \\
& \Pi_{1}=\frac{2}{3} \Pi_{1}\left(\delta-3 \hat{\mathbf{x}}^{2}\right), \quad \Pi_{2}=\frac{2}{3} \Pi_{2}\left(\delta-3 \hat{\mathbf{x}}^{2}\right), \\
& \Pi_{1}=\left(\eta+f_{2} \delta \eta\right)\left(\frac{\partial u}{\partial x}-\delta w \frac{\partial x_{1}}{\partial x}+x_{2} \frac{\partial \delta w}{\partial x}\right), \\
& \Pi_{2}=\left(\eta-f_{1} \delta \eta\right)\left(\frac{\partial u}{\partial x}-\delta w \frac{\partial x_{1}}{\partial x}-x_{1} \frac{\partial \delta w}{\partial x}\right), \\
& \mathbf{Q}=\frac{2}{3} Q\left(\delta-3 \hat{\mathbf{x}}^{2}\right), \quad \delta \mathbf{Q}=\frac{2}{3} \delta Q\left(\delta-3 \hat{\mathbf{x}}^{2}\right), \\
& Q=\eta\left[-\delta w \frac{\partial x_{1}}{\partial x}+\left(f_{1}-x_{1}+f_{1} f_{2} \frac{\delta \eta}{\eta}\right) \frac{\partial \delta w}{\partial x}\right]=\Pi_{o}-\eta \frac{\partial u}{\partial x}, \\
& \delta Q=\delta \eta\left[-\delta w \frac{\partial x_{1}}{\partial x}+\left(f_{2}-x_{1}+\frac{\eta}{\delta \eta}\right) \frac{\partial \delta w}{\partial x}\right]=\delta \Pi_{\circ}-\delta \eta \frac{\partial u}{\partial x},
\end{aligned}
$$




$$
\begin{aligned}
& \Pi_{1}^{\prime}=\frac{2}{3} \Pi_{1}^{\prime}\left(\delta-3 \hat{\mathbf{x}}^{2}\right), \quad \Pi_{2}^{\prime}=\frac{2}{3} \Pi_{2}^{\prime}\left(\delta-3 \hat{\mathbf{x}}^{2}\right), \\
& \Pi_{1}^{\prime}=\left(\eta+f_{2} \delta \eta\right)\left(-\delta w \frac{\partial x_{1}}{\partial x}+x_{2} \frac{\partial \delta w}{\partial x}\right)=\Pi_{1}-\eta_{1} \frac{\partial u}{\partial x}, \\
& \Pi_{2}^{\prime}=\left(\eta-f_{1} \delta \eta\right)\left(-\delta w \frac{\partial x_{1}}{\partial x}-x_{1} \frac{\partial \delta w}{\partial x}\right)=\Pi_{2}-\eta_{2} \frac{\partial u}{\partial x} \\
& \Phi=\frac{2}{3} \Phi\left(\delta-3 \hat{\mathrm{x}}^{2}\right), \quad \delta \Phi=\frac{2}{3} \delta \Phi\left(\delta-3 \hat{\mathrm{x}}^{2}\right), \\
& \Phi=\Pi_{o}+\Gamma_{d}=\left(\eta+\eta_{d}\right)\left[\frac{\partial u}{\partial x}-\delta w \frac{\partial x_{1}}{\partial x}+\left(f_{1}-x_{1}+f_{1} f_{2} \frac{\delta \eta}{\eta+\eta_{d}}\right) \frac{\partial \delta w}{\partial x}\right], \\
& \delta \Phi=\delta \Pi_{\circ}+\delta \Gamma_{d}=\delta \eta\left[\frac{\partial u}{\partial x}-\delta w \frac{\partial x_{1}}{\partial x}+\left(f_{2}-x_{1}+\frac{\eta+\eta_{d}}{\delta \eta}\right) \frac{\partial \delta w}{\partial x}\right] \\
& \mathbf{P}=P \delta+\frac{2}{3} \Pi_{\circ}\left(\delta-3 \hat{\mathbf{x}}^{2}\right), \\
& \vec{s}_{\circ}=\left(\rho x_{1} w_{1} \theta_{1}+\rho x_{2} w_{2} \theta_{2}\right) \hat{\mathrm{x}}=\rho x_{1} x_{2} \delta w \delta \theta \hat{\mathrm{x}} \\
& \vec{s}_{d}=-C_{i d}\left(\frac{\partial i}{\partial T}\right)_{\rho} \vec{D}_{d x} \frac{\partial T}{\partial x} \\
& \vec{D}_{d x} \equiv \mathrm{D}_{\mathbf{d}} \cdot \hat{\mathbf{x}}=D_{d x} \hat{\mathbf{x}}, \quad D_{d x}=\omega^{-1}\left(\frac{2}{3} \rho k_{d}-\frac{4}{3} \Phi\right), \\
& \mathbf{D}_{\mathrm{d}}=\omega^{-1}\left(\mathbf{R}_{\mathrm{d}}+\mathbf{\Pi}_{\circ}\right)=\omega^{-1}\left[\frac{2}{3} \rho k_{d} \delta+\frac{2}{3} \Phi\left(\delta-3 \hat{\mathbf{x}}^{2}\right)\right] \text {, } \\
& \vec{q}=-\left(f_{1} \kappa_{1}+f_{2} \kappa_{2}\right) \frac{\partial T}{\partial x} \hat{\mathrm{x}}=-\kappa \frac{\partial T}{\partial x} \hat{\mathrm{x}}, \\
& \rho \epsilon_{o}=\mathbf{Q}: \hat{\mathbf{x}}^{2} \delta w \frac{\partial x_{1}}{\partial x}+\left(x_{1} \mathbf{Q}-f_{1} \boldsymbol{\Pi}_{1}^{\prime}\right): \hat{\mathbf{x}}^{2} \frac{\partial \delta w}{\partial x} \\
& =\frac{4}{3} Q\left[-\delta w \frac{\partial x_{1}}{\partial x}+\left(f_{1}-x_{1}+f_{1} f_{2} \frac{\delta Q}{Q}\right) \frac{\partial \delta w}{\partial x}\right] .
\end{aligned}
$$

Note that $\delta: \hat{\mathbf{x}}^{2}=1, f_{2}=1-f_{1}$, and $x_{2}=1-x_{1}$. For simplicity, we introduce $a_{1}=x_{1} x_{2}=$ $x_{1}\left(1-x_{1}\right), a_{2}=x_{2}-x_{1}=1-2 x_{1}$, and $a_{3}=f_{1}-x_{1}$. With these expressions, in the case of both pressure and temperature equilibrium, the one-dimensional evolution equations for the two-fluid system reduce to

$$
\begin{gathered}
\frac{\partial \rho}{\partial t}+u \frac{\partial \rho}{\partial x}+\rho \frac{\partial u}{\partial x}=0 \\
\rho \frac{\partial u}{\partial t}+\left(\frac{2}{3} k_{d}+a_{1} \delta w^{2}\right) \frac{\partial \rho}{\partial x}+\rho u \frac{\partial u}{\partial x}+\rho a_{2} \delta w^{2} \frac{\partial x_{1}}{\partial x}+2 \rho a_{1} \delta w \frac{\partial \delta w}{\partial x}+ \\
\frac{2}{3} \rho \frac{\partial k_{d}}{\partial x}-\frac{4}{3} \frac{\partial \Phi}{\partial x}+\frac{\partial P}{\partial x}=0 \\
\rho \frac{\partial x_{1}}{\partial t}+a_{1} \delta w \frac{\partial \rho}{\partial x}+\rho\left(u+a_{2} \delta w\right) \frac{\partial x_{1}}{\partial x}+\rho a_{1} \frac{\partial \delta w}{\partial x}=0
\end{gathered}
$$




$$
\begin{aligned}
& \rho x_{1} \frac{\partial u}{\partial t}+\rho a_{2} \delta w \frac{\partial x_{1}}{\partial t}+\rho a_{1} \frac{\partial \delta w}{\partial t}+\left[\frac{2}{3} f_{1} k_{d}+a_{1}\left(1-x_{1}\right) \delta w^{2}\right] \frac{\partial \rho}{\partial x}+ \\
& \rho x_{1}\left[u+\left(1-x_{1}\right) \delta w\right] \frac{\partial u}{\partial x}+\rho\left[a_{2} u+\left(1-x_{1}\right)\left(1-3 x_{1}\right) \delta w\right] \delta w \frac{\partial x_{1}}{\partial x}+\rho a_{1}\left[u+2\left(1-x_{1}\right) \delta w\right] \frac{\partial \delta w}{\partial x}+ \\
& \frac{2}{3} f_{1} \rho \frac{\partial k_{d}}{\partial x}-\frac{4}{3} f_{1}\left[\frac{\partial \Phi}{\partial x}+\left(1-f_{1}\right) \frac{\partial \delta \Phi}{\partial x}\right]+f_{1} \frac{\partial P}{\partial x}+ \\
& {\left[\frac{2}{3} \rho k_{d}-\frac{4}{3}\left(\Phi+\left(1-2 f_{1}\right) \delta \Phi\right)\right] \frac{\partial f_{1}}{\partial x}+\omega a_{1} \rho \delta w=0,} \\
& \rho \frac{\partial i}{\partial t}+a_{1} \delta \theta \delta w \frac{\partial \rho}{\partial x}+\left[P-\frac{4}{3}\left(\Pi_{\circ}+Q\right)\right] \frac{\partial u}{\partial x}+\left(a_{2} \rho \delta \theta+\frac{4}{3} Q-P\right) \delta w \frac{\partial x_{1}}{\partial x}+ \\
& {\left[a_{1} \rho \delta \theta+a_{3} P-\frac{4}{3} Q a_{3}-\frac{4}{3} \delta Q f_{1}\left(1-f_{1}\right)\right] \frac{\partial \delta w}{\partial x}+\rho u \frac{\partial i}{\partial x}+} \\
& \frac{\partial s_{d}}{\partial x}+\frac{\partial q}{\partial x}+P \delta w \frac{\partial f_{1}}{\partial x}+\rho a_{1} \delta w \frac{\partial \delta \theta}{\partial x}-\rho \epsilon_{d}=0, \\
& \rho \frac{\partial k_{d}}{\partial t}+k_{d} a_{3} \delta w \frac{\partial \rho}{\partial x}+\left(\frac{2}{3} \rho k_{d}-\frac{4}{3} \Gamma_{d}\right) \frac{\partial u}{\partial x}-\left(\frac{5}{3} \rho k_{d}-\frac{4}{3} \Gamma_{d}\right) \delta w \frac{\partial x_{1}}{\partial x}+ \\
& {\left[\frac{5}{3} \rho k_{d} a_{3}-\frac{4}{3} \Gamma_{d} a_{3}-\frac{4}{3} f_{1}\left(1-f_{1}\right) \delta \Gamma_{d}\right] \frac{\partial \delta w}{\partial x}+\rho\left(u+a_{3} \delta w\right) \frac{\partial k_{d}}{\partial x}+\frac{\partial J_{k}}{\partial x}+} \\
& \rho k_{d} \delta w \frac{\partial f_{1}}{\partial x}-\omega \rho a_{1} \delta w^{2}+\rho \epsilon_{d}=0 \\
& \rho \frac{\partial l}{\partial t}+a_{3} l \delta w \frac{\partial \rho}{\partial x}-C_{l o} \rho l \frac{\partial u}{\partial x}-\rho l \delta w \frac{\partial x_{1}}{\partial x}+a_{3} \rho l \frac{\partial \delta w}{\partial x}+\rho\left(u+a_{3} \delta w\right) \frac{\partial l}{\partial x}+ \\
& \frac{\partial J_{l}}{\partial x}+\rho l \delta w \frac{\partial f_{1}}{\partial x}-C_{l o} \rho \sqrt{k_{\circ}+k_{d}}=0 .
\end{aligned}
$$

The total viscous stress $\Phi$ and its variation $\delta \Phi$ are given by Eqs.(91) and (92), and the fluxes of internal energy, $s_{d} \hat{\mathbf{x}}$, heat, $q \hat{\mathrm{x}}$, turbulent kinetic energy, $J_{k} \hat{\mathbf{x}}$, and turbulent length scale, $J_{l} \hat{\mathbf{x}}$, satisfy the following auxiliary equations

$$
\begin{gathered}
s_{d}+D_{s} \frac{\partial T}{\partial x}=0, \quad D_{s}=C_{i d}\left(\frac{\partial i}{\partial T}\right)_{p} D_{d x} \\
q+D_{q} \frac{\partial T}{\partial x}=0, \quad D_{q}=\kappa \\
J_{k}+D_{k} \frac{\partial k_{d}}{\partial x}=0, \quad D_{k}=C_{k d} D_{d x} \\
J_{l}+D_{l} \frac{\partial l}{\partial x}=0, \quad D_{l}=C_{l d} D_{d x}
\end{gathered}
$$

Here the introduction of the fluxes is purely for the characteristic analysis of the system, that is, to avoid second derivatives of the dynamic quantities appearing in the equations. The $f_{j}, \delta \theta, P$, and $T$ will be determined from the equations of the state of the system. For ideal gases, the relationships among them are much simpler than for real fluids. 
It is clear that the one-dimensional evolution equations presented above in the case of pressure equilibrium along with the equations of state form a complete and general description of the system.

3.2 Ideal gases. For simplicity, we now apply the general one-dimensional evolution equations to a system with two ideal gases.

The equation of state for each ideal gas is described by

$$
P_{j}=\rho_{j} \theta_{j}\left(\gamma_{j}-1\right) / f_{j}, \quad \text { or } \quad P_{j}=\frac{N_{j}}{V_{j}} k_{B} T_{j}
$$

where $\gamma_{j}$ is the ratio of specific heats of the $j$-th material, $k_{B}$ is Boltzmann's constant, $N_{j} / V_{j}=\Gamma_{j} \rho_{j} / f_{j}, \Gamma_{j} \equiv N_{A v} / A_{j}, N_{A v}=6.02 \times 10^{23}$ is Avogadro's number, $V_{j} \equiv f_{j} V$ and $A_{j}$ respectively express the volume and mass number of the $j$-th material, and $V$ is the total volume of the system. In the situation of both pressure and temperature equilibrium, the volume fraction $\left(f_{j}\right)$ of the $j$-th material is determined directly from the equations of state

$$
f_{j}=\frac{\Gamma_{j} \rho_{j}}{\sum_{j^{\prime}} \Gamma_{j^{\prime}} \rho_{j^{\prime}}}
$$

For two materials $(j=1,2)$, this gives

$$
f_{j}=\frac{\Gamma_{j} \rho_{j}}{\Gamma_{1} \rho_{1}+\Gamma_{2} \rho_{2}}=\frac{\Gamma_{j} x_{j}}{\Gamma_{1} x_{1}+\Gamma_{2} x_{2}} .
$$

This leads to useful identities:

$$
\begin{aligned}
& \frac{\Gamma_{1} x_{1}}{f_{1}}=\frac{\Gamma_{2} x_{2}}{f_{2}} \text { or } \frac{\Gamma_{2}}{\Gamma_{1}}=\frac{f_{2} x_{1}}{x_{2} f_{1}}, \\
& \Gamma_{1}-\Gamma_{2}=\frac{\Gamma_{1}}{f_{1}}-\frac{\Gamma_{2}}{x_{1}}=\frac{\Gamma_{1}}{x_{2}}-\frac{\Gamma_{2}}{f_{2}} .
\end{aligned}
$$

With these expressions, we can write the following thermodynamical quantities and their derivatives explicitly,

$$
\begin{gathered}
P_{1}=P_{2}=P=\left(\Gamma_{1} x_{1}+\Gamma_{2} x_{2}\right) \rho k_{B} T \\
\theta_{j}=\frac{\Gamma_{j}}{\gamma_{j}-1} k_{B} T \\
i=x_{1} \theta_{1}+x_{2} \theta_{2}=\frac{P}{\rho(\gamma-1)}, \quad \frac{1}{\gamma-1}=\frac{f_{1}}{\gamma_{1}-1}+\frac{f_{2}}{\gamma_{2}-1}, \\
\delta \theta \equiv \theta_{1}-\theta_{2}=k_{B} T\left(\frac{\Gamma_{1}}{\gamma_{1}-1}-\frac{\Gamma_{2}}{\gamma_{2}-1}\right), \\
\frac{\partial f_{1}}{\partial t}=\frac{\Gamma_{1} \Gamma_{2}}{\left(\Gamma_{1} x_{1}+\Gamma_{2} x_{2}\right)^{2}} \frac{\partial x_{1}}{\partial t}=\frac{f_{1} f_{2}}{x_{1} x_{2}} \frac{\partial x_{1}}{\partial t} \\
\frac{\partial f_{1}}{\partial x}=\frac{\Gamma_{1} \Gamma_{2}}{\left(\Gamma_{1} x_{1}+\Gamma_{2} x_{2}\right)^{2}} \frac{\partial x_{1}}{\partial x}=\frac{f_{1} f_{2}}{x_{1} x_{2}} \frac{\partial x_{1}}{\partial x}
\end{gathered}
$$


THE CHARACTERISTIC ANALYSIS OF A HYBRID MULTIFLUID TURBULENT-MIX MODEL

$$
\begin{gathered}
-\frac{1}{(\gamma-1)^{2}} \frac{\partial \gamma}{\partial t}=\left(\frac{1}{\gamma_{1}-1}-\frac{1}{\gamma_{2}-1}\right) \frac{\partial f_{1}}{\partial t}, \\
-\frac{1}{(\gamma-1)^{2}} \frac{\partial \gamma}{\partial x}=\left(\frac{1}{\gamma_{1}-1}-\frac{1}{\gamma_{2}-1}\right) \frac{\partial f_{1}}{\partial x} \\
\frac{\partial P}{\partial x}=\left(\Gamma_{1}-\Gamma_{2}\right) \rho k_{B} T \frac{\partial x_{1}}{\partial x}+\frac{\Gamma_{1} x_{1}}{f_{1}} k_{B} T \frac{\partial \rho}{\partial x}+\frac{\Gamma_{1} x_{1}}{f_{1}} \rho k_{B} \frac{\partial T}{\partial x} \\
=\left(\frac{f_{1}}{x_{1}}-\frac{f_{2}}{x_{2}}\right) P \frac{\partial x_{1}}{\partial x}+\frac{P}{\rho} \frac{\partial \rho}{\partial x}+\frac{P}{T} \frac{\partial T}{\partial x}, \\
\frac{\partial i}{\partial t}=\frac{i \frac{\partial T}{T}+\delta \theta \frac{\partial x_{1}}{\partial t}, \quad \frac{\partial i}{\partial x}=\frac{i}{T} \frac{\partial T}{\partial x}+\delta \theta \frac{\partial x_{1}}{\partial x}}{} \\
\frac{\partial \delta \theta}{\partial t}=k_{B}\left(\frac{\Gamma_{1}}{\gamma_{1}-1}-\frac{\Gamma_{2}}{\gamma_{2}-1}\right) \frac{\partial T}{\partial t}, \\
\frac{\partial \delta \theta}{\partial x}=k_{B}\left(\frac{\Gamma_{1}}{\gamma_{1}-1}-\frac{\Gamma_{2}}{\gamma_{2}-1}\right) \frac{\partial T}{\partial x} .
\end{gathered}
$$

Here $\gamma$ is regarded as the average ratio of specific heats of the system (i.e., the total material). Substituting these into the general one-dimensional evolution equations presented in the previous subsection gives the equations for two ideal gases

$$
\begin{gathered}
\frac{\partial \rho}{\partial t}+u \frac{\partial \rho}{\partial x}+\rho \frac{\partial u}{\partial x}=0 \\
\rho \frac{\partial u}{\partial t}+\left(\frac{2}{3} k_{d}+a_{1} \delta w^{2}+\frac{P}{\rho}\right) \frac{\partial \rho}{\partial x}+\rho u \frac{\partial u}{\partial x}+\rho\left[a_{2} \delta w^{2}+\left(\Gamma_{1}-\Gamma_{2}\right) k_{B} T\right] \frac{\partial x_{1}}{\partial x}+ \\
2 \rho a_{1} \delta w \frac{\partial \delta w}{\partial x}+\frac{P}{T} \frac{\partial T}{\partial x}+\frac{2}{3} \rho \frac{\partial k_{d}}{\partial x}-\frac{4}{3} \frac{\partial \Phi}{\partial x}=0 \\
\rho \frac{\partial x_{1}}{\partial t}+a_{1} \delta w \frac{\partial \rho}{\partial x}+\rho\left(u+a_{2} \delta w\right) \frac{\partial x_{1}}{\partial x}+\rho a_{1} \frac{\partial \delta w}{\partial x}=0, \\
\rho x_{1} \frac{\partial u}{\partial t}+\rho a_{2} \delta w \frac{\partial x_{1}}{\partial t}+\rho a_{1} \frac{\partial \delta w}{\partial t}+\left[\frac{2}{3} f_{1} k_{d}+a_{1}\left(1-x_{1}\right) \delta w^{2}+f_{1} \frac{P}{\rho}\right] \frac{\partial \rho}{\partial x}+ \\
\left.\frac{2}{3} \rho k_{d} \frac{f_{1} f_{2}}{x_{1} x_{2}}-\frac{4}{3}\left(\Phi+\left(1-2 f_{1}\right) \delta \Phi\right) \frac{f_{1} f_{2}}{x_{1} x_{2}}\right] \frac{\partial x_{1}}{\partial x}+\rho a_{1}\left[u+2\left(1-x_{1}\right) \delta w\right] \frac{\partial \delta w}{\partial x}+ \\
f_{1} \frac{P}{T} \frac{\partial T}{\partial x}+\frac{2}{3} f_{1} \rho \frac{\partial k_{d}}{\partial x}-\frac{4}{3} f_{1}\left[\frac{\partial \Phi}{\partial x}+\left(1-f_{1}\right) \frac{\partial \delta \Phi}{\partial x}\right]+w a_{1} \rho \delta w=0
\end{gathered}
$$




$$
\begin{aligned}
& \rho \delta \theta \frac{\partial x_{1}}{\partial t}+\rho \frac{i}{T} \frac{\partial T}{\partial t}+a_{1} \delta \theta \delta w \frac{\partial \rho}{\partial x}+\left[P-\frac{4}{3}\left(\Pi_{o}+Q\right)\right] \frac{\partial u}{\partial x}+ \\
& {\left[a_{2} \rho \delta \theta \delta w+\left(\frac{4}{3} Q-P\right) \delta w+\rho u \delta \theta\right] \frac{\partial x_{1}}{\partial x}+\left(a_{1} \rho \delta \theta+a_{3} P-\frac{4}{3} Q a_{3}-\frac{4}{3} \delta Q f_{1} f_{2}\right) \frac{\partial \delta w}{\partial x}+} \\
& \frac{\rho}{T}\left(i u+a_{1} \delta \theta \delta w\right) \frac{\partial T}{\partial x}+\frac{\partial s_{d}}{\partial x}+\frac{\partial q}{\partial x}-\rho \epsilon_{d}=0, \\
& \rho \frac{\partial k_{d}}{\partial t}+k_{d} a_{3} \delta w \frac{\partial \rho}{\partial x}+\left(\frac{2}{3} \rho k_{d}-\frac{4}{3} \Gamma_{d}\right) \frac{\partial u}{\partial x}-\left[\left(\frac{5}{3}-\frac{f_{1} f_{2}}{x_{1} x_{2}}\right) \rho k_{d}-\frac{4}{3} \Gamma_{d}\right] \delta w \frac{\partial x_{1}}{\partial x}+ \\
& \left(\frac{5}{3} \rho k_{d} a_{3}-\frac{4}{3} \Gamma_{d} a_{3}-\frac{4}{3} f_{1} f_{2} \delta \Gamma_{d}\right) \frac{\partial \delta w}{\partial x}+\rho\left(u+a_{3} \delta w\right) \frac{\partial k_{d}}{\partial x}+ \\
& \frac{\partial J_{k}}{\partial x}-\omega \rho a_{1} \delta w^{2}+\rho \epsilon_{d}=0 \\
& \rho \frac{\partial l}{\partial t}+a_{3} l \delta w \frac{\partial \rho}{\partial x}-C_{l o} \rho l \frac{\partial u}{\partial x}-\rho l \delta w\left(1-\frac{f_{1} f_{2}}{x_{1} x_{2}}\right) \frac{\partial x_{1}}{\partial x}+a_{3} \rho l \frac{\partial \delta w}{\partial x}+\rho\left(u+a_{3} \delta w\right) \frac{\partial l}{\partial x}+ \\
& \frac{\partial J_{l}}{\partial x}-C_{l o} \rho \sqrt{k_{o}+k_{d}}=0 . \\
& \Phi-\left(\eta+\eta_{d}\right)\left[\frac{\partial u}{\partial x}-\delta w \frac{\partial x_{1}}{\partial x}+\left(a_{3}+f_{1} f_{2} \frac{\delta \eta}{\eta+\eta_{d}}\right) \frac{\partial \delta w}{\partial x}\right]=0 \\
& \delta \Phi-\delta \eta\left[\frac{\partial u}{\partial x}-\delta w \frac{\partial x_{1}}{\partial x}+\left(1-f_{1}-x_{1}+\frac{\eta+\eta_{d}}{\delta \eta}\right) \frac{\partial \delta w}{\partial x}\right]=0 \\
& s_{d}+D_{s} \frac{\partial T}{\partial x}=0, \quad D_{s}=C_{i d}\left(\frac{\partial i}{\partial T}\right)_{\rho} D_{d x} \\
& q+D_{q} \frac{\partial T}{\partial x}=0, \quad D_{q}=\kappa \\
& J_{k}+D_{k} \frac{\partial k_{d}}{\partial x}=0, \quad D_{k}=C_{k d} D_{d x} \\
& J_{l}+D_{l} \frac{\partial l}{\partial x}=0, \quad D_{l}=C_{l d} D_{d x} .
\end{aligned}
$$

We have expressed the dynamical variables $i, \delta \theta$, and $P$ as functions of mass fraction $x_{1}$, mass density $\rho$, and temperature $T$ by using the equations of state. For convenience, we introduce the following parameters

$$
\begin{gathered}
a_{4} \equiv 1-\frac{f_{1} f_{2}}{x_{1} x_{2}} \\
b_{1} \equiv \frac{P}{\rho}=\frac{\Gamma_{1} x_{1} k_{B} T}{f_{1}}, \quad b_{2} \equiv\left(\Gamma_{1}-\Gamma_{2}\right) k_{B} T \\
c_{1} \equiv \frac{2}{3} k_{d}-\frac{4}{3 \rho} \Gamma_{d}, \quad c_{2} \equiv\left[\frac{2}{3} k_{d}-\frac{4}{3 \rho}\left(\Phi+\left(1-2 f_{1}\right) \delta \Phi\right)\right] \frac{f_{1} f_{2}}{x_{1} x_{2}} .
\end{gathered}
$$


Moreover, in order to do the characteristic analysis of the system in the next section, we prefer to eliminate $\frac{\partial u}{\partial t}$ and $\frac{\partial x_{1}}{\partial t}$ from Eqs.(130) and (131) using their evolution equations. Thus the above equations for ideal gases are finally simplified into

$$
\begin{gathered}
\frac{\partial \rho}{\partial t}+u \frac{\partial \rho}{\partial x}+\rho \frac{\partial u}{\partial x}=0 \\
\frac{\partial u}{\partial t}+\left(\frac{2}{3} k_{d}+a_{1} \delta w^{2}+b_{1}\right) \frac{1}{\rho} \frac{\partial \rho}{\partial x}+u \frac{\partial u}{\partial x}+\left(a_{2} \delta w^{2}+b_{2}\right) \frac{\partial x_{1}}{\partial x}+2 a_{1} \delta w \frac{\partial \delta w}{\partial x}+ \\
\frac{b_{1}}{T} \frac{\partial T}{\partial x}+\frac{2}{3} \frac{\partial k_{d}}{\partial x}-\frac{4}{3 \rho} \frac{\partial \Phi}{\partial x}=0 \\
\frac{\partial x_{1}}{\partial t}+\frac{a_{1} \delta w}{\rho} \frac{\partial \rho}{\partial x}+\left(u+a_{2} \delta w\right) \frac{\partial x_{1}}{\partial x}+a_{1} \frac{\partial \delta w}{\partial x}=0
\end{gathered}
$$

$$
\begin{gathered}
\frac{\partial \delta w}{\partial t}+\left(\frac{2}{3} \frac{a_{3}}{a_{1}} k_{d}+\frac{a_{3}}{a_{1}} b_{1}\right) \frac{1}{\rho} \frac{\partial \rho}{\partial x}+\delta w \frac{\partial u}{\partial x}+\left(-\delta w^{2}+\frac{a_{3}}{a_{1}} b_{2}+\frac{c_{2}}{a_{1}}\right) \frac{\partial x_{1}}{\partial x}+\left(u+a_{2} \delta w\right) \frac{\partial \delta w}{\partial x}+ \\
\frac{a_{3}}{a_{1}} \frac{b_{1}}{T} \frac{\partial T}{\partial x}-\frac{2}{3} \frac{a_{3}}{a_{1}} \frac{\partial k_{d}}{\partial x}-\frac{4}{3 \rho} \frac{a_{3}}{a_{1}} \frac{\partial \Phi}{\partial x}+\frac{4}{3 \rho a_{1}} f_{1} f_{2} \frac{\partial \delta \Phi}{\partial x}+\omega \delta w=0, \\
\frac{\partial T}{\partial t}+\frac{(\gamma-1) T}{b_{1} \rho}\left[P-\frac{4}{3}\left(\Pi_{\circ}+Q\right)\right] \frac{\partial u}{\partial x}+\frac{(\gamma-1) T}{b_{1}}\left(\frac{4}{3 \rho} Q-a_{4} b_{1}\right) \delta w \frac{\partial x_{1}}{\partial x}+ \\
{\left[a_{3}\left(P-\frac{4}{3} Q\right)+\frac{4}{3} \delta Q f_{1} f_{2}\right] \frac{(\gamma-1) T}{b_{1} \rho} \frac{\partial \delta w}{\partial x}+\left(u+\frac{(\gamma-1) \delta \theta}{b_{1}} a_{1} \delta w\right) \frac{\partial T}{\partial x}+} \\
\frac{(\gamma-1) T}{b_{1} \rho} \frac{\partial}{\partial x}\left(s_{d}+q\right)-\frac{(\gamma-1) T}{b_{1}} \epsilon_{d}=0
\end{gathered}
$$

$$
\begin{gathered}
\frac{\partial k_{d}}{\partial t}+\frac{k_{d} a_{3} \delta w}{\rho} \frac{\partial \rho}{\partial x}+c_{1} \frac{\partial u}{\partial x}-\left(c_{1}+a_{4} k_{d}\right) \delta w \frac{\partial x_{1}}{\partial x}+\left[a_{3}\left(c_{1}+k_{d}\right)-\frac{4}{3 \rho} f_{1} f_{2} \delta \Gamma_{d}\right] \frac{\partial \delta w}{\partial x}+ \\
\left(u+a_{3} \delta w\right) \frac{\partial k_{d}}{\partial x}+\frac{1}{\rho} \frac{\partial J_{k}}{\partial x}-\omega a_{1} \delta w^{2}+\epsilon_{d}=0
\end{gathered}
$$$$
\frac{\partial l}{\partial t}+\frac{a_{3} l \delta w}{\rho} \frac{\partial \rho}{\partial x}-C_{l o} l \frac{\partial u}{\partial x}-a_{4} l \delta w \frac{\partial x_{1}}{\partial x}+a_{3} l \frac{\partial \delta w}{\partial x}+\left(u+a_{3} \delta w\right) \frac{\partial l}{\partial x}+
$$$$
\frac{1}{\rho} \frac{\partial J_{l}}{\partial x}-C_{l o} \sqrt{k_{o}+k_{d}}=0 \text {. }
$$$$
\Phi-\eta^{*}\left[\frac{\partial u}{\partial x}-\delta w \frac{\partial x_{1}}{\partial x}+\left(a_{3}+f_{1} f_{2} \frac{\delta \eta}{\eta^{*}}\right) \frac{\partial \delta w}{\partial x}\right]=0, \quad \eta^{*}=\eta+\eta_{d}
$$$$
\delta \Phi-\delta \eta\left[\frac{\partial u}{\partial x}-\delta w \frac{\partial x_{1}}{\partial x}+\left(a_{2}-a_{3}+\frac{\eta^{*}}{\delta \eta}\right) \frac{\partial \delta w}{\partial x}\right]=0
$$$$
S+D_{T} \frac{\partial T}{\partial x}=0, \quad S \equiv s_{d}+q, \quad D_{T} \equiv D_{s}+D_{q}=C_{i d}\left(\frac{\partial i}{\partial T}\right)_{\rho} D_{d x}+\kappa
$$ 


$$
\begin{gathered}
J_{k}+D_{k} \frac{\partial k_{d}}{\partial x}=0, \quad D_{k}=C_{k d} D_{d x} \\
J_{l}+D_{l} \frac{\partial l}{\partial x}=0, \quad D_{l}=C_{l d} D_{d x}
\end{gathered}
$$

Here we have introduced the total viscosity of the system, $\eta^{*}=\eta+\eta_{d}$, i.e., the sum of the real and turbulent viscosities. It is apparent that as long as one of the viscosities ( $\eta$ or $\left.\eta_{d}\right)$ does not vanish, $\eta^{*}$ will remain non-zero, so will $\Phi$ and $\delta \Phi$.

Manifestly, now the above set of equations can be cast into a matrix form as follows:

$$
\mathcal{A} \frac{\partial \mathcal{U}}{\partial t}+\mathcal{B} \frac{\partial \mathcal{U}}{\partial x}+\mathcal{C}=0
$$

where $\mathcal{A}$ and $\mathcal{B}$ are square matrices consisting of the coefficients of the derivatives of the dynamical variables in the equations. $C$ is a "source" column vector which contains the non-derivative terms in the equations. $U$ is a column vector whose components are the dynamical variables. The transpose of $\mathcal{U}$ is given by

$$
\mathcal{U}^{T} \equiv\left(\rho, u, x_{1}, \delta w, T, k_{d}, l, \Phi, \delta \Phi, S, J_{k}, J_{l}\right)
$$

We shall see that this expression is helpful throughout the characteristic analysis.

\section{Characteristics}

Now we consider the one-dimensitional two-fluid ideal-gas system discussed in the last section as an example.

It is well known that the condition of compatibility for the linear homogeneneous system of Eq.(153) to have non-trivial solutions is determined by the characteristic values $\lambda$ satisfying the equation

$$
|\mathcal{B}-\lambda \mathcal{A}|=0
$$

where $|\mathcal{M}|$ expresses the determinant of matrix $\mathcal{M}$. Note that the source vector $\mathcal{C}$ is by definition not included in the characteristic analysis. Referring to Eq.(153), $\mathcal{A}$ and $\mathcal{B}$ are $12 \times 12$ matrices that have the form

$$
\mathcal{A}=\left(a_{i} \delta_{i j}\right), \quad \mathcal{B}=\left(b_{i j}\right)
$$


where $a_{i}=1$, for $i=1,2, \ldots, 7 ; a_{i}=0$ for $i=8,9, \ldots, 12 ; \delta_{i j}$ is the Kronecker symbol;

$$
\begin{aligned}
& b_{11}=u, \quad b_{12}=\rho, \quad b_{1 i}=0, i=2,3, \ldots, 12 ; \\
& b_{21}=\left(\frac{2}{3} k_{d}+a_{1} \delta w^{2}+b_{1}\right) / \rho, \quad b_{22}=u, \quad b_{23}=a_{2} \delta w^{2}+b_{2}, \quad b_{24}=2 a_{1} \delta w, \\
& b_{25}=\frac{b_{1}}{T}, \quad b_{26}=\frac{2}{3}, \quad b_{27}=0, \quad b_{28}=-\frac{4}{3 \rho}, \quad b_{2 i}=0, i=9,10,11,12 ; \\
& b_{31}=a_{1} \delta w / \rho, \quad b_{32}=0, \quad b_{33}=u+a_{2} \delta w, \quad b_{34}=a_{1}, \quad b_{3 i}=0, i=5,6, \ldots, 12 \text {; } \\
& b_{41}=\left(\frac{2}{3} k_{d}+b_{1}\right) \frac{a_{3}}{a_{1} \rho}, \quad b_{42}=\delta w, \quad b_{43}=-\delta w^{2}+\frac{a_{3}}{a_{1}} b_{2}+\frac{c_{2}}{a_{1}}, \quad b_{44}=u+a_{2} \delta w, \\
& b_{45}=\frac{a_{3} b_{1}}{a_{1} T}, \quad b_{46}=\frac{2 a_{3}}{3 a_{1}}, \quad b_{47}=0, \quad b_{48}=-\frac{4}{3 \rho} \frac{a_{3}}{a_{1}}, \\
& b_{49}=-\frac{4}{3 \rho} \frac{f_{1} f_{2}}{a_{1}}, \quad b_{4 i}=0, i=10,11,12 \text {; } \\
& b_{51}=0, \quad b_{52}=\frac{(\gamma-1) T}{b_{1} \rho}\left[P-\frac{4}{3}\left(\Pi_{\circ}+Q\right)\right], \quad b_{53}=\frac{(\gamma-1) T}{b_{1} \rho}\left(\frac{4}{3} Q-a_{4} P\right) \delta w \\
& b_{54}=\frac{\gamma-1}{b_{1} \rho}\left[a_{3}\left(P-\frac{4}{3} \Pi_{0}\right)+\frac{4}{3} \delta Q f_{1} f_{2}\right], \quad b_{55}=u+\frac{(\gamma-1) \delta \theta}{b_{1}} a_{1} \delta w, \\
& b_{5 i}=0, i=6,7,8,9,11,12, \quad b_{510}=\frac{(\gamma-1) T}{b_{1} \rho} \\
& b_{61}=\frac{a_{3} k_{d} \delta w}{\rho}, \quad b_{62}=c_{1}, \quad b_{63}=-\left(c_{1}+a_{4} k_{d}\right) \delta w, \quad b_{64}=\left(c_{1}+k_{d}\right) a_{3}-\frac{4}{3 \rho} f_{1} f_{2} \delta \Gamma_{d_{3}} \\
& b_{65}=0, \quad b_{66}=u+a_{3} \delta w, \quad b_{6 i}=0, i=7,8,9,10,12, \quad b_{611}=\frac{1}{\rho} ; \\
& b_{71}=\frac{a_{3} l \delta w}{\rho}, \quad b_{72}=-C_{l o} l, \quad b_{73}=-a_{4} l \delta w, \quad b_{74}=a_{3} l, \quad b_{75}=b_{76}=0, \\
& b_{77}=u+a_{3} \delta w, \quad b_{7 i}=0, i=8,9,10,11, \quad b_{712}=1 / \rho \\
& b_{81}=0, \quad b_{82}=-\eta^{*}, \quad b_{83}=\eta^{*} \delta w, \quad b_{84}=-\eta^{*}\left(a_{3}+f_{1} f_{2} \frac{\delta \eta}{\eta^{*}}\right), \\
& b_{8 i}=0, i=5,6, \ldots, 12 \text {; } \\
& b_{91}=0, \quad b_{92}=-\delta \eta, \quad b_{93}=\delta w \delta \eta, \quad b_{94}=-\left(a_{2}-a_{3}\right) \delta \eta-\eta^{*} \text {, } \\
& b_{9 i}=0, i=5,6, \ldots, 12 \text {; } \\
& b_{10 i}=0, i \neq 5, \quad b_{105}=D_{T} \text {; } \\
& b_{11 i}=0, i \neq 6, \quad b_{116}=D_{k} \text {; } \\
& b_{12 i}=0, i \neq 7, \quad b_{127}=D_{l} \text {. }
\end{aligned}
$$

The source vector $\mathcal{C}$ is given by

$$
\mathfrak{C}^{T} \equiv\left(0,0,0, \omega \delta w,-\frac{(\gamma-1) T}{b_{1}} \epsilon_{d},-\omega a_{1} \delta w^{2}+\epsilon_{d},-C_{l o} \sqrt{k_{\circ}+k_{d}}, \Phi, \delta \Phi, S, J_{k}, J_{l}\right)
$$


Thus, the characteristic equation (155) can be expressed as

$\left.\begin{array}{cccccccccccc}b_{11}-\lambda & b_{12} & 0 & 0 & 0 & 0 & 0 & 0 & 0 & 0 & 0 & 0 \\ b_{21} & b_{22}-\lambda & b_{23} & b_{24} & b_{25} & b_{26} & 0 & b_{28} & 0 & 0 & 0 & 0 \\ b_{31} & 0 & b_{33}-\lambda & b_{34} & 0 & 0 & 0 & 0 & 0 & 0 & 0 & 0 \\ b_{41} & b_{42} & b_{43} & b_{44}-\lambda & b_{45} & b_{46} & 0 & b_{48} & b_{49} & 0 & 0 & 0 \\ b_{51} & b_{52} & b_{53} & b_{54} & b_{55}-\lambda & 0 & 0 & 0 & 0 & b_{510} & 0 & 0 \\ b_{61} & b_{62} & b_{63} & b_{64} & 0 & b_{66}-\lambda & 0 & 0 & 0 & 0 & b_{611} & 0 \\ b_{71} & b_{72} & b_{73} & b_{74} & 0 & 0 & b_{77}-\lambda & 0 & 0 & 0 & 0 & b_{712} \\ 0 & b_{82} & b_{83} & b_{84} & 0 & 0 & 0 & 0 & 0 & 0 & 0 & 0 \\ 0 & b_{92} & b_{93} & b_{94} & 0 & 0 & 0 & 0 & 0 & 0 & 0 & 0 \\ 0 & 0 & 0 & 0 & D_{T} & 0 & 0 & 0 & 0 & 0 & 0 & 0 \\ 0 & 0 & 0 & 0 & 0 & D_{k} & 0 & 0 & 0 & 0 & 0 & 0 \\ 0 & 0 & 0 & 0 & 0 & 0 & D_{l} & 0 & 0 & 0 & 0 & 0\end{array}\right)$

In the remainder of this section, we will analyze the characteristics, $\lambda$, of the model by solving this equation under various physical situations. In order to see the individual effects of the dissipations on the characteristics of the model, we divide our analysis into two classes: A. in the situations with viscosity and with or without various diffusions; $B$. in the situations without viscosity and with or without various diffusions.

\section{A. Non-zero viscosity $\left(\eta^{*} \neq 0\right)$.}

Case 1 Two-phase flow with all diffusions $\left(D_{T} \neq 0, D_{k} \neq 0, D_{l} \neq 0\right)$. In this case, the characteristic equation is

$$
b_{28} b_{49} b_{510} b_{611} b_{712} D_{T} D_{k} D_{l}\left|\begin{array}{cccc}
b_{11}-\lambda & b_{12} & 0 & 0 \\
b_{31} & 0 & b_{33}-\lambda & b_{34} \\
0 & b_{82} & b_{83} & b_{84} \\
0 & b_{92} & b_{93} & b_{94}
\end{array}\right|=0
$$

i.e.,

$$
\frac{16}{9} \frac{1}{\rho^{5}} \frac{f_{1} f_{2}}{a_{1}} \frac{(\gamma-1) T}{b_{1}} D_{T} D_{k} D_{l}\left|\begin{array}{cccc}
b_{11}-\lambda & b_{12} & 0 & 0 \\
b_{31} & 0 & b_{33}-\lambda & b_{34} \\
0 & b_{82} & b_{83} & b_{84} \\
0 & b_{92} & b_{93} & b_{94}
\end{array}\right|=0 .
$$

For non-zero $D_{T}, D_{k}$, and $D_{l}$, this equation requires

$$
\left|\begin{array}{cccc}
b_{11}-\lambda & b_{12} & 0 & 0 \\
b_{31} & 0 & b_{33}-\lambda & b_{34} \\
0 & b_{82} & b_{83} & b_{84} \\
0 & b_{92} & b_{93} & b_{94}
\end{array}\right|=0
$$


Utilizing the properties of matrices, do (column 2) $\times \frac{b_{11}-\lambda}{-b_{12}}+($ Column 1$)$, then this equation is simplified into

$$
b_{12}\left|\begin{array}{ccc}
b_{31} & b_{33}-\lambda & b_{34} \\
-b_{82}\left(b_{11}-\lambda\right) / b_{12} & b_{83} & b_{84} \\
-b_{92}\left(b_{11}-\lambda\right) / b_{12} & b_{93} & b_{94}
\end{array}\right|=0
$$

Since $b_{12}=\rho \neq 0$, we must have

$$
\left|\begin{array}{ccc}
b_{31} & b_{33}-\lambda & b_{34} \\
-b_{82}\left(b_{11}-\lambda\right) / b_{12} & b_{83} & b_{84} \\
-b_{92}\left(b_{11}-\lambda\right) / b_{12} & b_{93} & b_{94}
\end{array}\right|=0 .
$$

Expand this equation and note that

$$
b_{83} b_{94}-b_{93} b_{84}=\delta w\left(b_{92} b_{84}-b_{82} b_{94}\right) \text { and } b_{82} b_{93}=b_{83} b_{92}
$$

for all values of $\eta$ and $\eta_{d}$, we then further have

$$
\left[b_{31} \delta w-\left(b_{33}-\lambda\right)\left(b_{11}-\lambda\right) \frac{1}{b_{12}}\right]\left(b_{82} b_{94}-b_{92} b_{84}\right)=0 .
$$

Substituing the $b_{i j}$ s into this equation gives

$$
\frac{1}{\rho}\left[a_{1} \delta w^{2}-\left(u+a_{2} \delta w-\lambda\right)(u-\lambda)\right]\left[\eta^{* 2}+\left(f_{2}-f_{1}\right) \eta^{*} \delta \eta-f_{1} f_{2} \delta \eta^{2}\right]=0 .
$$

For non-zero $\eta^{*}$, the second bracketed expression does not vanish in general, so this leads to

$$
a_{1} \delta w^{2}-\left(u+a_{2} \delta w-\lambda\right)(u-\lambda)=0,
$$

and two real roots $\lambda$ are obtained:

$$
\begin{aligned}
& \lambda_{1}=u+x_{2} \delta w=u+w_{1}, \\
& \lambda_{2}=u-x_{1} \delta w=u+w_{2} .
\end{aligned}
$$

Therefore in the presence of all dissipations, the new hybrid turbulent mix model, like other two-phase flow models, has all real characteristics.

Case 2 Two-phase flow without temperature diffusion $\left(D_{T}=0, D_{k} \neq 0, D_{l} \neq 0\right)$. In this case, the characteristic equation is

$$
b_{28} b_{49} b_{611} b_{712} D_{k} D_{l}\left(b_{55}-\lambda\right)\left|\begin{array}{cccc}
b_{11}-\lambda & b_{12} & 0 & 0 \\
b_{31} & 0 & b_{33}-\lambda & b_{34} \\
0 & b_{82} & b_{83} & b_{84} \\
0 & b_{92} & b_{93} & b_{94}
\end{array}\right|=0
$$

i.e.,

$$
\frac{16}{9} \frac{1}{\rho^{4}} \frac{f_{1} f_{2}}{a_{1}} D_{k} D_{l}\left(b_{55}-\lambda\right)\left|\begin{array}{cccc}
b_{11}-\lambda & b_{12} & 0 & 0 \\
b_{31} & 0 & b_{33}-\lambda & b_{34} \\
0 & b_{82} & b_{83} & b_{84} \\
0 & b_{92} & b_{93} & b_{94}
\end{array}\right|=0
$$


For non-zero $D_{k}$ and $D_{l}$, three real roots $\lambda$ are obtained:

$$
\begin{gathered}
\lambda_{1}=u+x_{2} \delta w=u+w_{1}, \\
\lambda_{2}=u-x_{1} \delta w=u+w_{2}, \\
\lambda_{3}=b_{55}=u+\frac{(\gamma-1) \delta \theta}{b_{1}} a_{1} \delta w .
\end{gathered}
$$

Again the model has all real characteristics.

Case 3 Two-phase flow without turbulent kinetic energy diffusion $\left(D_{k}=0, D_{T} \neq 0, D_{l} \neq 0\right)$. In this case, the characteristic equation is

$$
b_{28} b_{49} b_{510} b_{712} D_{T} D_{l}\left(b_{66}-\lambda\right)\left|\begin{array}{cccc}
b_{11}-\lambda & b_{12} & 0 & 0 \\
b_{31} & 0 & b_{33}-\lambda & b_{34} \\
0 & b_{82} & b_{83} & b_{84} \\
0 & b_{92} & b_{93} & b_{94}
\end{array}\right|=0
$$

i.e.,

$$
\frac{16}{9} \frac{1}{\rho^{4}} \frac{f_{1} f_{2}}{a_{1}} \frac{(\gamma-1) T}{b_{1}} D_{T} D_{l}\left(b_{66}-\lambda\right)\left|\begin{array}{cccc}
b_{11}-\lambda & b_{12} & 0 & 0 \\
b_{31} & 0 & b_{33}-\lambda & b_{34} \\
0 & b_{82} & b_{83} & b_{84} \\
0 & b_{92} & b_{93} & b_{94}
\end{array}\right|=0 .
$$

For non-zero $D_{T}$ and $D_{l}$, three real roots $\lambda$ are obtained:

$$
\begin{gathered}
\lambda_{1}=u+x_{2} \delta w=u+w_{1}, \\
\lambda_{2}=u-x_{1} \delta w=u+w_{2} \\
\lambda_{3}=b_{66}=u+\left(f_{1}-x_{1}\right) \delta w=u+w .
\end{gathered}
$$

Case \& Two-phase flow without turbulent length scale diffusion $\left(D_{l}=0, D_{T} \neq 0, D_{k} \neq 0\right)$. In this case, the characteristic is

$$
b_{28} b_{49} b_{510} b_{611} D_{T} D_{k}\left(b_{77}-\lambda\right)\left|\begin{array}{cccc}
b_{11}-\lambda & b_{12} & 0 & 0 \\
b_{31} & 0 & b_{33}-\lambda & b_{34} \\
0 & b_{82} & b_{83} & b_{84} \\
0 & b_{92} & b_{93} & b_{94}
\end{array}\right|=0
$$

i.e.,

$$
\frac{16}{9} \frac{1}{\rho^{4}} \frac{f_{1} f_{2}}{a_{1}} \frac{(\gamma-1) T}{b_{1}} D_{T} D_{k}\left(b_{77}-\lambda\right)\left|\begin{array}{cccc}
b_{11}-\lambda & b_{12} & 0 & 0 \\
b_{31} & 0 & b_{33}-\lambda & b_{34} \\
0 & b_{82} & b_{83} & b_{84} \\
0 & b_{92} & b_{93} & b_{94}
\end{array}\right|=0 .
$$

It is evident that the roots $\lambda$ are the same as in Case 3:

$$
\begin{gathered}
\lambda_{1}=u+x_{2} \delta w=u+w_{1} \\
\lambda_{2}=u-x_{1} \delta w=u+w_{2} \\
\lambda_{3}=b_{77}=u+\left(f_{1}-x_{1}\right) \delta w=u+w
\end{gathered}
$$


Case 5 Two-phase flow without turbulent diffusions $\left(D_{k}=0=D_{l}, D_{T} \neq 0\right)$. In this case, the characteristic equation is

$$
b_{28} b_{49} b_{510} D_{T}\left(b_{66}-\lambda\right)\left(b_{77}-\lambda\right)\left|\begin{array}{cccc}
b_{11}-\lambda & b_{12} & 0 & 0 \\
b_{31} & 0 & b_{33}-\lambda & b_{34} \\
0 & b_{82} & b_{83} & b_{84} \\
0 & b_{92} & b_{93} & b_{94}
\end{array}\right|=0
$$

i.e.,

$$
\frac{16}{9} \frac{1}{\rho^{3}} \frac{f_{1} f_{2}}{a_{1}} \frac{(\gamma-1) T}{b_{1}}\left(b_{66}-\lambda\right)\left(b_{77}-\lambda\right)\left|\begin{array}{cccc}
b_{11}-\lambda & b_{12} & 0 & 0 \\
b_{31} & 0 & b_{33}-\lambda & b_{34} \\
0 & b_{82} & b_{83} & b_{84} \\
0 & b_{92} & b_{93} & b_{94}
\end{array}\right|=0
$$

Four real roots $\lambda$ are obtained:

$$
\begin{aligned}
\lambda_{1} & =u+x_{2} \delta w=u+w_{1}, \\
\lambda_{2} & =u-x_{1} \delta w=u+w_{2}, \\
\lambda_{3}=b_{66} & =u+\left(f_{1}-x_{1}\right) \delta w=u+w \\
\lambda_{4}=b_{77} & =u+\left(f_{1}-x_{1}\right) \delta w=u+w .
\end{aligned}
$$

Case 6 Two-phase flow without temperature and turbulent kinetic energy diffusions $\left(D_{T}=\right.$ $0=D_{k}, D_{l} \neq 0$ ). In this case, the characteristic equation is

$$
b_{28} b_{49} b_{712} D_{l}\left(b_{55}-\lambda\right)\left(b_{66}-\lambda\right)\left|\begin{array}{cccc}
b_{11}-\lambda & b_{12} & 0 & 0 \\
b_{31} & 0 & b_{33}-\lambda & b_{34} \\
0 & b_{82} & b_{83} & b_{84} \\
0 & b_{92} & b_{93} & b_{94}
\end{array}\right|=0
$$

i.e.,

$$
\frac{16}{9} \frac{1}{\rho^{3}} \frac{f_{1} f_{2}}{a_{1}} D_{l}\left(b_{55}-\lambda\right)\left(b_{66}-\lambda\right)\left|\begin{array}{cccc}
b_{11}-\lambda & b_{12} & 0 & 0 \\
b_{31} & 0 & b_{33}-\lambda & b_{34} \\
0 & b_{82} & b_{83} & b_{84} \\
0 & b_{92} & b_{93} & b_{94}
\end{array}\right|=0 \text {. }
$$

For non-zero $D_{l}$, four real roots $\lambda$ are obtained:

$$
\begin{gathered}
\lambda_{1}=u+x_{2} \delta w=u+w_{1}, \\
\lambda_{2}=u-x_{1} \delta w=u+w_{2}, \\
\lambda_{3}=b_{55}=u+\frac{(\gamma-1) \delta \theta}{b_{1}} a_{1} \delta w \\
\lambda_{4}=b_{66}=u+\left(f_{1}-x_{1}\right) \delta w=u+w .
\end{gathered}
$$


Case 7 Two-phase flow without temperature and turbulent length scale diffusions $\left(D_{T}=0=\right.$ $\left.D_{l}, D_{k} \neq 0\right)$. In this case, the characteristic equation is

$$
b_{28} b_{49} b_{611} D_{k}\left(b_{55}-\lambda\right)\left(b_{77}-\lambda\right)\left|\begin{array}{cccc}
b_{11}-\lambda & b_{12} & 0 & 0 \\
b_{31} & 0 & b_{33}-\lambda & b_{34} \\
0 & b_{82} & b_{83} & b_{84} \\
0 & b_{92} & b_{93} & b_{94}
\end{array}\right|=0
$$

i.e.,

$$
\frac{16}{9} \frac{1}{\rho^{3}} \frac{f_{1} f_{2}}{a_{1}} D_{k}\left(b_{55}-\lambda\right)\left(b_{77}-\lambda\right)\left|\begin{array}{cccc}
b_{11}-\lambda & b_{12} & 0 & 0 \\
b_{31} & 0 & b_{33}-\lambda & b_{34} \\
0 & b_{82} & b_{83} & b_{84} \\
0 & b_{92} & b_{93} & b_{94}
\end{array}\right|=0 .
$$

If $D_{k} \neq 0$, then the roots $\lambda$ are the same as in Case 6 :

$$
\begin{gathered}
\lambda_{1}=u+x_{2} \delta w=u+w_{1}, \\
\lambda_{2}=u-x_{1} \delta w=u+w_{2}, \\
\lambda_{3}=b_{55}=u+\frac{(\gamma-1) \delta \theta}{b_{1}} a_{1} \delta w, \\
\lambda_{4}=b_{77}=u+\left(f_{1}-x_{1}\right) \delta w=u+w .
\end{gathered}
$$

Case 8 Two-phase flow without any diffusions $\left(D_{T}=D_{l}=D_{k}=0\right)$. In this case, the characteristic equation is

$$
b_{28} b_{49}\left(b_{55}-\lambda\right)\left(b_{66}-\lambda\right)\left(b_{77}-\lambda\right)\left|\begin{array}{cccc}
b_{11}-\lambda & b_{12} & 0 & 0 \\
b_{31} & 0 & b_{33}-\lambda & b_{34} \\
0 & b_{82} & b_{83} & b_{84} \\
0 & b_{92} & b_{93} & b_{94}
\end{array}\right|=0
$$

i.e.,

$$
\frac{1}{\rho^{2}}\left(b_{55}-\lambda\right)\left(b_{66}-\lambda\right)\left(b_{77}-\lambda\right)\left|\begin{array}{cccc}
b_{11}-\lambda & b_{12} & 0 & 0 \\
b_{31} & 0 & b_{33}-\lambda & b_{34} \\
0 & b_{82} & b_{83} & b_{84} \\
0 & b_{92} & b_{93} & b_{94}
\end{array}\right|=0
$$

For nonzero $\eta^{*}$, five real roots $\lambda$ are obtained:

$$
\begin{gathered}
\lambda_{1}=u+x_{2} \delta w=u+w_{1}, \\
\lambda_{2}=u-x_{1} \delta w=u+w_{2}, \\
\lambda_{3}=b_{55}=u+\frac{(\gamma-1) \delta \theta}{b_{1}} a_{1} \delta w, \\
\lambda_{4}=b_{66}=u+\left(f_{1}-x_{1}\right) \delta w=u+w, \\
\lambda_{5}=b_{77}=u+\left(f_{1}-x_{1}\right) \delta w=u+w .
\end{gathered}
$$

Up to now we found that the turbulent kinetic energy and length scale diffusions $\left(D_{k}\right.$ and $D_{l}$ ) have the same impacts on the characteristics of the model, the existence or vanishing of either one results in the same characteristics. This same behaviour is also found for the real and turbulent viscosities $\left(\eta\right.$ and $\eta_{d}$ ), i.e., the vanishing of either one results in the same 
characteristics. Thus $\eta^{*} \neq 0$ always gives two real roots: $\lambda_{1}=u+w_{1}$ and $\lambda_{2}=u+w_{2}$. $D_{T}=0$ produces a third real root: $\lambda_{3}=u+\frac{(\gamma-1) \delta \theta}{b_{1}} a_{1} \delta w . D_{k}=0$ and $D_{l}=0$ each produce a fourth real root: $\lambda_{4}=u+w$.

B. Zero viscosity $\left(\eta^{*}=0\right.$, so $\eta=0, \delta \eta=0$, and $\left.\eta_{d}=0\right)$.

Case 1 Two-phase flow with all diffusions $\left(D_{T} \neq 0, D_{l} \neq 0, D_{k} \neq 0\right)$. In this case, the characteristic equation is

$$
b_{510} b_{611} b_{712} D_{T} D_{k} D_{l}\left|\begin{array}{cccc}
b_{11}-\lambda & b_{12} & 0 & 0 \\
b_{21} & b_{22}-\lambda & b_{23} & b_{24} \\
b_{31} & 0 & b_{33}-\lambda & b_{34} \\
b_{41} & b_{42} & b_{43} & b_{44}-\lambda
\end{array}\right|=0
$$

i.e.,

$$
\frac{1}{\rho^{3}} \frac{(\gamma-1) T}{b_{1}} D_{T} D_{k} D_{l}\left|\begin{array}{cccc}
b_{11}-\lambda & b_{12} & 0 & 0 \\
b_{21} & b_{22}-\lambda & b_{23} & b_{24} \\
b_{31} & 0 & b_{33}-\lambda & b_{34} \\
b_{41} & b_{42} & b_{43} & b_{44}-\lambda
\end{array}\right|=0
$$

For non-zero $D_{T}, D_{k}$, and $D_{l}$, this equation requires

$$
\left|\begin{array}{cccc}
b_{11}-\lambda & b_{12} & 0 & 0 \\
b_{21} & b_{22}-\lambda & b_{23} & b_{24} \\
b_{31} & 0 & b_{33}-\lambda & b_{34} \\
b_{41} & b_{42} & b_{43} & b_{44}-\lambda
\end{array}\right|=0
$$

Substituting the $b_{i j}$ s into this equation gives

$$
\left|\begin{array}{cccc}
u-\lambda & \rho & 0 & 0 \\
\left(\frac{2}{3} k_{d}+a_{1} \delta w^{2}+b_{1}\right) / \rho & u-\lambda & a_{2} \delta w^{2}+b_{2} & 2 a_{1} \delta w \\
a_{1} \delta w / \rho & 0 & u+a_{2} \delta w-\lambda & a_{1} \\
a_{3}\left(\frac{2}{3} k_{d}+b_{1}\right) /\left(a_{1} \rho\right) & \delta w & -\delta w^{2}+\left(a_{3} / a_{1}\right) b_{2}+\left(c_{2} / a_{1}\right) & u+a_{2} \delta w-\lambda
\end{array}\right|=0
$$

By doing $(\operatorname{Row} 1) \times\left(-\frac{\delta w}{\rho}\right)+(\operatorname{Row} 4)$, this equation can be reduced to

$$
\left|\begin{array}{cccc}
u-\lambda & \rho & 0 & 0 \\
\frac{1}{\rho}\left(\frac{2}{3} k_{d}+a_{1} \delta w^{2}+b_{1}\right) & u-\lambda & a_{2} \delta w^{2}+b_{2} & 2 a_{1} \delta w \\
\frac{1}{\rho} a_{1} \delta w & 0 & u+a_{2} \delta w-\lambda & a_{1} \\
\frac{1}{\rho}\left[\left(\frac{2}{3} k_{d}+b_{1}\right) \frac{a_{3}}{a_{1}}-(u-\lambda) \delta w\right] & 0 & -\delta w^{2}+\frac{a_{3}}{a_{1}} b_{2}+\frac{c_{2}}{a_{1}} & u+a_{2} \delta w-\lambda
\end{array}\right|=0 .
$$

For convenience, let $y=u-\lambda$, and add $(\operatorname{Row} 3) \times(-2 \delta w)$ to (Row 2) giving

$$
\left|\begin{array}{cccc}
y & \rho & 0 & 0 \\
\frac{1}{\rho}\left(\frac{2}{3} k_{d}+a_{1} \delta w^{2}+b_{1}-2 a_{1} \delta w^{2}\right) & y & a_{2} \delta w^{2}+b_{2}-2\left(a_{2} \delta w+y\right) \delta w & 0 \\
\frac{1}{\rho} a_{1} \delta w & 0 & a_{2} \delta w+y & a_{1} \\
\frac{1}{\rho}\left[\frac{a_{3}}{a_{1}}\left(\frac{2}{3} k_{d}+b_{1}\right)-y \delta w\right] & 0 & -\delta w^{2}+\frac{a_{3}}{a_{1}} b_{2}+\frac{c_{2}}{a_{1}} & a_{2} \delta w+y
\end{array}\right|=0 \text {. }
$$

Add $(C o l .4) \times(-\delta w / \rho)$ to $(C o l .1)$ giving 


$$
\left|\begin{array}{cccc}
y & \rho & 0 & 0 \\
\frac{1}{\rho}\left(\frac{2}{3} k_{d}+a_{1} \delta w^{2}+b_{1}-2 a_{1} \delta w^{2}\right) & y & a_{2} \delta w^{2}+b_{2}-2\left(a_{2} \delta w+y\right) \delta w & 0 \\
0 & 0 & a_{2} \delta w+y & a_{1} \\
\frac{1}{\rho}\left[\frac{a_{3}}{a_{1}}\left(\frac{2}{3} k_{d}+b_{1}\right)-2 y \delta w-a_{2} \delta w^{2}\right] & 0 & -\delta w^{2}+\frac{a_{3}}{a_{1}} b_{2}+\frac{c_{2}}{a_{1}} & a_{2} \delta w+y
\end{array}\right|=0
$$

i.e.,

$$
\left|\begin{array}{cccc}
y & \rho & 0 & 0 \\
\frac{1}{\rho}\left(\frac{2}{3} k_{d}+b_{1}-a_{1} \delta w^{2}\right) & y & b_{2}-2 y \delta w-a_{2} \delta w^{2} & 0 \\
0 & 0 & a_{2} \delta w+y & a_{1} \\
\frac{1}{\rho}\left[\frac{a_{3}}{a_{1}}\left(\frac{2}{3} k_{d}+b_{1}\right)-2 y \delta w-a_{2} \delta w^{2}\right] & 0 & -\delta w^{2}+\frac{a_{3}}{a_{1}} b_{2}+\frac{c_{2}}{a_{1}} & a_{2} \delta w+y
\end{array}\right|=0 .
$$

Expanding according to the second row then gives

$$
\begin{gathered}
{\left[y^{2}-\left(\frac{2}{3} k_{d}+b_{1}-a_{1} \delta w^{2}\right)\right] \times\left|\begin{array}{cc}
a_{2} \delta w+y & a_{1} \\
-\delta w^{2}+\frac{a_{3}}{a_{1}} b_{2}+\frac{c_{2}}{a_{1}} & a_{2} \delta w+y
\end{array}\right|-} \\
a_{1}\left[b_{2}-2 y \delta w-a_{2} \delta w^{2}\right] \times\left|\begin{array}{cc}
y \\
\frac{1}{\rho}\left[\frac{a_{3}}{a_{1}}\left(\frac{2}{3} k_{d}+b_{1}\right)-2 y \delta w-a_{2} \delta w^{2}\right] & 0
\end{array}\right|=0,
\end{gathered}
$$

i.e.,

$$
\begin{gathered}
{\left[y^{2}-\left(\frac{2}{3} k_{d}+b_{1}-a_{1} \delta w^{2}\right)\right] \times\left[\left(a_{2} \delta w+y\right)^{2}+a_{1} \delta w^{2}-a_{3} b_{2}-c_{2}\right]-} \\
\left(b_{2}-2 y \delta w-a_{2} \delta w^{2}\right) \times\left[\frac{2}{3} a_{3} k_{d}+a_{3} b_{1}-2 a_{1} y \delta w-a_{1} a_{2} \delta w^{2}\right]=0
\end{gathered}
$$

This is a forth order polynomial in $y$ :

$$
y^{4}+A_{3} y^{3}+A_{2} y^{2}+A_{1} y+A_{0}=0
$$

where

$$
\begin{gathered}
A_{3}=2 a_{2} \delta w \\
A_{2}=\left(a_{2}^{2}-2 a_{1}\right) \delta w^{2}-a_{3} b_{2}-c_{2}-b_{1}-\frac{2}{3} k_{d} \\
A_{1}=\frac{4}{3} \delta w\left(a_{3}-a_{2}\right) k_{d}+2\left(a_{3} b_{1}-a_{2} b_{1}+a_{1} b_{2}\right) \delta w-2 a_{1} a_{2} \delta w^{3} \\
A_{0}=-\left(\frac{2}{3} k_{d}-a_{1} \delta w^{2}+b_{1}\right)\left[\left(a_{1}+a_{2}^{2}\right) \delta w^{2}-a_{3} b_{2}-c_{2}\right]-\left(\frac{2}{3} a_{3} k_{d}+a_{3} b_{1}-a_{1} a_{2} \delta w^{2}\right)\left(b_{2}-a_{2} \delta w^{2}\right) .
\end{gathered}
$$

In order to determine the properties (either real or complex) of the roots of this quartic, we use the following useful lemma (i.e., the W-lemma):

Lemma 1 (W). Consider the real quartic

$$
P(y)=y^{4}+A_{3} y^{3}+A_{2} y^{2}+A_{1} y+A_{0} \text {. }
$$

Necessary and sufficient conditions for $P$ to have four real roots are that $d P / d y$ have three real roots, $y_{1} \leq y_{2} \leq y_{3}$, such that the following three inequalities are satisfied

$$
P\left(y_{1}\right) \leq 0, \quad P\left(y_{2}\right) \geq 0, \text { and } P\left(y_{3}\right) \leq 0 .
$$


The plan of the proof becomes evident when the graph of a typical quartic is considered: roughly the graph has the shape of a " $W$ " with the three local extrema occuring at $y_{1}, y_{2}$, $y_{3}$. The proof can be found in $[11,12]$.

To apply the Lemma to equation (208), we must find the roots of the following cubic equation:

$$
P^{\prime} \equiv \frac{1}{4} \frac{d P}{d y}=y^{3}+B_{2} y^{2}+B_{1} y+B_{0}
$$

where

$$
\begin{aligned}
B_{2}=\frac{3}{4} A_{3} & =\frac{3}{2} a_{2} \delta w \\
B_{1}=\frac{1}{2} A_{2} & =\left(a_{2}^{2}-2 a_{1}\right) \delta w^{2}-\frac{1}{2}\left(a_{3} b_{2}+c_{2}+b_{1}\right)-\frac{1}{3} k_{d} \\
& =-\frac{1}{2}\left(a_{3} b_{2}+b_{1}\right)(1+\alpha) \\
B_{0}=\frac{1}{4} A_{1} & =\frac{1}{3} \delta w\left(a_{3}-a_{2}\right) k_{d}+\frac{1}{2}\left(a_{3} b_{1}-a_{2} b_{1}+a_{1} b_{2}-a_{1} a_{2} \delta w^{2}\right) \delta w \\
& =\frac{1}{2}\left(a_{3} b_{1}-a_{2} b_{1}+a_{1} b_{2}\right) \delta w(1+\beta) .
\end{aligned}
$$

Here

$$
\begin{aligned}
\alpha & \equiv\left[\frac{2}{3} k_{d}+c_{2}-\left(a_{2}^{2}-2 a_{1}\right) \delta w^{2}\right] /\left(a_{3} b_{2}+b_{1}\right) \\
& =\frac{a_{1}}{a_{1}+a_{3}^{2}}\left[\frac{2}{3}\left(1+\frac{f_{1} f_{2}}{x_{1} x_{2}}\right) \frac{k_{d}}{b_{1}}-\left(a_{2}^{2}-2 a_{1}\right) \frac{\delta w^{2}}{b_{1}}\right] \\
\beta & \equiv\left[\frac{2}{3}\left(a_{3}-a_{2}\right) k_{d}-a_{1} a_{2} \delta w^{2}\right] /\left(a_{3} b_{1}-a_{2} b_{1}+a_{1} b_{2}\right) \\
& =\frac{2\left(a_{3}-a_{2}\right)}{3\left(2 a_{3}-a_{2}\right)} \frac{k_{d}}{b_{1}}-\frac{a_{1} a_{2}}{2 a_{3}-a_{2}} \frac{\delta w^{2}}{b_{1}} .
\end{aligned}
$$

Let $y=x-\frac{1}{3} B_{2}$, then Eq.(209) becomes

$$
x^{3}+C_{1} x+C_{0}=0
$$

where

$$
\begin{aligned}
C_{1} & =B_{1}-\frac{1}{3} B_{2}^{2} \\
& =-\frac{1}{2} \frac{a_{3}^{2}+a_{1}}{a_{1}} b_{1}\left(1+\alpha+\frac{3}{2} \frac{a_{1} a_{2}}{a_{1}+a_{3}^{2}} \frac{\delta w^{2}}{b_{1}}\right) \\
& =-\frac{1}{2} \frac{a_{3}^{2}+a_{1}}{a_{1}} b_{1}\left[1+\frac{a_{1}}{a_{1}+a_{3}^{2}}\left(\frac{2}{3}\left(1+\frac{f_{1} f_{2}}{x_{1} x_{2}}\right) \frac{k_{d}}{b_{1}}+\frac{1}{2} \frac{\delta w^{2}}{b_{1}}\right)\right] \\
& =-\frac{1}{2} \frac{a_{3}^{2}+a_{1}}{a_{1}} b_{1}\left(1+\gamma_{1}\right)
\end{aligned}
$$


and

$$
\begin{aligned}
C_{0} & =\frac{1}{27}\left(2 B_{2}^{3}-9 B_{2} B_{1}+27 B_{0}\right) \\
& =\frac{1}{4} a_{2}^{3} \delta w^{3}+\left[\frac{1}{4} \frac{a_{3}^{2}-a_{1}}{a_{1}} a_{2}+a_{3}+\frac{1}{4} \frac{a_{3}^{2}}{a_{1}} a_{2} \alpha-\frac{1}{2} a_{2}\left(\beta-\frac{1}{2} \alpha\right)+a_{3} \beta\right] \delta w b_{1} \\
& =\left(\frac{1}{4} \frac{a_{3}^{2}-a_{1}}{a_{1}} a_{2}+a_{3}\right) \delta w b_{1}\left(1+\gamma_{2}\right),
\end{aligned}
$$

in which $\gamma_{1}$ and $\gamma_{2}$ are respectively defined as

$$
\begin{gathered}
\gamma_{1}=\frac{a_{1}}{a_{1}+a_{3}^{2}}\left[\frac{2}{3}\left(1+\frac{f_{1} f_{2}}{x_{1} x_{2}}\right) \frac{k_{d}}{b_{1}}+\frac{1}{2} \frac{\delta w^{2}}{b_{1}}\right], \\
\gamma_{2}=\frac{1}{\frac{1}{4} \frac{a_{3}^{2}-a_{1}}{a_{1}} a_{2}+a_{3}}\left[\frac{1}{4} a_{2}^{3} \frac{\delta w^{2}}{b_{1}}+\frac{1}{4} \frac{a_{3}^{2}}{a_{1}} a_{2} \alpha-\frac{1}{2} a_{2}\left(\beta-\frac{1}{2} \alpha\right)+a_{3} \beta\right] \\
=\frac{1}{\frac{1}{4} \frac{a_{3}^{2}-a_{1}}{a_{1}} a_{2}+a_{3}}\left[\frac{1}{4} a_{2}^{3} \frac{\delta w^{2}}{b_{1}}+\frac{1}{4} a_{2} \frac{a_{3}^{2}+a_{1}}{a_{1}} \alpha+\frac{1}{2}\left(2 a_{3}-a_{2}\right) \beta\right] \\
=\frac{1}{\frac{1}{4} \frac{a_{3}^{2}-a_{1}}{a_{1}} a_{2}+a_{3}} \cdot \frac{1}{6} \cdot\left[a_{2}\left(1+\frac{f_{1} f_{2}}{x_{1} x_{2}}\right)+2\left(a_{3}-a_{2}\right)\right] \frac{k_{d}}{b_{1}} .
\end{gathered}
$$

For solution let

$$
E=\left[-\frac{C_{0}}{2}+\left(\frac{C_{0}^{2}}{4}+\frac{C_{1}^{3}}{27}\right)^{1 / 2}\right]^{1 / 3} \quad \text { and } \quad \tilde{E}=\left[-\frac{C_{0}}{2}-\left(\frac{C_{0}^{2}}{4}+\frac{C_{1}^{3}}{27}\right)^{1 / 2}\right]^{1 / 3},
$$

then the values of $x$ are given by

$$
\mathfrak{x}=E+\tilde{E}, \quad-\frac{E+\tilde{E}}{2}+\frac{E-\tilde{E}}{2} \sqrt{-3}, \quad-\frac{E+\tilde{E}}{2}-\frac{E-\tilde{E}}{2} \sqrt{-3} .
$$

Thus, the three roots $y$ of Eq.(209) will be

$$
\begin{gathered}
y_{3}=-\frac{1}{2} a_{2} \delta w+E+\tilde{E} \\
y_{2}=-\frac{1}{2} a_{2} \delta w-\frac{E+\tilde{E}}{2}+\frac{E-\tilde{E}}{2} \sqrt{-3} \\
y_{1}=-\frac{1}{2} a_{2} \delta w-\frac{E+\tilde{E}}{2}-\frac{E-\tilde{E}}{2} \sqrt{-3}
\end{gathered}
$$

For real $B_{2}, B_{1}$, and $B_{0}$, these three roots are all real when and only when

$$
\frac{C_{0}^{2}}{4}+\frac{C_{1}^{3}}{27} \leq 0
$$


since $E$ and $\tilde{E}$ are then complex conjugates. Substituting the expressions for $C_{0}$ and $C_{1}$ into the above condition gives

$$
\begin{aligned}
& \frac{C_{0}^{2}}{4}+\frac{C_{1}^{3}}{27} \\
= & \frac{1}{4}\left(\frac{1}{4} \frac{a_{3}^{2}-a_{1}}{a_{1}} a_{2}+a_{3}\right)^{2} \delta w^{2} b_{1}^{2}\left(1+\gamma_{2}\right)^{2}+\frac{1}{27}\left(-\frac{1}{2} \frac{a_{3}^{2}+a_{1}}{a_{1}} b_{1}\right)^{3}\left(1+\gamma_{1}\right)^{3} \\
= & -\frac{1}{6^{3}}\left(\frac{a_{3}^{2}+a_{1}}{a_{1}} b_{1}\right)^{3}\left\{1+3 \gamma_{1}+3 \gamma_{1}^{2}+\gamma_{1}^{3}-54 a_{1} \frac{\left[\frac{1}{4}\left(a_{3}^{2}-a_{1}\right) a_{2}+a_{1} a_{3}\right]^{2}\left(1+2 \gamma_{2}+\gamma_{2}^{2}\right)}{\left(a_{3}^{2}+a_{1}\right)^{3}} \frac{\delta w^{2}}{b_{1}}\right\} \\
= & -\frac{1}{6^{3}}\left(\frac{a_{3}^{2}+a_{1}}{a_{1}} b_{1}\right)^{3} G,
\end{aligned}
$$

where

$$
G=1+3 \gamma_{1}+3 \gamma_{1}^{2}+\gamma_{1}^{3}-54 a_{1} \frac{\left[\frac{1}{4}\left(a_{3}^{2}-a_{1}\right) a_{2}+a_{1} a_{3}\right]^{2}\left(1+2 \gamma_{2}+\gamma_{2}^{2}\right)}{\left(a_{3}^{2}+a_{1}\right)^{3}} \frac{\delta w^{2}}{b_{1}}
$$

For simplification, we introduce the following parameters:

$$
\xi \equiv \frac{a_{1}}{a_{3}^{2}+a_{1}} \frac{k_{d}}{b_{1}}, \quad \zeta \equiv \frac{a_{1}}{a_{3}^{2}+a_{1}} \frac{\delta w^{2}}{b_{1}}, \quad \theta \equiv \frac{\xi}{\zeta}=\frac{k_{d}}{\delta w^{2}}, \quad \theta^{\prime} \equiv\left(1+\frac{f_{1} f_{2}}{x_{1} x_{2}}\right) \theta .
$$

Then Eqs.(215)-(216) become

$$
\begin{gathered}
\gamma_{1}=\frac{2}{3}\left(1+\frac{f_{1} f_{2}}{x_{1} x_{2}}\right) \xi+\frac{1}{2} \zeta=\zeta\left(\frac{2}{3} \theta^{\prime}+\frac{1}{2}\right), \\
\gamma_{2}=\frac{2}{3} \frac{a_{3}^{2}+a_{1}}{\left(a_{3}^{2}-a_{1}\right) a_{2}+4 a_{1} a_{3}}\left(a_{2} \frac{f_{1} f_{2}}{x_{1} x_{2}}+2 f_{1}-1\right) \frac{k_{d}}{b_{1}}=\frac{2}{3} \frac{1}{\tilde{F}} \frac{a_{2} \frac{f_{1} f_{2}}{x_{1} x_{2}}+2 f_{1}-1}{1+\frac{f_{1} f_{2}}{x_{1} x_{2}}} \zeta \theta^{\prime} .
\end{gathered}
$$

Here

$$
\tilde{F}=\frac{\left(a_{3}^{2}-a_{1}\right) a_{2}+4 a_{1} a_{3}}{a_{3}^{2}+a_{1}}=\frac{\Gamma_{1}^{2} x_{1}-\Gamma_{2}^{2} x_{2}}{\Gamma_{1}^{2} x_{1}+\Gamma_{2}^{2} x_{2}}=\frac{x_{1}-d^{2} x_{2}}{x_{1}+d^{2} x_{2}}
$$

is a function of $x_{1}$ and $\Gamma_{i}(i=1,2)$, and we have used the expressions:

$$
\begin{gathered}
a_{3}=f_{1}-x_{1}=\frac{\left(\Gamma_{1}-\Gamma_{2}\right) x_{1} x_{2}}{\Gamma_{1} x_{1}+\Gamma_{2} x_{2}}, \quad \frac{a_{1}}{a_{3}}=\frac{\Gamma_{1} x_{1}+\Gamma_{2} x_{2}}{\Gamma_{1}-\Gamma_{2}}, \\
\frac{a_{1}}{a_{3}^{2}}=\frac{\left(\Gamma_{1} x_{1}+\Gamma_{2} x_{2}\right)^{2}}{\left(\Gamma_{1}-\Gamma_{2}\right)^{2} x_{1} x_{2}}, \text { and } d \equiv \frac{\Gamma_{2}}{\Gamma_{1}} .
\end{gathered}
$$

Incorporating these expressions into the function $G$ gives

$$
G=1+H_{1}\left(\frac{\delta w^{2}}{b_{1}}\right)+H_{2}\left(\frac{\delta w^{4}}{b_{1}^{2}}\right)+H_{3}\left(\frac{\delta w^{6}}{b_{1}^{3}}\right)
$$

where

$$
H_{1}=3 \gamma_{1}-\frac{54}{16} \tilde{F}^{2} \frac{\delta w^{2}}{b_{1}}=\zeta\left(2 \theta^{\prime}+\frac{3}{2}-\frac{54}{16} \tilde{F}^{2}\right)
$$




$$
\begin{aligned}
& H_{2}=3 \gamma_{1}^{2}-\frac{54}{16} \cdot 2 \gamma_{2} \zeta=\zeta^{2}\left[\frac{4}{3} \theta^{\prime 2}+\left(2-\frac{9}{2} \tilde{F} \frac{a_{2} \frac{f_{1} f_{2}}{x_{1} x_{2}}+2 f_{1}-1}{1+\frac{f_{1} f_{2}}{x_{1} x_{2}}}\right) \theta^{\prime}+\frac{3}{4}\right] \\
&=\zeta^{2}\left(\frac{4}{3} \theta^{\prime 2}+g_{1} \theta^{\prime}+\frac{3}{4}\right), \\
& H_{3}=\gamma_{1}^{3}-\frac{54}{16} \gamma_{2}^{2} \zeta=\zeta^{3}\left\{\frac{8}{27} \theta^{\prime 3}+\left[\frac{2}{3}-\frac{3}{2} a_{2}^{2}-6\left(a_{3}-a_{2}\right) \frac{a_{2} \frac{f_{1} f_{2}}{x_{1} x_{2}}+a_{3}}{\left(1+\frac{f_{1} f_{2}}{x_{1} x_{2}}\right)^{2}}\right] \theta^{\prime 2}+\frac{1}{2} \theta^{\prime}+\frac{1}{8}\right\} \\
&=\zeta^{3}\left(\frac{8}{27} \theta^{\prime 3}+g_{2} \theta^{\prime 2}+\frac{1}{2} \theta^{\prime}+\frac{1}{8}\right),
\end{aligned}
$$

in which $g_{1}$ and $g_{2}$ are respectively defined as

$$
\begin{aligned}
g_{1} & =2-\frac{9}{2} \tilde{F} \frac{\left.a_{2} \frac{f_{1} f_{2}}{x_{1} x_{2}}+2 f_{1}-1\right)}{1+\frac{f_{1} f_{2}}{x_{1} x_{2}}}=2-\frac{9}{2} \tilde{F}\left(a_{2}+2 \cdot \frac{a_{3}-a_{2}}{1+\frac{f_{1} f_{2}}{x_{1} x_{2}}}\right) \\
& =2-\frac{9}{2} \tilde{F} \tilde{G}_{1}, \quad \tilde{G}_{1} \equiv a_{2}+2 \cdot \frac{a_{3}-a_{2}}{1+\frac{f_{1} f_{2}}{x_{1} x_{2}}}, \\
g_{2} & =\frac{2}{3}-\frac{3}{2} a_{2}^{2}-6\left(a_{3}-a_{2}\right) \frac{a_{2} \frac{f_{1} f_{2}}{x_{1} x_{2}}+a_{3}}{\left(1+\frac{f_{1} f_{2}}{x_{1} x_{2}}\right)^{2}} \\
& =\frac{2}{3}-\frac{3}{2} a_{2}^{2}-6\left(a_{3}-a_{2}\right) \tilde{G}_{2}, \quad \tilde{G}_{2} \equiv \frac{a_{2} \frac{f_{1} f_{2}}{x_{1} x_{2}}+a_{3}}{\left(1+\frac{f_{1} f_{2}}{x_{1} x_{2}}\right)^{2}} .
\end{aligned}
$$

Defining

$$
H_{1} \equiv \zeta \cdot H_{1}^{\prime}, \quad H_{2} \equiv \zeta^{2} \cdot H_{2}^{\prime}, \quad H_{3} \equiv \zeta^{3} \cdot H_{3}^{\prime}
$$

further gives

$$
G=1+\zeta H_{1}^{\prime}+\zeta^{2} H_{2}^{\prime}+\zeta^{3} H_{3}^{\prime},
$$

in which the $H_{i}^{\prime}(i=1,2,3)$ are functions only of $\theta^{\prime}$ :

$$
\begin{aligned}
& H_{1}^{\prime}=2 \theta^{\prime}+\frac{3}{2}-\frac{54}{16} \tilde{F}^{2}, \\
& H_{2}^{\prime}=\frac{4}{3} \theta^{\prime 2}+g_{1} \theta^{\prime}+\frac{3}{4} \\
& H_{3}^{\prime}=\frac{8}{27} \theta^{\prime 3}+g_{2} \theta^{\prime 2}+\frac{1}{2} \theta^{\prime}+\frac{1}{8} .
\end{aligned}
$$

It is clear that the factor $\frac{a_{3}^{2}+a_{1}}{a_{1}} b_{1}$ in equation (222) is always positive for any values of $x_{1}$ and $f_{1}$, so inequality (221) will be satisfied if and only if $G\left(\zeta, \theta^{\prime}\right) \geq 0$ for all values of $x_{1}, f_{1}$, $\zeta$, and $\theta^{\prime}$. According to the definitions (224), $\xi, \zeta, \theta$, and $\theta^{\prime}$ are all nonnegative parameters.

Statement 1. $G \geq 0$ for any $\zeta \geq 0$ and $\theta^{\prime} \geq 0$.

Proof:

(i) $G=G_{\min }$ at $d=0$. 
If and only if there is a point at which $\tilde{F}, \tilde{G}_{1}$, and $\tilde{G}_{2}$ all reach their maxima, then the $H_{i}^{\prime} \mathrm{s}(i=1,2,3)$ will attain their minima, and so does $G$.

Since

$$
\frac{\partial \tilde{F}}{\partial x_{1}}=\frac{2 d^{2}}{\left(x_{1}+d^{2} x_{2}\right)^{2}} \text { and } \frac{\partial \tilde{F}}{\partial d}=-\frac{4 d x_{1} x_{2}}{\left(x_{1}+d^{2} x_{2}\right)^{2}},
$$

both $\frac{\partial \tilde{F}}{\partial x_{1}}=0$ and $\frac{\partial \tilde{F}}{\partial d}=0$ produce $\tilde{F}=\tilde{F}_{\max }=1$ when and only when $d=0$, and $\tilde{F}=\tilde{F}_{\min }=-1$ when and only when $d \rightarrow \infty$. Therefore at $d=0, H_{1}^{\prime}$ reaches its minimum:

$$
H_{1 \text { min }}^{\prime}=H_{1}^{\prime}(d=0)=2 \theta^{\prime}-\frac{15}{8} \text {. }
$$

Similarly, it is straightforward to show that the functions

$$
a_{3}-a_{2}=\frac{x_{1}^{2}-d x_{2}^{2}}{x_{1}+d x_{2}}, \text { and } \frac{f_{1} f_{2}}{x_{1} x_{2}}=\frac{d}{\left(x_{1}+d x_{2}\right)^{2}}
$$

will both have their extreme values at $d=0$ as follows:

$$
\left(a_{3}-a_{2}\right)_{\max }=\left.\left(a_{3}-a_{2}\right)\right|_{d=0}=x_{1} \text {, and }\left(\frac{f_{1} f_{2}}{x_{1} x_{2}}\right)_{\min }=\left.\frac{f_{1} f_{2}}{x_{1} x_{2}}\right|_{d=0}=0
$$

which results in a maximum of $\tilde{G}_{1}$ or a minimum of $g_{1}$, and a minimum of $H_{2}^{\prime}$ :

$$
\begin{gathered}
\tilde{G}_{1 \max }=\left.\tilde{G}_{1}\right|_{d=0}=x_{2}+x_{1}=1, \\
g_{1 \min }=g_{1}(d=0)=-\frac{5}{2}, \\
H_{2 \min }^{\prime}=H_{2}^{\prime}(d=0)=\frac{4}{3} \theta^{\prime 2}-\frac{5}{2} \theta^{\prime}+\frac{3}{4} .
\end{gathered}
$$

The same arguments apply to

$$
\tilde{G}_{2}=\frac{a_{2} \frac{f_{1} f_{2}}{x_{1} x_{2}}+a_{3}}{\left(1+\frac{f_{1} f_{2}}{x_{1} x_{2}}\right)^{2}}=\frac{a_{2}}{1+\frac{f_{1} f_{2}}{x_{1} x_{2}}}+\frac{a_{3}-a_{2}}{\left(1+\frac{f_{1} f_{2}}{x_{1} x_{2}}\right)^{2}}
$$

and $g_{2}$, i.e., at $d=0, \tilde{G}_{2}$ attains its maximum and $g_{2}$ its minimum:

$$
\tilde{G}_{2 \max }=\left.\tilde{G}_{2}\right|_{d=0}=x_{2} \text { and } g_{2 \min }=g_{2}(d=0)=-\frac{5}{6} \text {. }
$$

This gives

$$
H_{3 \min }^{\prime}=H_{3}^{\prime}(d=0)=\frac{8}{27} \theta^{\prime 3}-\frac{5}{6} \theta^{\prime 2}+\frac{1}{2} \theta^{\prime}+\frac{1}{8} .
$$

Therefore, we conclude that for any $\zeta \geq 0$ and $\theta^{\prime} \geq 0$, the following is always true

$$
G \geq G_{\min } \equiv G(d=0)=1+\zeta H_{1 m i n}^{\prime}+\zeta^{2} H_{2 m i n}^{\prime}+\zeta^{3} H_{3 m i n}^{\prime},
$$

i.e.,

$$
\begin{gathered}
G \geq G_{\min }, \\
G_{\min }=1+\zeta\left(2 \theta^{\prime}-\frac{15}{8}\right)+\zeta^{2}\left(\frac{4}{3} \theta^{\prime 2}-\frac{5}{2} \theta^{\prime}+\frac{3}{4}\right)+\zeta^{3}\left(\frac{8}{27} \theta^{\prime 3}-\frac{5}{6} \theta^{\prime 2}+\frac{1}{2} \theta^{\prime}+\frac{1}{8}\right) .
\end{gathered}
$$

(ii) $G_{\min } \geq 0$ for any $\zeta \geq 0$ and $\theta^{\prime} \geq 0$.

We rewrite $G_{\min }$ into the convenient form 


$$
G_{\min }=1+2\left(\theta^{\prime}-\frac{15}{16}\right) \zeta+\frac{4}{3}\left(\theta^{\prime}-\frac{3}{2}\right)\left(\theta^{\prime}-\frac{3}{8}\right) \zeta^{2}+\frac{8}{27}\left(\theta^{\prime}-\frac{3}{2}\right)^{2}\left(\theta^{\prime}+\frac{3}{16}\right) \zeta^{3} .
$$

From this expression, we see that $\theta^{\prime} \geq \frac{3}{2}$ gives all coefficients $\geq 0$, so $G_{\min } \geq 0$ for all $\zeta \geq 0$. Thus the only case we need to consider is $\theta^{\prime} \leq \frac{3}{2}$. To see the lower limit of $G_{m i n}$, perform

$$
\frac{\partial G_{\min }}{\partial \zeta}=2\left(\theta^{\prime}-\frac{15}{16}\right)+\frac{8}{3}\left(\theta^{\prime}-\frac{3}{2}\right)\left(\theta^{\prime}-\frac{3}{8}\right) \zeta+\frac{8}{9}\left(\theta^{\prime}-\frac{3}{2}\right)^{2}\left(\theta^{\prime}+\frac{3}{16}\right) \zeta^{2}=0 .
$$

This is a quadratic equation in $\zeta$, i.e., $a \zeta^{2}+b \zeta+c=0$. Because

$$
b^{2}-4 a c=\frac{9}{4} \theta^{\prime 2}-\frac{27}{4} \theta^{\prime}+\frac{81}{16}=\left(\frac{3}{2} \theta^{\prime}-\frac{9}{4}\right)^{2} \geq 0
$$

for any $\theta^{\prime}$, Eq.(237) will have two real roots:

$$
\zeta_{+}=-\frac{3}{2} \frac{\theta^{\prime}-\frac{15}{16}}{\left(\theta^{\prime}-\frac{3}{2}\right)\left(\theta^{\prime}+\frac{3}{16}\right)} \quad \text { and } \quad \zeta_{-}=-\frac{3}{2} \frac{1}{\theta^{\prime}-\frac{3}{2}}
$$

at which the second derivative of $G_{\min }$ with respect to $\zeta$

$$
\frac{\partial^{2} G_{\min }}{\partial \zeta^{2}}=\frac{8}{3}\left(\theta^{\prime}-\frac{3}{2}\right)\left(\theta^{\prime}-\frac{3}{8}\right)+\frac{16}{9}\left(\theta^{\prime}-\frac{3}{2}\right)^{2}\left(\theta^{\prime}+\frac{3}{16}\right) \zeta
$$

has the respective values

$$
\left.\frac{\partial^{2} G_{\min }}{\partial \zeta^{2}}\right|_{\zeta=\zeta_{+}}=\frac{3}{2}\left(\theta^{\prime}-\frac{3}{2}\right) \text { and }\left.\frac{\partial^{2} G_{\min }}{\partial \zeta^{2}}\right|_{\zeta=\zeta_{-}}=-\frac{3}{2}\left(\theta^{\prime}-\frac{3}{2}\right) .
$$

For $\theta^{\prime}<\frac{3}{2}$

$$
\left.\frac{\partial^{2} G_{\min }}{\partial \zeta^{2}}\right|_{\zeta=\zeta_{+}}<0 \text { and }\left.\frac{\partial^{2} G_{\min }}{\partial \zeta^{2}}\right|_{\zeta=\zeta_{-}}>0
$$

so $\zeta_{+}$gives the maximum of $G_{\min }$, and $\zeta_{-}$gives the minimum of the $G_{\min }$. In this situation the minimum of $G_{\min }$ is

$$
\begin{aligned}
G_{\min }^{\min }\left(\zeta_{-}\right) & =1+2\left(\theta^{\prime}-\frac{15}{16}\right) \zeta_{-}+\frac{4}{3}\left(\theta^{\prime}-\frac{3}{2}\right)\left(\theta^{\prime}-\frac{3}{8}\right) \zeta_{-}^{2}+\frac{8}{27}\left(\theta^{\prime}-\frac{3}{2}\right)^{2}\left(\theta^{\prime}+\frac{3}{16}\right) \zeta_{-}^{3} \\
& =1+\frac{\frac{3}{2}-\theta^{\prime}}{\theta^{\prime}-\frac{3}{2}}=0 .
\end{aligned}
$$

Thus, for any $\zeta \geq 0$ and $\theta^{\prime} \geq 0$, the following is always true

$$
G \geq G_{\min } \geq 0 \text {. }
$$

Therefore the necessary and sufficient condition (221) for equation (209) to have three real roots is satisfied unconditionally. Next we will examine the signs of the polynomial $P$ at the three real roots, i.e., Eqs.(218)-(220). 
To simplify our calculation, we consider

$$
\begin{gathered}
P(y)-P^{\prime}(y) \cdot y-\frac{A_{3}}{4} P^{\prime}(y) \\
=\left(\frac{1}{2} A_{2}-\frac{3}{16} A_{3}^{2}\right) y^{2}+\left(\frac{3}{4} A_{1}-\frac{1}{8} A_{2} A_{3}\right) y+A_{0}-\frac{1}{16} A_{1} A_{3} .
\end{gathered}
$$

For the roots $\left\{y_{i}\right\}$ of $P^{\prime}(y), P^{\prime}\left(y_{i}\right) \equiv 0$, and we have

$$
P\left(y_{i}\right)-P^{\prime}\left(y_{i}\right) \cdot y_{i}-\frac{A_{3}}{4} P^{\prime}\left(y_{i}\right) \equiv P\left(y_{i}\right),
$$

i.e.,

$$
P\left(y_{i}\right) \equiv\left(\frac{1}{2} A_{2}-\frac{3}{16} A_{3}^{2}\right) y_{i}^{2}+\left(\frac{3}{4} A_{1}-\frac{1}{8} A_{2} A_{3}\right) y_{i}+A_{0}-\frac{1}{16} A_{1} A_{3}
$$

in which the coefficients are calculated as follows:

$$
\begin{gathered}
\frac{1}{2} A_{2}-\frac{3}{16} A_{3}^{2}=-\frac{1}{2}\left(1+\frac{a_{3}^{2}}{a_{1}}\right) b_{1}\left[1+\zeta\left(\frac{2}{3} \theta^{\prime}+\frac{1}{2}\right)\right] \\
\frac{3}{4} A_{1}-\frac{1}{8} A_{2} A_{3}=\delta w b_{1}\left\{3 a_{3}-\frac{3}{2} a_{2}+\left(1+\frac{a_{3}^{2}}{a_{1}}\right)\left[\zeta \theta^{\prime}\left(\frac{a_{3}-a_{2}}{1+\frac{f_{1} f_{2}}{x_{1} x_{2}}}+\frac{1}{6} a_{2}\right)+\frac{1}{4} a_{2}(1-\zeta)\right]\right\}, \\
A_{0}-\frac{1}{16} A_{1} A_{3}=\left(1+\frac{a_{3}^{2}}{a_{1}}\right) b_{1}^{2} \zeta\left\{\zeta \theta^{\prime} \frac{a_{3}^{2}+a_{1}}{a_{1}}\left[\frac{1}{2} \frac{a_{2}\left(a_{3}-a_{2}\right)}{1+\frac{f_{1} f_{2}}{x_{1} x_{2}}}-\frac{2}{3} a_{1}\right]+\right. \\
\left.\frac{2}{3} \theta^{\prime} \frac{\frac{f_{1} f_{2}}{x_{1} x_{2}}}{1+\frac{f_{1} f_{2}}{x_{1} x_{2}}}\left[\frac{2}{3} \frac{a_{1}+a_{3}^{2}}{a_{1}\left(1+\frac{f_{1} f_{2}}{x_{1} x_{2}}\right)} \zeta \theta^{\prime}+1\right]+\frac{1}{4}\left(a_{1}+a_{3}^{2}\right) \zeta-\left[\left(a_{3}-a_{2}\right)^{2}+\frac{1}{2} a_{2} a_{3}+2 a_{1}-\frac{1}{4}\right]\right\}
\end{gathered}
$$

where we have used

$$
\begin{gathered}
A_{3}=2 a_{2} \delta w \\
A_{2}=-\left(1+\frac{a_{3}^{2}}{a_{1}}\right) b_{1}\left[1+\zeta\left(\frac{2}{3} \theta^{\prime}-a_{2}^{2}+2 a_{1}\right)\right] \\
A_{1}=2 \delta w b_{1}\left(2 a_{3}-a_{2}\right)\left[1+\frac{1}{2 a_{3}-a_{2}}\left(1+\frac{a_{3}^{2}}{a_{1}}\right) \zeta\left(\frac{2}{3} \frac{a_{3}-a_{2}}{1+\frac{f_{1} f_{2}}{x_{1} x_{2}}} \theta^{\prime}-a_{1} a_{2}\right)\right], \\
A_{0}=\left(\frac{a_{1}+a_{3}^{2}}{a_{1}} b_{1}\right)^{2} \zeta\left[\frac{2}{3} \zeta \theta^{\prime}\left(\frac{a_{2}\left(a_{3}-a_{2}\right)}{1+\frac{f_{1} f_{2}}{x_{1} x_{2}}}-a_{1}\right)+\right. \\
\left.\frac{2}{3} \theta^{\prime} \frac{\frac{f_{1} f_{2}}{x_{1} x_{2}}}{1+\frac{f_{1} f_{2}}{x_{1} x_{2}}}\left(\frac{\zeta}{3} \frac{\zeta \theta^{\prime}}{1+\frac{f_{1} f_{2}}{x_{1} x_{2}}}+\frac{a_{1}}{a_{1}+a_{3}^{2}}\right)+a_{1}^{2} \zeta-\left(a_{3}-a_{2}\right)^{2}-a_{1}\right] .
\end{gathered}
$$

For our analysis, we introduce

$$
\begin{gathered}
D \equiv\left|\sqrt{\left|\frac{C_{0}^{2}}{4}+\frac{C_{1}^{3}}{27}\right|}\right|=\frac{|\sqrt{6}|}{36}\left(\frac{a_{1}+a_{3}^{2}}{a_{1}} b_{1}\right)^{3 / 2} G^{1 / 2} \\
D^{\prime} \equiv\left|\sqrt{\frac{C_{0}^{2}}{4}+D^{2}}\right|=\frac{|\sqrt{6}|}{36}\left(\frac{a_{1}+a_{3}^{2}}{a_{1}} b_{1}\right)^{3 / 2}\left(1+\frac{1}{2} \zeta+\frac{2}{3} \zeta \theta^{\prime}\right)^{3 / 2}
\end{gathered}
$$


and

or

$$
\phi \equiv \arctan \left(\frac{D}{-C_{0} / 2}\right)
$$

$$
\begin{gathered}
\tan \phi \equiv \frac{D}{-C_{0} / 2}=-\frac{2 \sqrt{6}}{9} \frac{\left[\frac{\left(1+\gamma_{1}\right)^{3}}{\zeta}-\frac{27}{8} \tilde{F}^{2}\left(1+\gamma_{2}\right)^{2}\right]^{1 / 2}}{\tilde{F}\left(1+\gamma_{2}\right) \aleph} \\
\aleph= \begin{cases}+1 & \text { if } \delta w>0 \\
-1 & \text { if } \delta w<0 .\end{cases}
\end{gathered}
$$

Then $E$ and $\tilde{E}$ become

$$
\begin{aligned}
& E=\left(-\frac{C_{0}}{2}+i D\right)^{1 / 3}=D^{1 / 3} e^{i \phi / 3}, \\
& \tilde{E}=\left(-\frac{C_{0}}{2}-i D\right)^{1 / 3}=D^{1 / 3} e^{-i \phi / 3},
\end{aligned}
$$

where $i=\sqrt{-1}$. With these expressions, the roots (218)-(220), which are the extrema of $P(y)$, are given by

$$
\begin{gathered}
y_{3}=-\frac{1}{2} a_{2} \delta w+2 D^{\prime 1 / 3} \cos \frac{\phi}{3} \\
y_{2}=-\frac{1}{2} a_{2} \delta w-D^{\prime 1 / 3}\left(\cos \frac{\phi}{3}+\sqrt{3} \sin \frac{\phi}{3}\right)=-\frac{1}{2} a_{2} \delta w-2 D^{\prime 1 / 3} \cos \left(\frac{\phi}{3}-\frac{\pi}{3}\right) \\
y_{1}=-\frac{1}{2} a_{2} \delta w-D^{\prime 1 / 3}\left(\cos \frac{\phi}{3}-\sqrt{3} \sin \frac{\phi}{3}\right)=-\frac{1}{2} a_{2} \delta w-2 D^{\prime 1 / 3} \cos \left(\frac{\phi}{3}+\frac{\pi}{3}\right)
\end{gathered}
$$

If we define

$$
\tan \phi^{\prime} \equiv \frac{D}{C_{0} / 2} \quad \text { or } \quad \cos \phi^{\prime} \equiv \frac{C_{0} / 2}{D^{\prime}}=\frac{3 \sqrt{6}}{4} \frac{\tilde{F}\left(1+\gamma_{2}\right) \zeta^{1 / 2} \aleph}{\left(1+\frac{1}{2} \zeta+\frac{2}{3} \xi^{\prime}\right)^{3 / 2}}
$$

then $\cos \phi^{\prime}=-\cos \phi$ and $\phi=\phi^{\prime} \pm \pi$. Therefore for the choice of $\phi=\phi^{\prime}-\pi$, the above three roots can be rewritten

$$
\begin{aligned}
& y_{1}=-\frac{1}{2} a_{2} \delta w-2 D^{\prime 1 / 3} \cos \frac{\phi^{\prime}}{3} \\
& y_{2}=-\frac{1}{2} a_{2} \delta w+2 D^{\prime 1 / 3} \cos \left(\frac{\phi^{\prime}}{3}+\frac{\pi}{3}\right) \\
& y_{3}=-\frac{1}{2} a_{2} \delta w+2 D^{\prime 1 / 3} \cos \left(\frac{\phi^{\prime}}{3}-\frac{\pi}{3}\right) .
\end{aligned}
$$

In order to see the range of $\phi^{\prime}$, we let

$$
\frac{\partial \cos \phi^{\prime}}{\partial \zeta}=0
$$

This gives the equation

$$
\tilde{F}\left[\frac{1}{2} \frac{\zeta^{-1 / 2}}{\left(1+\frac{1}{2} \zeta+\frac{2}{3} \xi^{\prime}\right)^{3 / 2}}-\frac{3}{4} \frac{\zeta^{1 / 2}}{\left(1+\frac{1}{2} \zeta+\frac{2}{3} \xi^{\prime}\right)^{5 / 2}}\right]=0 .
$$


For nonzero $\tilde{F}$, the solutions are

$$
\zeta_{c}=1+\frac{2}{3} \xi^{\prime} \quad \text { or } \quad \zeta_{c}=\infty
$$

at which $\cos \phi^{\prime}$ reaches its extrema:

$$
\left(\cos \phi^{\prime}\right)_{c}=\frac{3 \sqrt{6}}{4} \frac{\tilde{F}\left(1+\gamma_{2}\right) \zeta_{c}^{1 / 2} \aleph}{\left(1+\frac{1}{2} \zeta_{c}+\frac{2}{3} \xi^{\prime}\right)^{3 / 2}} \quad \text { or } \quad\left(\cos \phi^{\prime}\right)_{c}=0, \quad \phi_{c}^{\prime}=\frac{(n+1) \pi}{2}, \quad n=0,1,2 \ldots
$$

In the case of $k_{d}=0$,

$$
\zeta_{c}=1 \quad \text { and } \quad\left(\cos \phi^{\prime}\right)_{c}=\tilde{F} \aleph .
$$

For $\delta w>0, \aleph=1,\left(\cos \phi^{\prime}\right)_{c}=\tilde{F}$. If $\delta w<0, \aleph=-1$, then $\left(\cos \phi^{\prime}\right)_{c}=-\tilde{F}$. Assuming that material 2 is the heavy one, so $0 \leq d \leq 1$, in this case, $\tilde{F}$ varies from $0(\mathrm{~d}=1)$ to $1(\mathrm{~d}=0)$. Then whether the extreme of $\cos \phi^{\prime}$ is maximium or minimium only depends on the sign of $\delta w$. Noting that $\partial \phi^{\prime} / \partial \zeta<0$, so for $\aleph=1,\left(\cos \phi^{\prime}\right)_{c} \equiv\left(\cos \phi^{\prime}\right)_{\max }=\tilde{F}$ and $\phi_{c}^{\prime}=\phi_{\min }$. For $\aleph=-1,\left(\cos \phi^{\prime}\right)_{c} \equiv\left(\cos \phi^{\prime}\right)_{\min }=-\tilde{F}$ and $\phi_{c}^{\prime}=\phi_{\max }$. Thereupon for all signs of $\delta w, \phi^{\prime}$ varies from $-\tilde{F}$ to $\tilde{F}$. In the extreme case: $\tilde{F}_{\max (\min )}=1(0)$, we can estimate the possible range for $\phi^{\prime}$ :

and

$$
0 \leq \phi^{\prime} \leq \frac{\pi}{2} \text { for } \delta w>0
$$

$$
\frac{\pi}{2} \leq \phi^{\prime} \leq \pi \text { for } \delta w<0 .
$$

If $\phi=\phi^{\prime}-\pi$, then for both $\aleph=1$ and $\aleph=-1, y_{1} \leq y_{2} \leq y_{3}$, while if $\phi=\phi^{\prime}+\pi$, then roots (247) will be permuted from $\left(y_{1}, y_{2}, y_{3}\right)$ to $\left(y_{3}, y_{1}, y_{2}\right)$.

Now we examine the signs of the polynomial $P$ at these three extrema.

$A$. $\delta w=0$. In this situation, there is no relative motion between the two fluids. Setting $\zeta=0$ reduces Eqs.(243-245) to

$$
\begin{gathered}
\frac{1}{2} A_{2}-\frac{3}{16} A_{3}^{2}=-\frac{1}{2}\left(\frac{a_{1}+a_{3}^{2}}{a_{1}} b_{1}\right)\left(1+\frac{2}{3} \xi^{\prime}\right), \\
\frac{3}{4} A_{1}-\frac{1}{8} A_{2} A_{3}=0 \\
A_{0}-\frac{1}{16} A_{1} A_{2}=\frac{2}{3}\left(\frac{a_{1}+a_{3}^{2}}{a_{1}} b_{1}^{2}\right) \frac{f_{1} f_{2}}{x_{1} x_{2}} \xi\left(\frac{2\left(a_{1}+a_{3}^{2}\right)}{3 a_{1}} \xi+1\right), \\
D^{\prime 1 / 3}=\frac{\sqrt{6}}{6}\left(\frac{a_{1}+a_{3}^{2}}{a_{1}} b_{1}\right)^{1 / 2}\left(1+\frac{2}{3} \xi^{\prime}\right)^{1 / 2}, \quad \phi^{\prime} \rightarrow \frac{\pi}{2}
\end{gathered}
$$

where $\xi^{\prime}=\left(1+\frac{f_{1} f_{2}}{x_{1} x_{2}}\right) \xi$ and $\xi$ is defined by Eq.(224). The three roots are then

$$
\begin{aligned}
& y_{1}=-\frac{\sqrt{2}}{2}\left(\frac{a_{1}+a_{3}^{2}}{a_{1}} b_{1}\right)^{1 / 2}\left(1+\frac{2}{3} \xi^{\prime}\right)^{1 / 2} \\
& y_{2}=0 \\
& y_{3}=\frac{\sqrt{2}}{2}\left(\frac{a_{1}+a_{3}^{2}}{a_{1}} b_{1}\right)^{1 / 2}\left(1+\frac{2}{3} \xi^{\prime}\right)^{1 / 2}
\end{aligned}
$$


and Eq.(242) becomes

$$
P\left(y_{i}\right)=\frac{a_{1}+a_{3}^{2}}{a_{1}} b_{1}\left[-\frac{1}{2}\left(1+\frac{2}{3} \xi^{\prime}\right) y_{i}^{2}+\frac{2}{3} b_{1} \frac{f_{1} f_{2}}{x_{1} x_{2}} \xi\left(\frac{2\left(a_{1}+a_{3}^{2}\right)}{3 a_{1}} \xi+1\right)\right] .
$$

In particular,

$$
\begin{aligned}
P\left(y_{1}\right)= & P\left(y_{3}\right)=\frac{a_{1}+a_{3}^{2}}{a_{1}} b_{1}\left[-\frac{1}{2}\left(1+\frac{2}{3} \xi^{\prime}\right) y_{1}^{2}+\frac{2}{3} b_{1} \frac{f_{1} f_{2}}{x_{1} x_{2}} \xi\left(\frac{2\left(a_{1}+a_{3}^{2}\right)}{3 a_{1}} \xi+1\right)\right] \\
= & \frac{a_{1}+a_{3}^{2}}{a_{1}} b_{1}\left[-\frac{1}{4} \frac{a_{1}+a_{3}^{2}}{a_{1}} b_{1}\left(1+\frac{2}{3} \xi^{\prime}\right)^{2}+\frac{4}{9} b_{1} \frac{f_{1} f_{2}}{x_{1} x_{2}} \frac{a_{1}+a_{3}^{2}}{a_{1}} \xi^{2}+\frac{2}{3} b_{1} \frac{f_{1} f_{2}}{x_{1} x_{2}} \xi\right] \\
= & \left(\frac{a_{1}+a_{3}^{2}}{a_{1}} b_{1}\right)^{2}\left[-\frac{1}{4}-\frac{\xi}{3}\left(1+\frac{f_{1} f_{2}}{x_{1} x_{2}}-\frac{2 a_{1}}{a_{1}+a_{3}^{2}} \frac{f_{1} f_{2}}{x_{1} x_{2}}\right)-\frac{\xi^{2}}{9}\left(1-\frac{f_{1} f_{2}}{x_{1} x_{2}}\right)^{2}\right] \\
= & -\left(\frac{a_{1}+a_{3}^{2}}{a_{1}} b_{1}\right)^{2}\left[\frac{1}{4}+\frac{\xi}{3}\left(1+\frac{a_{3}^{2}-a_{1}}{a_{3}^{2}+a_{1}} \frac{f_{1} f_{2}}{x_{1} x_{2}}\right)+\frac{\xi^{2}}{9}\left(1-\frac{f_{1} f_{2}}{x_{1} x_{2}}\right)^{2}\right], \\
& P\left(y_{2}\right)=\frac{2}{3}\left(\frac{a_{1}+a_{3}^{2}}{a_{1}} b_{1}^{2}\right) \frac{f_{1} f_{2}}{x_{1} x_{2}} \xi\left(\frac{2\left(a_{1}+a_{3}^{2}\right)}{3 a_{1}} \xi+1\right) .
\end{aligned}
$$

Clearly,

$$
P\left(y_{2}\right) \geq 0 \quad \forall \quad \xi \geq 0
$$

and since

i.e.,

$$
\frac{a_{3}^{2}-a_{1}}{a_{3}^{2}+a_{1}} \frac{f_{1} f_{2}}{x_{1} x_{2}} \geq \frac{-a_{1}}{a_{3}^{2}+a_{1}} \frac{f_{1} f_{2}}{x_{1} x_{2}} \geq-\frac{f_{1} f_{2}}{x_{1} x_{2}}
$$

$$
\frac{\xi}{3}\left(1+\frac{a_{3}^{2}-a_{1}}{a_{3}^{2}+a_{1}} \frac{f_{1} f_{2}}{x_{1} x_{2}}\right) \geq \frac{\xi}{3}\left(1-\frac{f_{1} f_{2}}{x_{1} x_{2}}\right)
$$

we also have

$$
P\left(y_{1}\right)=P\left(y_{3}\right) \leq-\left(\frac{a_{1}+a_{3}^{2}}{a_{1}} b_{1}\right)^{2}\left[\frac{1}{2}-\frac{\xi}{3}\left(1-\frac{f_{1} f_{2}}{x_{1} x_{2}}\right)\right]^{2} \leq 0 \quad \forall \quad \xi \geq 0 .
$$

Therefore according to Lemma 1, Eq.(208) has all real roots for $\delta w=0$.

$B . \delta w \neq 0$. In this situation, there are relative motions between the fluids. Physically, any relative motion in inviscid flow will inevitablely produce turbulence $\left(k_{d}\right)$; for example, the Kelvin-Helmhotz $(\mathrm{KH})$ instability will be generated simultaneously at the fluid interface with an exponential growth rate $\sim \frac{\sqrt{\rho_{1} \rho_{2}}}{\rho_{1}+\rho_{2}} \delta w$ and will directly lead to turbulence and result in nonzero turbulent viscosity $\eta_{d} \sim l \delta w$, where $l$ is the length scale of the turbulence. Thus, in principle, we will be back to the same situation as in Case 1 for $\eta^{*} \neq 0$, which we have shown always gives real characteristics.

However, if the generation process for turbulent viscosity or turbulent kinetic energy is slow, then at an early time, for a tiny $\eta_{d}$, we would think that a good approximation should be $\eta_{d}=0$. Therefore, in order to see quantitatively the effects of the turbulent kinetic energy on the characteristics of the model, we would need to consider a more generalized situation, i.e., $\delta w \neq 0, k_{d} \neq 0$, but $\eta_{d}=0$, although this might not be physically realistic.

In this situation, for convenience, we let

$$
D_{2}=\frac{1}{2} A_{2}-\frac{3}{16} A_{3}^{2}, \quad D_{1}=\frac{3}{4} A_{1}-\frac{1}{8} A_{2} A_{3}, \quad D_{0}=A_{0}-\frac{1}{16} A_{1} A_{2}
$$


then polynomial $P\left(y_{i}\right)$ becomes

$$
P\left(y_{i}\right)=D_{2} y_{i}^{2}+D_{1} y_{i}+D_{0}
$$

This has maximum value

$$
P\left(y_{i}\right)_{m a x}=-\frac{D_{1}^{2}}{4 D_{2}}+D_{0}
$$

at $y_{i c}=-\frac{D_{1}}{2 D_{2}}$. It is clear that as $\delta w \rightarrow \infty, k_{d} \rightarrow \infty, \eta_{d} \neq 0$, Eq.(208) will always have four real roots. Thus for nonzero $\delta w$, physically, the infinitesimal $\eta_{d}$ only can happen if $\delta w$ and $k_{d}$ are tiny. Therefore the possible region for unreal characteristics of the model will be in the neighborhood of $\delta w=0$ where $\eta_{d}$ is negligible. Hence, the behaviour of the fluid under the sufficiently small nonzero $\delta w$ will impose significant constraints to the model for having all real characteristics in all values of $\delta w$.

To get the constraint on the model in inviscid flow, we now consider the neighborhood of small $\delta w$, i.e., $0<\zeta \ll 1$. In this region,

$$
\phi^{\prime} \rightarrow \frac{\pi}{2}, \quad \cos \frac{\phi^{\prime}}{3} \rightarrow \frac{\sqrt{3}}{2}, \quad \cos \left(\frac{\phi^{\prime}}{3}+\frac{\pi}{3}\right) \rightarrow 0, \quad \cos \left(\frac{\phi^{\prime}}{3}-\frac{\pi}{3}\right) \rightarrow \frac{\sqrt{3}}{2} .
$$

Thus the three roots Eq.(247) become

$$
y_{1}=-\frac{1}{2} a_{2} \delta w-D^{\prime 1 / 3} \sqrt{3}, \quad y_{2}=-\frac{1}{2} a_{2} \delta w, \quad y_{3}=-\frac{1}{2} a_{2} \delta w+D^{\prime 1 / 3} \sqrt{3}
$$

Substituting $D^{\prime}$ into above gives

$$
\begin{gathered}
y_{1}=\sqrt{\frac{a_{1}+a_{3}^{2}}{a_{1}} b_{1}}\left[-\frac{1}{2} a_{2} \zeta^{1 / 2}-\left(\frac{1}{2}+\zeta\left(\frac{1}{3} \theta^{\prime}+\frac{1}{4}\right)\right)^{1 / 2}\right] \\
y_{2}=-\sqrt{\frac{a_{1}+a_{3}^{2}}{a_{1}} b_{1}} \cdot \frac{1}{2} a_{2} \zeta^{1 / 2} \\
y_{3}=\sqrt{\frac{a_{1}+a_{3}^{2}}{a_{1}} b_{1}}\left[-\frac{1}{2} a_{2} \zeta^{1 / 2}+\left(\frac{1}{2}+\zeta\left(\frac{1}{3} \theta^{\prime}+\frac{1}{4}\right)\right)^{1 / 2}\right]
\end{gathered}
$$

Note that $y_{1}<y_{2}<y_{3}$. As $\delta w \rightarrow 0$ (or $\zeta \rightarrow 0$ ),

$$
\begin{gathered}
D_{2} \rightarrow-\frac{1}{2}\left(\frac{a_{1}+a_{3}^{2}}{a_{1}} b_{1}\right)+O(\zeta), \\
D_{1} \rightarrow\left(\frac{a_{1}+a_{3}^{2}}{a_{1}} b_{1}\right)^{3 / 2} \zeta^{1 / 2}\left(3 a_{3}-\frac{5}{4} a_{2}+\frac{a_{2} a_{3}^{2}}{4 a_{1}}\right)+O\left(\zeta^{3 / 2}\right), \\
D_{0} \rightarrow O\left(\zeta^{k}\right), \quad k \geq 1 .
\end{gathered}
$$

Thus, for surfficiently tiny $\delta w$, the first term in Eq.(250) dominates, which leads to

$$
P\left(y_{1}\right) \sim P\left(y_{3}\right) \rightarrow-\frac{1}{4}\left(\frac{a_{1}+a_{3}^{2}}{a_{1}} b_{1}\right)^{2}+O\left(\zeta^{k}\right)<0 .
$$

According to Lemma 1, if $P\left(y_{1}\right)$ and $P\left(y_{3}\right)$ are negative, then the sufficient condition for $P(y)$ to have four real roots is

$$
P\left(y_{2}\right) \geq 0
$$


Substituting $y_{2}$ into Eq.(250), we have the constraint

$$
\begin{aligned}
P\left(y_{2}\right)= & \left(\frac{a_{1}+a_{3}^{2}}{a_{1}} b_{1}\right)^{2} \zeta\left\{-\frac{a_{2}^{2}}{8}\left(1+\frac{1}{2} \zeta+\frac{2}{3} \zeta \theta^{\prime}\right)\right. \\
& -\frac{a_{2} a_{1}}{2\left(a_{1}+a_{3}^{2}\right)}\left[3 a_{3}-\frac{3}{2} a_{2}+\frac{a_{1}+a_{3}^{2}}{a_{1}} \zeta \theta^{\prime}\left(\frac{1}{6} a_{2}+\frac{a_{3}-a_{2}}{1+\frac{f_{1} f_{2}}{x_{1} x_{2}}}\right)+\frac{a_{1}+a_{3}^{2}}{4 a_{1}} a_{2}(1-\zeta)\right] \\
& +\zeta \theta^{\prime}\left[\frac{a_{2}\left(a_{3}-a_{2}\right)}{2\left(1+\frac{f_{1} f_{2}}{x_{1} x_{2}}\right)}-\frac{2}{3} a_{1}\right]+\frac{2}{3} \theta \frac{f_{1} f_{2}}{x_{1} x_{2}}\left(\frac{2}{3} \zeta \theta+\frac{a_{1}}{a_{1}+a_{3}^{2}}\right) \\
& \left.+\frac{1}{4} a_{1} \zeta-\frac{a_{1}}{a_{1}+a_{3}^{2}}\left[\left(a_{3}-a_{2}\right)^{2}+\frac{1}{2} a_{2} a_{3}+2 a_{1}-\frac{1}{4}\right]\right\} \geq 0 .
\end{aligned}
$$

Combining the same terms in the constraint gives

$$
\begin{gathered}
\zeta\left[-\frac{a_{2}^{2}}{8}\left(1+\frac{1}{2} \zeta+\frac{2}{3} \zeta \theta^{\prime}\right)-\frac{3}{4} \frac{a_{1} a_{2}\left(2 a_{3}-a_{2}\right)}{a_{1}+a_{3}^{2}}-\frac{1}{12} a_{2}^{2} \zeta \theta^{\prime}-\frac{1}{8} a_{2}^{2}(1-\zeta)-\frac{2}{3} a_{1} \zeta \theta^{\prime}\right. \\
\left.+\frac{4}{9} \frac{f_{1} f_{2}}{x_{1} x_{2}} \zeta \theta^{2}+\frac{2}{3} \frac{f_{1} f_{2}}{x_{1} x_{2}} \frac{a_{1}}{a_{1}+a_{3}^{2}} \theta+\frac{1}{4} a_{1} \zeta-\frac{a_{1}}{a_{1}+a_{3}^{2}}\left(a_{3}^{2}+a_{2}^{2}-\frac{3}{2} a_{2} a_{3}+2 a_{1}-\frac{1}{4}\right)\right] \geq 0 .
\end{gathered}
$$

Noting that

$$
4 a_{1}+a_{2}^{2}=1
$$

and doing further expansion and combination, we have

$$
\begin{gathered}
\zeta\left[-\frac{1}{4} a_{2}^{2}-\frac{3}{4} \frac{a_{1} a_{2}\left(2 a_{3}-a_{2}\right)}{a_{1}+a_{3}^{2}}+\frac{1}{16} \zeta-\frac{1}{6} \zeta \theta^{\prime}+\frac{4}{9} \frac{\frac{f_{1} f_{2}}{x_{1} x_{2}}}{\left(1+\frac{f_{1} f_{2}}{x_{1} x_{2}}\right)^{2}} \zeta \theta^{\prime 2}\right. \\
\left.+\frac{2}{3} \frac{\frac{f_{1} f_{2}}{x_{1} x_{2}}}{1+\frac{f_{1} f_{2}}{x_{1} x_{2}}} \frac{a_{1}}{a_{1}+a_{3}^{2}} \theta^{\prime}-\frac{a_{1}}{a_{1}+a_{3}^{2}}\left(a_{3}^{2}+a_{2}^{2}-\frac{3}{2} a_{2} a_{3}+2 a_{1}-\frac{1}{4}\right)\right] \geq 0
\end{gathered}
$$

where we have used $\theta^{\prime}=\left(1+\frac{f_{1} f_{2}}{x_{1} x_{2}}\right) \theta$. Combining the second and last terms in the above constraint gives

$$
-\frac{a_{1}}{a_{1}+a_{3}^{2}}\left(\frac{3}{4} a_{2}\left(2 a_{3}-a_{2}\right)+a_{3}^{2}+a_{2}^{2}-\frac{3}{2} a_{2} a_{3}+2 a_{1}-\frac{1}{4}\right)=-\frac{a_{1}}{a_{1}+a_{3}^{2}} \frac{1}{4}\left(a_{1}+a_{3}^{2}\right)=-\frac{1}{4} a_{1} .
$$

This further simplifies Eq.(259) into

or

$$
\zeta\left[-\frac{1}{4}+\frac{1}{16} \zeta-\frac{1}{6} \zeta \theta^{\prime}+\frac{2}{3} \frac{\frac{f_{1} f_{2}}{x_{1} x_{2}}}{1+\frac{f_{1} f_{2}}{x_{1} x_{2}}} \frac{a_{1}}{a_{1}+a_{3}^{2}} \theta^{\prime}+\frac{4}{9} \frac{\frac{f_{1} f_{2}}{z_{1} x_{2}}}{\left(1+\frac{f_{1} f_{2}}{x_{1} x_{2}}\right)^{2}} \zeta \theta^{\prime 2}\right] \geq 0,
$$

$$
\zeta\left\{\frac{4}{9} \frac{f_{1} f_{2}}{x_{1} x_{2}} \zeta \theta^{2}+\left[\frac{2}{3} \frac{a_{1}}{a_{1}+a_{3}^{2}} \frac{f_{1} f_{2}}{x_{1} x_{2}}-\frac{1}{6}\left(1+\frac{f_{1} f_{2}}{x_{1} x_{2}}\right) \zeta\right] \theta+\frac{1}{16} \zeta-\frac{1}{4}\right\} \geq 0 .
$$

For $\zeta>0$, this requires that

$$
\frac{4}{9} \frac{f_{1} f_{2}}{x_{1} x_{2}} \zeta \theta^{2}+\left[\frac{2}{3} \frac{a_{1}}{a_{1}+a_{3}^{2}} \frac{f_{1} f_{2}}{x_{1} x_{2}}-\frac{1}{6}\left(1+\frac{f_{1} f_{2}}{x_{1} x_{2}}\right) \zeta\right] \theta+\frac{1}{16} \zeta-\frac{1}{4} \geq 0 .
$$

In order to see the fundamental physics of this constraint, we now consider two special circumstances: 
(i) $k_{d}=0$. If we ignore the contribution from the turbulent motions of the fluid and set $\theta=0$, then the constraint becomes

$$
\frac{1}{16} \zeta-\frac{1}{4} \geq 0
$$

that is

$$
\zeta \geq 4 \text { or } \frac{\delta w^{2}}{b_{1}} \geq 4 \frac{a_{1}+a_{3}^{2}}{a_{1}} .
$$

This implies that in two-phase inviscid flow, if the turbulent kinetic energy is not taken into account, then there would exist a forbidden region for the velocity difference of two fluids, $0<\delta w^{2}<4 \frac{a_{1}+a_{3}^{2}}{a_{1}} b_{1}$, in which the model has complex characteristics and becomes illposed. This is consistent with the results obtained by Stewart and Wendroff ${ }^{[13]}$.

(ii) $k_{d} \neq 0$. For nonzero and nonnegative $k_{d}$, it is straightforward to solve inequality (262) for $\theta$ :

$$
\begin{gathered}
\theta_{-} \geq \theta \geq \theta_{+} \\
\theta_{ \pm}=\frac{3}{4 \zeta}\left\{-\frac{a_{1}}{a_{1}+a_{3}^{2}}+\frac{1+\frac{f_{1} f_{2}}{x_{1} x_{2}}}{4 \frac{f_{1} f_{2}}{x_{1} x_{2}}} \zeta \pm\left[\left(\frac{a_{1}}{a_{1}+a_{3}^{2}}-\frac{1+\frac{f_{1} f_{2}}{x_{1} x_{2}}}{4 \frac{f_{1} f_{2}}{x_{1} x_{2}}} \zeta\right)^{2}-\frac{\zeta\left(\frac{1}{4} \zeta-1\right)}{\frac{f_{1} f_{2}}{x_{1} x_{2}}}\right]^{1 / 2}\right\} \\
=\frac{3 b_{1}}{4 \delta w^{2}}\left\{-1+\frac{1+\frac{f_{1} f_{2}}{x_{1} x_{2}}}{4 \frac{f_{1} f_{2}}{x_{1} x_{2}}} \frac{\delta w^{2}}{b_{1}} \pm\left[\left(1-\frac{1+\frac{f_{1} f_{2}}{x_{1} x_{2}}}{4 \frac{f_{1} f_{2}}{x_{1} x_{2}}} \frac{\delta w^{2}}{b_{1}}\right)^{2}-\frac{\frac{\delta w^{2}}{b_{1}}\left(\frac{1}{4} \frac{\delta w^{2}}{b_{1}}-\frac{a_{1}+a_{3}^{2}}{a_{1}}\right)}{\frac{f_{1} f_{2}}{x_{1} x_{2}}}\right]^{1 / 2}\right\} \\
=\frac{3 b_{1}}{4 \delta w^{2}}\left[-1+\frac{1+\frac{f_{1} f_{2}}{x_{1} x_{2}}}{4 \frac{f_{1} f_{2}}{x_{1} x_{2}}} \frac{\delta w^{2}}{b_{1}} \pm\left(1-\frac{1+\frac{f_{1} f_{2}}{x_{1} x_{2}}}{4 \frac{f_{1} f_{2}}{x_{1} x_{2}}} \cdot \frac{\delta w^{2}}{b_{1}}\right) \Delta\right]
\end{gathered}
$$

where

$$
\Delta=\left[1+\frac{\frac{a_{1}+a_{3}^{2}}{a_{1}} \frac{x_{1} x_{2}}{f_{1} x_{2}} \frac{\delta w_{1}^{2}}{b_{1}}}{\left(1-\frac{1}{4}\left(1+\frac{z_{1} x_{2}}{f_{1} f_{2}}\right) \frac{\delta w^{2}}{b_{1}}\right)^{2}}\right]^{1 / 2},
$$

and the high order term $O\left(\frac{\delta w_{1}^{4}}{b_{1}^{2}}\right)$ has been neglected. For small $\delta w$,

$$
\Delta \sim 1+\frac{1}{2} \frac{\frac{a_{1}+a_{3}^{2}}{a_{1}} \frac{x_{1} x_{2}}{f_{1} x_{2}} \frac{\delta w^{2}}{b_{1}}}{\left(1-\frac{1}{4}\left(1+\frac{x_{1} x_{2}}{f_{1} f_{2}}\right) \frac{\delta w^{2}}{b_{1}}\right)^{2}} \sim 1+\frac{1}{2} \frac{\left(a_{1}+a_{3}^{2}\right)}{a_{1}} \frac{x_{1} x_{2}}{f_{1} f_{2}} \frac{\delta w^{2}}{b_{1}} .
$$

Substituting $\theta_{ \pm}$into solution (264), we obtain that for $\theta \leq \theta_{-}$,

$$
\frac{k_{d}}{b_{1}} \leq \frac{3}{4}\left[-1+\frac{1+\frac{f_{1} f_{2}}{x_{1} x_{2}}}{4 \frac{f_{1} f_{2}}{x_{1} x_{2}}} \frac{\delta w^{2}}{b_{1}}-\left(1-\frac{1+\frac{f_{1} f_{2}}{x_{1} x_{2}}}{4 \frac{f_{1} f_{2}}{x_{1} x_{2}}} \cdot \frac{\delta w^{2}}{b_{1}}\right) \Delta\right],
$$

and for $\theta \geq \theta_{+}$,

$$
\frac{k_{d}}{b_{1}} \geq \frac{3}{4}\left[-1+\frac{1+\frac{f_{1} f_{2}}{x_{1} x_{2}}}{4 \frac{f_{1} f_{2}}{x_{1} x_{2}}} \frac{\delta w^{2}}{b_{1}}+\left(1-\frac{1+\frac{f_{1} f_{2}}{x_{1} x_{2}}}{4 \frac{f_{1} f_{2}}{x_{1} x_{2}}} \cdot \frac{\delta w^{2}}{b_{1}}\right) \Delta\right] .
$$


If $\delta w$ is small, neglecting the $\frac{\delta w^{2}}{b_{1}^{1}}$ term, Eq.(267) becomes

$$
\frac{k_{d}}{b_{1}} \leq \frac{3}{4}\left[-2+\frac{1}{2}\left(1-\frac{a_{3}^{2}}{a_{1}} \frac{x_{1} x_{2}}{f_{1} f_{2}}\right) \frac{\delta w^{2}}{b_{1}}\right]
$$

It is evident that for any nonnegative $k_{d}$, this constraint is unphysical because the second term is much less than the first term if $\zeta \ll 1$. Therefore the real physical constraint to the model will be given by Eq.(268), i.e.,

$$
\frac{k_{d}}{b_{1}} \geq \frac{3}{8} \cdot \frac{a_{1}+a_{3}^{2}}{a_{1}} \cdot \frac{x_{1} x_{2}}{f_{1} f_{2}} \cdot \frac{\delta w^{2}}{b_{1}} \quad \text { or } \quad k_{d} \geq \frac{3}{8} \cdot \frac{a_{1}+a_{3}^{2}}{a_{1}} \cdot \frac{x_{1} x_{2}}{f_{1} f_{2}} \cdot \delta w^{2},
$$

if $\delta w$ is small. This tells us that at very early times when the turbulent viscosity is neglible, the model has all real characteristics for all physical ranges of $\delta w$ when and only when the turbulent kinetic energy satisfies

$$
k_{d} \geq \frac{3}{4} \cdot \frac{a_{1}+a_{3}^{2}}{a_{1}} \cdot \frac{k_{o}}{f_{1} f_{2}}
$$

This imposes an initial constraint on the model. This constraint physically means that the disordered motion is generated simultaneously with the ordered motion. If the two fluids are the same $\left(w_{1}=w_{2}\right)$, or if one of the fluids is vacuum $\left(w_{1}=w_{2}=0\right)$, then $k_{o}=0$ and condition (270) is apparently satisfied. Therefore the model has all real characteristics.

When $\delta w$ is not small, physically the inevitable Kelvin-Helmhotz instability generated due to $\delta w$ will result in nonnegligible $k_{d}$ and $\eta_{d}$, in which case the model always gives real characteristics as shown in Case 1 of $\eta * \neq 0$. A more accurate constraint on the model for general $\delta w$ is discussed in Appendix B.

Therefore we now conclude that in inviscid flow, for zero relative motion between the fluids, the hybrid multifluid turbulent mix model always has all real charachteristics. If there are non zero relative motions between the fluids and if turbulence $\left(k_{d}\right)$, turbulent viscosity $\left(\eta_{d}\right)$, and relative motion $(\delta w)$ between fluids are generated simultaneously, then the hybrid multifluid turbulent mix model will also have real characteristics in all ranges of $\delta w$. If at early times, $\delta w$ is very small $(\zeta \ll 1)$ and $\eta_{d}$ is negligible, then the model will possess complex characteristics unless the turbulent kinetic energy is equal or greater than $\frac{3}{4} \cdot \frac{a_{1}+a_{3}^{2}}{a_{1}} \cdot \frac{k_{o}}{f_{1} f_{2}}$. Moreover, if the turbulent contribution is completely ignored $\left(k_{d}=0\right)$, then there always exists an unremovable range for $\delta w, 0<\delta w^{2}<\frac{4\left(a_{1}+a_{3}^{2}\right)}{a_{1}} b_{1}$, in which the model has complex roots and becomes illposed as displayed in all traditional pure multiphase flow models. It is apparent that taking into account the contributions of turbulence may dismiss the well known illposedness of the pure multiphase flow model in the inviscid case if and only if condition (270) is satisfied forever. Otherwise, the introduction of $k_{d}$ only narrows the illposed region and improves the traditional multiphase flow model but cannot eradicate the illposedness. So taking into account the contributions of turbulence is not enough to complete the physics of turbulent mixing processes - some fundamental physics is still missing. For example, molecular diffusion is known to play an important role at late times of mixing as observed in recent linear electric motor (LEM) experiments ${ }^{[14]}$. Whence, for a correct and complete discription of the dynamics of turbulent mixing, additional physical processes such as molecular diffusion have to be taken into account so as to bridge the 
scales from macro to micro smoothly and to allow all parameters to take all values without generating illposedness.

The dynamic evolution of $k_{d}$ is examined in Appendix C, which shows that for inviscid flow with small $\delta w$, the ratio $k_{d} / k_{o}$ always increases with time, so the hybrid turbulent mix model will have all real characteristics if the constraint (270) is satisfied initially.

In the remainder of this section, we will list the characteristic equations for all other cases in the limit of inviscid flow, although most of these equations can only be evaluated numerically. Apparently, Case 1 is the simplest and only one which can be analysed analytically in this family.

Case 2 Two-phase flow without temperature diffusion $\left(D_{T}=0, D_{k} \neq 0, D_{l} \neq 0\right)$. In this case, the characteristic equation is

$$
b_{712} b_{611} D_{k} D_{l}\left|\begin{array}{ccccc}
b_{11}-\lambda & b_{12} & 0 & 0 & 0 \\
b_{21} & b_{22}-\lambda & b_{23} & b_{24} & b_{25} \\
b_{31} & 0 & b_{33}-\lambda & b_{34} & 0 \\
b_{41} & b_{42} & b_{43} & b_{44}-\lambda & b_{45} \\
b_{51} & b_{52} & b_{53} & b_{54} & b_{55}-\lambda
\end{array}\right|=0
$$

i.e.,

$$
\frac{1}{\rho^{2}} D_{k} D_{l}\left|\begin{array}{ccccc}
b_{11}-\lambda & b_{12} & 0 & 0 & 0 \\
b_{21} & b_{22}-\lambda & b_{23} & b_{24} & b_{25} \\
b_{31} & 0 & b_{33}-\lambda & b_{34} & 0 \\
b_{41} & b_{42} & b_{43} & b_{44}-\lambda & b_{45} \\
b_{51} & b_{52} & b_{53} & b_{54} & b_{55}-\lambda
\end{array}\right|=0
$$

Case 3 Two-phase flow without turbulent kinetic energy diffusion $\left(D_{k}=0, D_{T} \neq 0, D_{l} \neq 0\right)$. In this case, the characteristic equation is

i.e.,

$$
b_{510} b_{712} D_{T} D_{l}\left|\begin{array}{ccccc}
b_{11}-\lambda & b_{12} & 0 & 0 & 0 \\
b_{21} & b_{22}-\lambda & b_{23} & b_{24} & b_{26} \\
b_{31} & 0 & b_{33}-\lambda & b_{34} & 0 \\
b_{41} & b_{42} & b_{43} & b_{44}-\lambda & b_{46} \\
b_{61} & b_{62} & b_{63} & b_{64} & b_{66}-\lambda
\end{array}\right|=0 \text {, }
$$

$$
\frac{1}{\rho^{2}} \frac{(\gamma-1) T}{b_{1}} D_{T} D_{l}\left|\begin{array}{ccccc}
b_{11}-\lambda & b_{12} & 0 & 0 & 0 \\
b_{21} & b_{22}-\lambda & b_{23} & b_{24} & b_{26} \\
b_{31} & 0 & b_{33}-\lambda & b_{34} & 0 \\
b_{41} & b_{42} & b_{43} & b_{44}-\lambda & b_{46} \\
b_{61} & b_{62} & b_{63} & b_{64} & b_{66}-\lambda
\end{array}\right|=0
$$

Case \& Two-phase flow without turbulent length scale diffusion $\left(D_{l}=0, D_{T} \neq 0, D_{k} \neq 0\right)$. In this case, the characteristic is

$$
b_{510} b_{611} D_{T} D_{k}\left(b_{77}-\lambda\right)\left|\begin{array}{cccc}
b_{11}-\lambda & b_{12} & 0 & 0 \\
b_{21} & b_{22}-\lambda & b_{23} & b_{24} \\
b_{31} & 0 & b_{33}-\lambda & b_{34} \\
b_{41} & b_{42} & b_{43} & b_{44}-\lambda
\end{array}\right|=0
$$


i.e.,

$$
\frac{1}{\rho^{2}} \frac{(\gamma-1) T}{b_{1}} D_{T} D_{k}\left(b_{77}-\lambda\right)\left|\begin{array}{cccc}
b_{11}-\lambda & b_{12} & 0 & 0 \\
b_{21} & b_{22}-\lambda & b_{23} & b_{24} \\
b_{31} & 0 & b_{33}-\lambda & b_{34} \\
b_{41} & b_{42} & b_{43} & b_{44}-\lambda
\end{array}\right|=0 .
$$

For non-zero $D_{T}$ and $D_{k}$, one of the roots is given by

$$
\lambda_{1}=b_{77}=u+\left(f_{1}-x_{1}\right) \delta w=u+w,
$$

the other roots are as same as in Case 1 of this class.

Case 5 Two-phase flow without turbulent diffusions $\left(D_{k}=0=D_{l}, D_{T} \neq 0\right)$. In this case, the characteristic equation is

$$
b_{510} D_{T}\left(b_{77}-\lambda\right)\left|\begin{array}{ccccc}
b_{11}-\lambda & b_{12} & 0 & 0 & 0 \\
b_{21} & b_{22}-\lambda & b_{23} & b_{24} & b_{26} \\
b_{31} & 0 & b_{33}-\lambda & b_{34} & 0 \\
b_{41} & b_{42} & b_{43} & b_{44}-\lambda & b_{46} \\
b_{61} & b_{62} & b_{63} & b_{64} & b_{66}-\lambda
\end{array}\right|=0 \text {, }
$$

i.e.,

$$
\frac{(\gamma-1) T}{\rho b_{1}}\left(b_{77}-\lambda\right)\left|\begin{array}{ccccc}
b_{11}-\lambda & b_{12} & 0 & 0 & 0 \\
b_{21} & b_{22}-\lambda & b_{23} & b_{24} & b_{26} \\
b_{31} & 0 & b_{33}-\lambda & b_{34} & 0 \\
b_{41} & b_{42} & b_{43} & b_{44}-\lambda & b_{46} \\
b_{61} & b_{62} & b_{63} & b_{64} & b_{66}-\lambda
\end{array}\right|=0
$$

It has one real root

$$
\lambda_{1}=b_{77}=u+\left(f_{1}-x_{1}\right) \delta w=u+w
$$

and the others are as same as in Case 3 of the class.

Case 6 Two-phase flow without temperature and turbulent kinetic energy diffusions $\left(D_{T}=\right.$ $0=D_{k}, D_{l} \neq 0$ ). In this case, the characteristic equation is

$$
b_{712} D_{l}\left|\begin{array}{cccccc}
b_{11}-\lambda & b_{12} & 0 & 0 & 0 & 0 \\
b_{21} & b_{22}-\lambda & b_{23} & b_{24} & b_{25} & b_{26} \\
b_{31} & 0 & b_{33}-\lambda & b_{34} & 0 & 0 \\
b_{41} & b_{42} & b_{43} & b_{44}-\lambda & b_{45} & b_{46} \\
b_{51} & b_{52} & b_{53} & b_{54} & b_{55}-\lambda & 0 \\
b_{61} & b_{62} & b_{63} & b_{64} & 0 & b_{66}-\lambda
\end{array}\right|=0
$$

i.e.,

$$
\frac{D_{l}}{\rho}\left|\begin{array}{cccccc}
b_{11}-\lambda & b_{12} & 0 & 0 & 0 & 0 \\
b_{21} & b_{22}-\lambda & b_{23} & b_{24} & b_{25} & b_{26} \\
b_{31} & 0 & b_{33}-\lambda & b_{34} & 0 & 0 \\
b_{41} & b_{42} & b_{43} & b_{44}-\lambda & b_{45} & b_{46} \\
b_{51} & b_{52} & b_{53} & b_{54} & b_{55}-\lambda & 0 \\
b_{61} & b_{62} & b_{63} & b_{64} & 0 & b_{66}-\lambda
\end{array}\right|=0 .
$$


Case 7 Two-phase flow without temperature and turbulent length scale diffusions $\left(D_{T}=0=\right.$ $D_{l}, D_{k} \neq 0$ ). In this case, the characteristic equation is

$$
b_{611} D_{k}\left(b_{77}-\lambda\right)\left|\begin{array}{ccccc}
b_{11}-\lambda & b_{12} & 0 & 0 & 0 \\
b_{21} & b_{22}-\lambda & b_{23} & b_{24} & b_{25} \\
b_{31} & 0 & b_{33}-\lambda & b_{34} & 0 \\
b_{41} & b_{42} & b_{43} & b_{44}-\lambda & b_{45} \\
b_{51} & b_{52} & b_{53} & b_{54} & b_{55}-\lambda
\end{array}\right|=0 \text {, }
$$

i.e.,

$$
\frac{D_{k}}{\rho}\left|\begin{array}{ccccc}
b_{11}-\lambda & b_{12} & 0 & 0 & 0 \\
b_{21} & b_{22}-\lambda & b_{23} & b_{24} & b_{25} \\
b_{31} & 0 & b_{33}-\lambda & b_{34} & 0 \\
b_{41} & b_{42} & b_{43} & b_{44}-\lambda & b_{45} \\
b_{51} & b_{52} & b_{53} & b_{54} & b_{55}-\lambda
\end{array}\right|=0
$$

Clearly, besides the real root

$$
\lambda_{1}=b_{77}=u+\left(f_{1}-x_{1}\right) \delta w=u+w
$$

all other roots are the same as in Case 2 of the class.

Case 8 Two-phase flow without any diffusions $\left(D_{T}=D_{l}=D_{k}=0\right)$. In this case, the characteristic equation is

$$
\left(b_{77}-\lambda\right)\left|\begin{array}{cccccc}
b_{11}-\lambda & b_{12} & 0 & 0 & 0 & 0 \\
b_{21} & b_{22}-\lambda & b_{23} & b_{24} & b_{25} & b_{26} \\
b_{31} & 0 & b_{33}-\lambda & b_{34} & 0 & 0 \\
b_{41} & b_{42} & b_{43} & b_{44}-\lambda & b_{45} & b_{46} \\
b_{51} & b_{52} & b_{53} & b_{54} & b_{55}-\lambda & 0 \\
b_{61} & b_{62} & b_{63} & b_{64} & 0 & b_{66}-\lambda
\end{array}\right|=0 .
$$

It has seven roots, one is

$$
\lambda_{1}=b_{77}=u+\left(f_{1}-x_{1}\right) \delta w=u+w,
$$

and the other six are the same as in it Case 6.

From above, we find that the characteristics of the model are affected by the turbulent kinetic energy and temperature diffusions. The presence or absence of both or either one will change the characteristics of the model. However the turbulence length scale diffusion has no effect on the characteristics of the model in the limit of inviscid flow.

\section{Discussion}

In this report, we have studied the characteristics of the new hybrid multifluid turbulent mix model. In particular, we have performed a thorough analysis of the characteristics of this model in the case of one dimensional two-phase flows under various physical situations. Through our studies, we find that the new hybrid multifluid turbulent mix model under the assumption of pressure and temperature equilibrium has all real characteristics if either real or turbulent viscosity is present. The characteristic values of the model are affected by the turbulent kinetic energy and temperature diffusions, but are unaffected by the turbulent length scale diffusion. In inviscid flow where the real viscosity vanishes, the hybrid multifluid turbulent mix model still has real characteristic values if the relative motion between the 
fluids is zero. For nonzero relative motions between the fluids, if the disordered motions (turbulence) and turbulent viscosity together are generated simultaneously with the nonzero relative motions, then this model again has all real characteristics for all values of $\delta w$.

However, if at early times $\delta w$ is very small and $\eta_{d}$ is negligible, then the model will possess complex characteristics in a certain physical range of $\delta w$ unless the turbulent kinetic energy is equal to or greater than $\frac{3}{4} \cdot \frac{a_{1}+a_{3}^{2}}{a_{1}} \cdot \frac{k_{o}}{f_{1} f_{2}}$. This constitutes a constraint on the model. In the extreme case where the disordered turbulent contribution is completely ignored $\left(k_{d}=0\right)$, there is an unremovable range for $\delta w, 0<\delta w^{2}<\frac{4\left(a_{1}+a_{3}^{2}\right)}{a_{1}} b_{1}$, in which the model has complex characteristics and becomes illposed as do traditional single-pressure multiphase flow models in the absence of dissipation.

It is clear that the addition of disordered diffusive motion to the two-phase flow equations provides a natural dissipation which reduces the well-known illposedness of the singlepressure two-phase flow equations in the inviscid limit. However, the illposedness is only removed if constraint (270) is satisfied, a condition we have not been able to prove. Thus the hybrid multifluid turbulent mix model must still be missing some crucial physical processes. Molecular diffusion is one such process. Nevertheless, this model in its present form is well-posed even for inviscid fluids if both $k_{d}$ and $\eta_{d}$ are kept nonzero when $\delta w$ is not zero.

In the past decades, the physical origin of the complex characteristics in inviscid singlepressure two-phase flow models has been discussed extensively. It is known that this illposedness can be removed by the addition of viscosity or by letting the pressures of the two phases differ. Now we have shown that the addition of disordered turbulent kinetic energy $k_{d}$ with its associated turbulent viscosity $\eta_{d}$ will also remove the illposedness. In fact, even when $\eta_{d}=0$, the illposedness is removed if $k_{d}$ satisfies condition (270). Note that the disordered turbulent motions are physically necessary as the late-time decay products of the ordered drift motions even if they aren't needed to produce well-posed equations. For example, it is not adquate to obtain well-posed equations by simply letting the phase pressures differ since, in the absence of cohesive forces such as surface tension, a pressure imbalance can only be sustained by the ram pressures of the drift motions, and these drift motions decay away at late time.

Finally we would like to point out that this model only contains one drag frequency and one turbulent length scale for simplicity. A real mixing process probably involves a spectrum of both $\omega$ and $l$, and the materials eventually must mix at a microscopic scale. Therefore continuing improvements to this model are still ongoing. Nevertheless, the important advantage of this model is that its evolution equations are symmetrical in all materials and are formulated with vectors. This distinctive character allows the model to be applied to systems that contain any numbers of materials and spatial dimensions.

\section{ACKNOWLEDGMENTS}

This work was performed under the auspices of the U.S. Department of Energy by the Los Alamos National Laboratory under contract number W-7405-ENG-36. 


\section{REFERENCES}

[1] V.A. Andronov, S.M. Bakhrakh, E.E. Meshkov, V.V. Nikiforov, A.V. Pevnitskï, and A.I. Tolshmyyakov, "Turbulent mixing at contact surface accelerated by shock waves", Sov. Phys. JETP 44 (1976) 424-427.

[2] V. A. Andronov, S.M. Bakhrakh, E.E. Meshkov, V.V. Nikiforov, A.V. Pevnitskii, and A.I. Tolshmyyakov, "An experimental investigation and numerical modeling of turbulent mixing in one-dimensional flows", Doklady (Phys. Sect. in Engl. Trans.) 27 (May 1982) 393-396.

[3] D. Besnard, F.H. Harlow, and R. Rauenzahn, "Conservation and transport properties of turbulence with large density variations", Los Alamos National Laboratory report LA-10811-MS (February 1987).

[4] D. Besnard, F.H. Harlow, R. Rauenzahn, and C. Zemach, "Turbulence transport equations for variable density turbulence and their relationship to two-field models", Los Alamos National Laboratory report LA-12303-MS (June 1992).

[5] D.L. Youngs, "Numerical simulation of turbulent mixing by Rayleigh-Taylor instability", Physica D12 (1984) 32-44.

[6] D. Besnard and F.H. Harlow, "Turbulence in two-field incompressible flow", Los Alamos National Laboratory report LA-10187-MS (May 1985).

[7] D. Besnard, J.F. Haas, and R. Rauenzehn, "Statistical modeling of shock-interface interaction", Physica D37 (1989) 227-247.

[8] D.L. Youngs, "Modeling turbulent mixing by Reyleigh-Teylor instability", Physica D37 (1989) $270-287$.

[9] C.W. Cranfill, "A multifuid turbulent-mix model", Los Alamos National Laboratory report LA-UR-91-403 (January 1991).

[10] C.W. Cranfill, "A new multifluid turbulent-mix model", Los Alamos National Laboretory report LA-UR-92-2484 (August 1992).

[11] V.H. Ransom and D.L. Hicks, "Hyperbolic two-pressure models for two-phese fiow", J. of computational Phys. 53 (1984) 124-151.

[12] D.L. Hicks, "Hyperbolic models for two-phase (or two-material) flow", Sandia National Laboratories, SAND810253 (1981).

[13] H.B. Stewart and B. Wendroff, "Two-phase flow: models and methods", J. of computational Phys. 58 (1984) 363-409.

[14] G. Dimonte and M. Schneider, "Turbulent Rayleigh-Taylor instability experiments with variable acceleration", Phy. Rev. E54 (1996) 3740-3743.

[15] J.D. Ramshaw, "Physical derivation of virtual mass terms in two-phase flow", UCRL-JC-127505 (1997). 


\section{Appendix A. Pressure nonequilibrium situation}

For mixtures not in pressure and temperature equilibrium, the different materials have different pressure and temperature fluctuations, $P_{j}^{\prime}$ and $T_{j}^{\prime}$. Under this physical circumstance, the evolution equations of the fluid system become

- Bulk fluid equations:

$$
\begin{gathered}
0=\frac{d}{d t} \rho+\rho \vec{\nabla} \cdot \vec{u}, \\
0=\rho \frac{d}{d t} \vec{u}+\vec{\nabla} \cdot(\mathbf{R}+\mathbf{P}), \\
0=\rho \frac{d}{d t} i+\vec{\nabla} \cdot\left(\vec{s}+\vec{q}+\vec{s}^{\prime}+\vec{q}^{\prime}\right)+(\mathbf{P}+\mathbf{Q}): \vec{\nabla} \vec{u}+P \vec{\nabla} \cdot \vec{w}-\rho\left(\epsilon+\epsilon^{\prime}\right) .
\end{gathered}
$$

- Multifluid interpenetration equations:

$$
\begin{gathered}
0=\rho \frac{d}{d t} x_{j}+\vec{\nabla} \cdot\left(\rho x_{j} \vec{w}_{j}\right), \\
0=\rho \frac{d}{d t}\left(x_{j} \vec{w}_{j}\right)+\rho x_{j} \vec{w}_{j} \cdot \vec{\nabla} \vec{u}+\vec{\nabla} \cdot\left(\rho x_{j} \vec{w}_{j}^{2}+f_{j} \mathbf{R}_{\mathrm{dj}}+f_{j} \Pi_{j}+f_{j} P_{j}^{\prime} \delta\right) \\
+f_{j} \vec{\nabla} P+\left(\omega+\omega^{\prime}\right) \rho x_{j} \vec{w}_{j}+\rho x_{j} \frac{d}{d t} \vec{u}
\end{gathered}
$$

- Single-fluid turbulence equations:

$$
\begin{aligned}
& 0=\rho \frac{d}{d t} k+\vec{\nabla} \cdot\left(\vec{\sigma}+\vec{\pi}+\vec{p}^{\prime}\right)+(\mathbf{R}-\mathbf{Q}): \vec{\nabla} \vec{u}+(\vec{\nabla} P) \cdot \vec{w}+\rho\left(\epsilon+\epsilon^{\prime}\right), \\
& 0=\rho \frac{d}{d t} k_{o}+\vec{\nabla} \cdot\left(\vec{\sigma}_{o}+\vec{\pi}_{o}+\vec{p}_{o}^{\prime}\right)+\left(\mathbf{R}_{o}-\mathbf{Q}\right): \vec{\nabla} \vec{u}+(\vec{\nabla} P) \cdot \vec{w} \\
& +\rho \phi_{o}+2\left(\omega+\omega^{\prime}\right) \rho k_{o}+\rho\left(\epsilon_{o}+\epsilon_{o}^{\prime}\right) \\
& 0=\rho \frac{d}{d t} k_{d}+\vec{\nabla} \cdot\left[\rho k_{d} \vec{w}-C_{k d}\left(\mathbf{D}_{\mathbf{d}}+\mathbf{D}_{\mathbf{d}}^{\prime}\right) \cdot \vec{\nabla} k_{d}+\vec{p}_{d}^{\prime}\right] \\
& +\rho \phi_{d}-2\left(\omega+\omega^{\prime}\right) \rho k_{o}+\rho\left(\epsilon_{d}+\epsilon_{d}^{\prime}\right) \text {, } \\
& 0=\rho \frac{d}{d t} l+\vec{\nabla} \cdot\left[\rho l \vec{w}-C_{l d}\left(\mathbf{D}_{\mathbf{d}}+\mathbf{D}_{\mathrm{d}}^{\prime}\right) \cdot \vec{\nabla} l\right]-C_{l o} \rho\left(l \vec{\nabla} \cdot \vec{u}+\sqrt{k_{o}+k_{d}}\right)
\end{aligned}
$$

Where

$$
\begin{gathered}
f_{j} P_{j}^{\prime}=<c_{j} P^{\prime}>, \quad\left\langle P^{\prime}>=\sum_{j} f_{j} P_{j}^{\prime}=0,\right. \\
\omega^{\prime} \rho x_{j} \vec{w}_{j} \equiv-<P^{\prime} \boldsymbol{\delta} \cdot \vec{\nabla} c_{j}>, \\
\mathbf{D}_{\mathbf{d}}+\mathbf{D}_{\mathbf{d}}^{\prime} \equiv\left(\omega+\omega^{\prime}\right)^{-1}\left(\mathbf{R}_{\mathbf{d}}+\mathbf{I}_{\mathrm{o}}\right), \\
\mathbf{D}_{\mathbf{d}}^{\prime} \simeq-\frac{\omega^{\prime}}{\omega} \mathbf{D}_{\mathbf{d}}=\sum_{j} f_{j} \mathbf{D}_{\mathbf{d j},}^{\prime} \quad \mathbf{D}_{\mathbf{d j}}^{\prime}=-\frac{\omega^{\prime}}{\omega} \mathbf{D}_{\mathbf{d j}}, \quad \text { when }\left|\omega^{\prime}\right| \ll \omega,
\end{gathered}
$$


THE CHARACTERISTIC ANALYSIS OF A HYBRID MULTIFLUID TURBULENT-MIX MODEL

$$
\begin{gathered}
\vec{p}^{\prime} \equiv<P^{\prime} \delta \cdot \vec{v}^{\prime}>=\vec{p}_{o}^{\prime}+\vec{p}_{d,}^{\prime}, \quad \vec{p}_{o}^{\prime} \equiv \sum_{j} f_{j} P_{j}^{\prime} \delta \cdot \vec{w}_{j,} \\
\vec{p}_{d}^{\prime} \equiv \sum_{j} f_{j} \vec{p}_{d j}^{\prime}, \quad f_{j} \vec{p}_{d j}^{\prime}=<c_{j} P^{\prime} \delta \cdot\left(\vec{v}^{\prime}-\vec{w}_{j}\right)>, \\
\vec{\sigma}_{d}+\vec{\pi}_{d}+\vec{p}_{d}^{\prime}=\rho k_{d} \vec{w}+\sum_{j} f_{j} \mathbf{R}_{d j} \cdot \vec{w}_{j}+\sum_{j} f_{j}\left(\vec{\sigma}_{d j}+\vec{\pi}_{d j}+\vec{p}_{d j}^{\prime}\right), \\
\vec{s}^{\prime} \equiv<\rho x \theta^{\prime} \vec{v}^{\prime}>\equiv \sum_{j} \rho x_{j} \theta_{j}^{\prime} \vec{w}_{j}+\sum_{j}<c_{j} \rho x \theta^{\prime}\left(\vec{v}^{\prime}-\vec{w}_{j}\right)> \\
\rho \epsilon^{\prime}=\rho \epsilon_{o}^{\prime}+\rho \epsilon_{d,}^{\prime}, \quad \rho \epsilon_{o}^{\prime} \equiv-\sum_{j} f_{j} P_{j}^{\prime} \delta: \vec{\nabla} \vec{w}_{j}=\sum_{j} \rho x_{j} \epsilon_{o j}^{\prime}, \\
\rho \epsilon_{d}^{\prime} \equiv-\sum_{j}<c_{j} P^{\prime} \delta: \vec{\nabla}\left(\vec{v}^{\prime}-\vec{w}_{j}\right)>=\sum_{j} \rho x_{j} \epsilon_{d j}, \\
\vec{\nabla} \cdot \vec{p}_{o}^{\prime}=\sum_{j} \vec{w}_{j} \cdot \vec{\nabla}\left(f_{j} P_{j}^{\prime}\right)-\rho \epsilon_{o}^{\prime}, \\
\vec{\nabla} \cdot \vec{p}_{d}^{\prime}=\sum_{j}<\left(\vec{v}^{\prime}-\vec{w}_{j}\right) \cdot \vec{\nabla}\left(c_{j} P^{\prime}\right)>-\rho \epsilon_{d}^{\prime},
\end{gathered}
$$

and the new turbulence length scale is defined as

$$
l \equiv k_{d} \sqrt{k_{o}+k_{d}} /\left(\epsilon_{d}+\epsilon_{d}^{\prime}\right) .
$$

The pressure and temperature fluctuations, $P_{j}^{\prime}$ and $T_{j}^{\prime}$, are determined by the equations of state of each material, as are the internal energy fluctuations. If the fluid system is highly conductive, then $T_{j}^{\prime}$ is neglegible, and the system is nearly in temperature equlibrium, so the pressure is only a function of the mass density:

$$
P+P^{\prime}=\psi(m)
$$

The average pressure in each material accordingly satisfies

$$
f_{j} P_{j}=f_{j} P+f_{j} P_{j}^{\prime}=f_{j} \psi_{j}\left(m_{j}\right),
$$

where $f_{j} \psi_{j}\left(m_{j}\right) \equiv<c_{j} \psi(m)>$, and $\psi_{j}$ is given by the equation of state of the $j$-th material. For a small mass density fluctuation, the pressure fluctuations can be approximated by a Taylor expansion about the mean mass density $\rho$ :

$$
P+P^{\prime}=\psi(\rho)+\psi_{m}(\rho)(m-\rho)+\frac{1}{2} \psi_{m m}(\rho)(m-\rho)^{2}+\ldots,
$$

where $\psi_{m}(\rho)=(\partial \psi / \partial m)_{m=\rho}, \psi_{m m}(\rho)=\left(\partial^{2} \psi / \partial m^{2}\right)_{m=\rho}$, and

$$
P=\psi(\rho)+\frac{1}{2} \psi_{m m}(\rho)<(m-\rho)^{2}>+\ldots
$$

To a second order approximation $O\left[(m-\rho)^{2}\right], P^{\prime}$ is given by

$$
P^{\prime} \simeq \psi_{m}(\rho)(m-\rho)+\frac{1}{2} \psi_{m m}(\rho)\left[(m-\rho)^{2}-<(m-\rho)^{2}>\right], \quad m=\rho x
$$


and the individual pressure fluctuations in each material are

$$
\begin{aligned}
f_{j} P_{j}^{\prime}= & <c_{j} P^{\prime}> \\
= & \psi_{m}(\rho)<c_{j}(m-\rho)>+\frac{1}{2} \psi_{m m}(\rho)<\left(c_{j}-f_{j}\right)(m-\rho)^{2}> \\
= & \psi_{m}(\rho)\left(x_{j}-f_{j}\right) \rho+ \\
& \frac{1}{2} \psi_{m m}(\rho)\left[-2\left(1-x_{j}\right)<\left(c_{j}-f_{j}\right)\left(x-x_{j}\right)>+<\left(c_{j}-f_{j}\right)\left(x-x_{j}\right)^{2}>\right] \rho^{2} \\
= & \psi_{m}(\rho)\left(x_{j}-f_{j}\right) \rho+\psi_{m m}(\rho)\left[\left(f_{j}-x_{j}\right)\left(1-x_{j}\right)+\frac{1}{2}<\left(c_{j}-f_{j}\right)\left(x-x_{j}\right)^{2}>\right] \rho^{2} .
\end{aligned}
$$

This further gives the expression for the total pressure of each material up to the second order approximation:

$$
\begin{aligned}
f_{j} P_{j} & \equiv f_{j} \psi_{j}\left(m_{j}\right) \\
& \simeq f_{j} P+\psi_{m}(\rho)\left(x_{j}-f_{j}\right) \rho+ \\
& \psi_{m m}(\rho)\left[\left(f_{j}-x_{j}\right)\left(1-x_{j}\right)+\frac{1}{2}<\left(c_{j}-f_{j}\right)\left(x-x_{j}\right)^{2}>\right] \rho^{2}
\end{aligned}
$$

In general, since $\left\langle\left(c_{j}-f_{j}\right)\left(\mathfrak{x}-x_{j}\right)^{2}>/ 2\right.$ is negligible compared to $\left(f_{j}-x_{j}\right)\left(1-x_{j}\right)$ in a fluid system with small fluctuations, this will further simplify $P_{j}$ to

$$
P_{j} \simeq P+f_{j}^{-1} \psi_{m}(\rho)\left(x_{j}-f_{j}\right) \rho+f_{j}^{-1} \psi_{m m}(\rho)\left(f_{j}-x_{j}\right)\left(1-x_{j}\right) \rho^{2} .
$$

This expression is valid for most materials. In some simple gaseous systems, e.g. ideal gases, the high-order terms will be exactly zero $\left(O\left[\geq(m-\rho)^{2}\right]=0\right)$, so we are able to obtain an exact expression for $P_{j}$

$$
P_{j}=\psi_{j}\left(m_{j}\right)=P+f_{j}^{-1} \psi_{m}(\rho)\left(x_{j}-f_{j}\right) \rho .
$$

Either of these expressions for $P_{j}$ together with the equations of the state of each material will uniquely determine the volume fraction $f_{j}$.

Thus, in the physical situation of $P_{j}^{\prime} \neq 0$ but $T_{j}^{\prime}=0$, the model has equations for all the independent dynamical variables $\left(\rho, \vec{u}, i, x_{j}, \vec{w}_{j}, k_{d}, l\right.$, and $\left.P_{j}^{\prime}\right)$. Therefore this new hybrid multifluid turbulent mix model is also mathematically closed up to the second-order Taylor expansion even if the fluid systems have a small departure from pressure equilibrium.

Similar to the pressure equilibrium case, applying the model to a one-dimensional system containing only two fluids gives the following evolution equations:

$$
\begin{gathered}
\frac{\partial \rho}{\partial t}+u \frac{\partial \rho}{\partial x}+\rho \frac{\partial u}{\partial x}=0 \\
\rho \frac{\partial u}{\partial t}+\left(\frac{2}{3} k_{d}+a_{1} \delta w^{2}\right) \frac{\partial \rho}{\partial x}+\rho u \frac{\partial u}{\partial x}+\rho a_{2} \delta w^{2} \frac{\partial x_{1}}{\partial x}+2 \rho a_{1} \delta w \frac{\partial \delta w}{\partial x}+ \\
\frac{2}{3} \rho \frac{\partial k_{d}}{\partial x}-\frac{4}{3} \frac{\partial \Phi}{\partial x}+\frac{\partial P}{\partial x}=0 \\
\rho \frac{\partial x_{1}}{\partial t}+a_{1} \delta w \frac{\partial \rho}{\partial x}+\rho\left(u+a_{2} \delta w\right) \frac{\partial x_{1}}{\partial x}+\rho a_{1} \frac{\partial \delta w}{\partial x}=0
\end{gathered}
$$


THE CHARACTERISTIC ANALYSIS OF A HYBRID MULTIFLUID TURBULENT-MIX MODEL

$$
\begin{aligned}
& \rho x_{1} \frac{\partial u}{\partial t}+\rho a_{2} \delta w \frac{\partial x_{1}}{\partial t}+\rho a_{1} \frac{\partial \delta w}{\partial t}+\left[\frac{2}{3} f_{1} k_{d}+a_{1}\left(1-x_{1}\right) \delta w^{2}\right] \frac{\partial \rho}{\partial x}+\rho x_{1}\left[u+\left(1-x_{1}\right) \delta w\right] \frac{\partial u}{\partial x}+ \\
& \rho\left[a_{2} u+\left(1-x_{1}\right)\left(1-3 x_{1}\right) \delta w\right] \delta w \frac{\partial x_{1}}{\partial x}+\rho a_{1}\left[u+2\left(1-x_{1}\right) \delta w\right] \frac{\partial \delta w}{\partial x}+ \\
& \frac{2}{3} f_{1} \rho \frac{\partial k_{d}}{\partial x}-\frac{4}{3} f_{1}\left[\frac{\partial \Phi}{\partial x}+\left(1-f_{1}\right) \frac{\partial \delta \Phi}{\partial x}\right]+f_{1} \frac{\partial P}{\partial x}+\frac{\partial}{\partial x}\left(f_{1} P_{1}^{\prime}\right)+ \\
& {\left[\frac{2}{3} \rho k_{d}-\frac{4}{3}\left(\Phi+\left(1-2 f_{1}\right) \delta \Phi\right)\right] \frac{\partial f_{1}}{\partial x}+\left(\omega+\omega^{\prime}\right) a_{1} \rho \delta w=0,} \\
& \rho \frac{\partial i}{\partial t}+a_{1} \delta \theta \delta w \frac{\partial \rho}{\partial x}+\left[P-\frac{4}{3}\left(\Pi_{o}+Q\right)\right] \frac{\partial u}{\partial x}+\left(a_{2} \rho \delta \theta+\frac{4}{3} Q-P\right) \delta w \frac{\partial x_{1}}{\partial x}+ \\
& {\left[a_{1} \rho \delta \theta+a_{3} P+f_{1} P_{1}^{\prime}-\frac{4}{3} Q a_{3}-\frac{4}{3} \delta Q f_{1}\left(1-f_{1}\right)\right] \frac{\partial \delta w}{\partial x}+\rho u \frac{\partial i}{\partial x}+} \\
& \frac{\partial s_{d}}{\partial x}+\frac{\partial q}{\partial x}+P \delta w \frac{\partial f_{1}}{\partial x}+\rho a_{1} \delta w \frac{\partial \delta \theta}{\partial x}-\rho\left(\epsilon_{d}+\epsilon_{d}^{\prime}\right)=0 \\
& \rho \frac{\partial k_{d}}{\partial t}+k_{d} a_{3} \delta w \frac{\partial \rho}{\partial x}+\left(\frac{2}{3} \rho k_{d}-\frac{4}{3} \Gamma_{d}\right) \frac{\partial u}{\partial x}-\left(\frac{5}{3} \rho k_{d}-\frac{4}{3} \Gamma_{d}\right) \delta w \frac{\partial x_{1}}{\partial x}+ \\
& {\left[\frac{5}{3} \rho k_{d} a_{3}-\frac{4}{3} \Gamma_{d} a_{3}-\frac{4}{3} f_{1}\left(1-f_{1}\right) \delta \Gamma_{d}\right] \frac{\partial \delta w}{\partial x}+\rho\left(u+a_{3} \delta w\right) \frac{\partial k_{d}}{\partial x}+} \\
& \frac{\partial J_{k}}{\partial x}+\rho k_{d} \delta w \frac{\partial f_{1}}{\partial x}-\left(\omega+\omega^{\prime}\right) \rho a_{1} \delta w^{2}+\rho \epsilon_{d}=0, \\
& \rho \frac{\partial l}{\partial t}+a_{3} l \delta w \frac{\partial \rho}{\partial x}-C_{l o} \rho l \frac{\partial u}{\partial x}-\rho l \delta w \frac{\partial x_{1}}{\partial x}+a_{3} \rho l \frac{\partial \delta w}{\partial x}+\rho\left(u+a_{3} \delta w\right) \frac{\partial l}{\partial x}+ \\
& \frac{\partial J_{l}}{\partial x}+\rho l \delta w \frac{\partial f_{1}}{\partial x}-C_{l o} \rho \sqrt{k_{o}+k_{d}}=0 .
\end{aligned}
$$

Here we have used $\rho \epsilon_{o}^{\prime}=-f_{1} P_{1}^{\prime} \partial \delta w / \partial x$. The auxiliary equations for the new fluxes including $P_{j}^{\prime}$ are given by

$$
\begin{gathered}
\Phi-\left(\eta+\eta_{d}\right)\left[\frac{\partial u}{\partial x}-\delta w \frac{\partial x_{1}}{\partial x}+\left(a_{3}+f_{1} f_{2} \frac{\delta \eta}{\eta+\eta_{d}}\right) \frac{\partial \delta w}{\partial x}\right]=0 \\
\delta \Phi-\delta \eta\left[\frac{\partial u}{\partial x}-\delta w \frac{\partial x_{1}}{\partial x}+\left(1-f_{1}-x_{1}+\frac{\eta+\eta_{d}}{\delta \eta}\right) \frac{\partial \delta w}{\partial x}\right]=0 \\
s_{d}+\left(D_{s}+D_{s}^{\prime}\right) \frac{\partial T}{\partial x}=0, \quad D_{s}^{\prime}=C_{i d}\left(\frac{\partial i}{\partial T}\right)_{\rho} D_{d x}^{\prime} \\
q+D_{q} \frac{\partial T}{\partial x}=0, \quad D_{q}=\kappa \\
J_{k}+\left(D_{k}+D_{k}^{\prime}\right) \frac{\partial k_{d}}{\partial x}=0, \quad D_{k}^{\prime}=C_{k d} D_{d x}^{\prime} \\
J_{l}+\left(D_{l}+D_{l}^{\prime}\right) \frac{\partial l}{\partial x}=0, \quad D_{l}^{\prime}=C_{l d} D_{d x}^{\prime}
\end{gathered}
$$


The coefficients $D_{k}^{\prime}, D_{l}^{\prime}$, and $D_{s}^{\prime}$ are, respectively, the corrections to the diffusion coefficients $D_{k}, D_{l}$, and $D_{s}$ due to the effects of $P_{j}^{\prime}$.

Up to the second-order approximation for $P^{\prime}$, we have

$$
\begin{aligned}
f_{1} P_{1}^{\prime} & \simeq \psi_{m}(\rho)\left(x_{1}-f_{1}\right) \rho+\psi_{m m}(\rho)\left(f_{1}-x_{1}\right) x_{2} \rho^{2} \\
& =a_{3} \rho\left[-\psi_{m}(\rho)+\psi_{m m}(\rho) \rho x_{2}\right]
\end{aligned}
$$

and

$$
\begin{aligned}
\frac{\partial}{\partial x}\left(f_{1} P_{1}^{\prime}\right)= & \rho\left(-\psi_{m}+\rho x_{2} \psi_{m m}\right) \frac{\partial f_{1}}{\partial x}+\rho\left[\psi_{m}-\rho\left(x_{2}+a_{3}\right) \psi_{m m}\right] \frac{\partial x_{1}}{\partial x}- \\
& a_{3}\left[\rho \psi_{m \rho}+\psi_{m}-x_{2} \rho\left(\rho \psi_{m m \rho}+2 \psi_{m m}\right)\right] \frac{\partial \rho}{\partial x}
\end{aligned}
$$

where we have used the notations that

$$
\psi_{m} \equiv \psi_{m}(\rho), \quad \psi_{m \rho} \equiv \partial \psi_{m}(\rho) / \partial \rho, \quad \psi_{m m} \equiv \psi_{m m}(\rho), \quad \psi_{m m \rho} \equiv \partial \psi_{m m}(\rho) / \partial \rho
$$

Incorporating these approximations into the equations for $\delta w$ and $i$ gives

$$
\begin{gathered}
\rho x_{1} \frac{\partial u}{\partial t}+\rho a_{2} \delta w \frac{\partial x_{1}}{\partial t}+\rho a_{1} \frac{\partial \delta w}{\partial t}+ \\
{\left[\frac{2}{3} f_{1} k_{d}+a_{1}\left(1-x_{1}\right) \delta w^{2}-a_{3}\left(\rho \psi_{m \rho}+\psi_{m}\right)+a_{3} x_{2} \rho\left(\rho \psi_{m m \rho}+2 \psi_{m m}\right)\right] \frac{\partial \rho}{\partial x}+} \\
\rho x_{1}\left[u+\left(1-x_{1}\right) \delta w\right] \frac{\partial u}{\partial x}+\rho\left[a_{2} u \delta w+\left(1-x_{1}\right)\left(1-3 x_{1}\right) \delta w^{2}+\rho \psi_{m}-\left(x_{2}+a_{3}\right) \rho^{2} \psi_{m m}\right] \frac{\partial x_{1}}{\partial x}+ \\
\rho a_{1}\left[u+2\left(1-x_{1}\right) \delta w\right] \frac{\partial \delta w}{\partial x}+\frac{2}{3} f_{1} \rho \frac{\partial k_{d}}{\partial x}-\frac{4}{3} f_{1}\left(\frac{\partial \Phi}{\partial x}+f_{2} \frac{\partial \delta \Phi}{\partial x}\right)+f_{1} \frac{\partial P}{\partial x}+ \\
{\left[\frac{2}{3} \rho k_{d}-\frac{4}{3}\left(\Phi+\left(1-2 f_{1}\right) \delta \Phi\right)-\rho \psi_{m}+x_{2} \rho^{2} \psi_{m m}\right] \frac{\partial f_{1}}{\partial x}+} \\
\left(\omega+\omega^{\prime}\right) a_{1} \rho \delta w=0 \\
\rho \frac{\partial i}{\partial t}+a_{1} \delta \theta \delta w \frac{\partial \rho}{\partial x}+\left[P-\frac{4}{3}\left(\Pi_{0}+Q\right)\right] \frac{\partial u}{\partial x}+\left(a_{2} \rho \delta \theta+\frac{4}{3} Q-P\right) \delta w \frac{\partial x_{1}}{\partial x}+ \\
{\left[a_{1} \rho \delta \theta+a_{3} P-a_{3} \rho \psi_{m}+a_{3} x_{2} \rho^{2} \psi_{m m}-\frac{4}{3} Q a_{3}-\frac{4}{3} \delta Q f_{1} f_{2}\right] \frac{\partial \delta w}{\partial x}+\rho u \frac{\partial i}{\partial x}+} \\
\frac{\partial s_{d}}{\partial x}+\frac{\partial q}{\partial x}+P \delta w \frac{\partial f_{1}}{\partial x}+\rho a_{1} \delta w \frac{\partial \delta \theta}{\partial x}-\rho\left(\epsilon_{d}+\epsilon_{d}^{\prime}\right)=0
\end{gathered}
$$

For ideal gases, $\psi_{m m}=0=\psi_{m m \rho}$, so these two equations will further reduce to

$$
\begin{gathered}
\rho x_{1} \frac{\partial u}{\partial t}+\rho a_{2} \delta w \frac{\partial x_{1}}{\partial t}+\rho a_{1} \frac{\partial \delta w}{\partial t}+\left[\frac{2}{3} f_{1} k_{d}+a_{1}\left(1-x_{1}\right) \delta w^{2}-a_{3}\left(\rho \psi_{m} \rho+\psi_{m}\right)\right] \frac{\partial \rho}{\partial x}+ \\
\rho x_{1}\left[u+\left(1-x_{1}\right) \delta w\right] \frac{\partial u}{\partial x}+\rho\left[a_{2} u \delta w+\left(1-x_{1}\right)\left(1-3 x_{1}\right) \delta w^{2}+\rho \psi_{m}\right] \frac{\partial x_{1}}{\partial x}+ \\
\rho a_{1}\left[u+2\left(1-x_{1}\right) \delta w\right] \frac{\partial \delta w}{\partial x}+\frac{2}{3} f_{1} \rho \frac{\partial k_{d}}{\partial x}-\frac{4}{3} f_{1}\left(\frac{\partial \Phi}{\partial x}+f_{2} \frac{\partial \delta \Phi}{\partial x}\right)+f_{1} \frac{\partial P}{\partial x}+ \\
{\left[\frac{2}{3} \rho k_{d}-\frac{4}{3}\left(\Phi+\left(1-2 f_{1}\right) \delta \Phi\right)-\rho \psi_{m}\right] \frac{\partial f_{1}}{\partial x}+\left(\omega+\omega^{\prime}\right) a_{1} \rho \delta w=0}
\end{gathered}
$$




$$
\begin{gathered}
\rho \frac{\partial i}{\partial t}+a_{1} \delta \theta \delta w \frac{\partial \rho}{\partial x}+\left[P-\frac{4}{3}\left(\Pi_{\circ}+Q\right)\right] \frac{\partial u}{\partial x}+\left(a_{2} \rho \delta \theta+\frac{4}{3} Q-P\right) \delta w \frac{\partial x_{1}}{\partial x}+ \\
\left(a_{1} \rho \delta \theta+a_{3} P-a_{3} \rho \psi_{m}-\frac{4}{3} Q a_{3}-\frac{4}{3} \delta Q f_{1} f_{2}\right) \frac{\partial \delta w}{\partial x}+\rho u \frac{\partial i}{\partial x}+\frac{\partial s_{d}}{\partial x}+ \\
\frac{\partial q}{\partial x}+P \delta w \frac{\partial f_{1}}{\partial x}+\rho a_{1} \delta w \frac{\partial \delta \theta}{\partial x}-\rho\left(\epsilon_{d}+\epsilon_{d}^{\prime}\right)=0
\end{gathered}
$$


Appendix B. General constraint on the model in inviscid flow

The initial constraint (270) to the model can be relaxed with the increase of $\delta w$. For general $\delta w$, the sufficient condition for $\mathrm{W}$ shape $P(y)$ having all real roots is given by

$$
P\left(y_{2}\right) \geq 0 \text {. }
$$

For convenience, we rewrite $y_{2}$ into

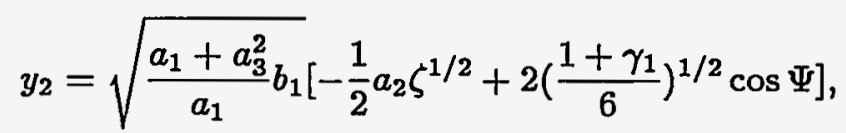

where $\gamma_{1}$ is given by Eq.(215) and $\Psi \equiv \frac{1}{3}\left(\phi^{\prime}+\pi\right)$. Thus

$$
y_{2}^{2}=\frac{a_{1}+a_{3}^{2}}{a_{1}} b_{1}\left[\frac{1}{4} a_{2}^{2} \zeta+\frac{2}{3}\left(1+\gamma_{1}\right) \cos ^{2} \Psi-a_{2} \sqrt{\frac{2}{3}\left(1+\gamma_{1}\right) \zeta} \cos \Psi\right]
$$

Substituting these expressions into Eq.(248) gives

$$
\begin{aligned}
& P\left(y_{2}\right)=-\frac{1}{2}\left(\frac{a_{1}+a_{3}^{2}}{a_{1}} b_{1}\right)^{2}\left(1+\gamma_{1}\right)\left[\frac{1}{4} a_{2}^{2} \zeta+\frac{2}{3}\left(1+\gamma_{1}\right) \cos ^{2} \Psi-a_{2} \sqrt{\frac{2}{3}\left(1+\gamma_{1}\right) \zeta} \cos \Psi\right]+ \\
& +\left(\frac{a_{1}+a_{3}^{2}}{a_{1}} b_{1}^{2}\right)\left[-\frac{1}{2} a_{2} \zeta^{1 / 2}+2\left(\frac{1+\gamma_{1}}{6}\right)^{1 / 2} \cos \Psi\right]\left\{3 a_{3}-\frac{3}{2} a_{2}+\left(1+\frac{a_{3}^{2}}{a_{1}}\right) \times\right. \\
& \left.\left[\zeta \theta^{\prime}\left(\frac{a_{3}-a_{2}}{1+\frac{f_{1} f_{2}}{x_{1} x_{2}}}+\frac{1}{6} a_{2}\right)+\frac{1}{4} a_{2}(1-\zeta)\right]\right\}+\left(1+\frac{a_{3}^{2}}{a_{1}}\right) b_{1}^{2} \zeta\left\{\zeta \theta^{\prime} \frac{a_{3}^{2}+a_{1}}{a_{1}} \times\right. \\
& {\left[\frac{a_{2}\left(a_{3}-a_{2}\right)}{2\left(1+\frac{f_{1} f_{2}}{x_{1} x_{2}}\right)}-\frac{2}{3} a_{1}\right]+\frac{2}{3} \theta^{\prime} \frac{\frac{f_{1} f_{2}}{x_{1} x_{2}}}{1+\frac{f_{1} f_{2}}{x_{1} x_{2}}}\left[\frac{2}{3} \frac{a_{1}+a_{3}^{2}}{a_{1}\left(1+\frac{f_{1} f_{2}}{x_{1} x_{2}}\right)} \zeta \theta^{\prime}+1\right]+} \\
& \left.\frac{1}{4}\left(a_{1}+a_{3}^{2}\right) \zeta-\left[\left(a_{3}-a_{2}\right)^{2}+\frac{1}{2} a_{2} a_{3}+2 a_{1}-\frac{1}{4}\right]\right\} \\
& =\left(\frac{a_{1}+a_{3}^{2}}{a_{1}} b_{1}\right)^{2}\left\{-\frac{1}{8} a_{2}^{2} \zeta\left(1+\gamma_{1}\right)-\frac{1}{3}\left(1+\gamma_{1}\right)^{2} \cos ^{2} \Psi+\left(1+\gamma_{1}\right)^{3 / 2} a_{2} \sqrt{\frac{\zeta}{6}} \cos \Psi+\right. \\
& \frac{a_{1} \zeta^{1 / 2}}{a_{1}+a_{3}^{2}}\left[-\frac{1}{2} a_{2} \zeta^{1 / 2}+2\left(\frac{1+\gamma_{1}}{6}\right)^{1 / 2} \cos \Psi\right]\left\{3 a_{3}-\frac{3}{2} a_{2}+\left(1+\frac{a_{3}^{2}}{a_{1}}\right) \times\right. \\
& \left.\left[\zeta \theta^{\prime}\left(\frac{a_{3}-a_{2}}{1+\frac{f_{1} f_{2}}{x_{1} x_{2}}}+\frac{1}{6} a_{2}\right)+\frac{1}{4} a_{2}(1-\zeta)\right]\right\}+\zeta^{2} \theta^{\prime}\left\{\left[\frac{a_{2}\left(a_{3}-a_{2}\right)}{2\left(1+\frac{f_{1} f_{2}}{x_{1} x_{2}}\right)}-\frac{2}{3} a_{1}\right]+\right. \\
& \frac{2 a_{1}}{3\left(a_{1}+a_{3}^{2}\right)} \frac{f_{1} f_{2}}{x_{1} x_{2}} \zeta \theta\left[\frac{2}{3} \frac{a_{1}+a_{3}^{2}}{a_{1}\left(1+\frac{f_{1} f_{2}}{x_{1} x_{2}}\right)} \zeta \theta^{\prime}+1\right]+ \\
& \left.\frac{a_{1}}{a_{1}+a_{3}^{2}} \zeta\left\{\frac{1}{4}\left(a_{1}+a_{3}^{2}\right) \zeta-\left[\left(a_{3}-a_{2}\right)^{2}+\frac{1}{2} a_{2} a_{3}+2 a_{1}-\frac{1}{4}\right]\right\}\right\} \geq 0 \text {. }
\end{aligned}
$$

Since

$$
\left(\frac{a_{1}+a_{3}^{2}}{a_{1}} b_{1}\right)^{2} \geq 0
$$


Eq.(321) thus implies that

$$
\begin{gathered}
-\frac{1}{8} a_{2}^{2} \zeta\left(1+\gamma_{1}\right)-\frac{1}{3}\left(1+\gamma_{1}\right)^{2} \cos ^{2} \Psi+\left(1+\gamma_{1}\right)^{3 / 2} a_{2} \sqrt{\frac{\zeta}{6}} \cos \Psi-\frac{3}{4} \frac{a_{1} a_{2}}{a_{1}+a_{3}^{2}} \zeta\left(2 a_{3}-a_{2}\right)- \\
\frac{1}{2} a_{2} \zeta^{2} \theta^{\prime}\left(\frac{a_{3}-a_{2}}{1+\frac{f_{1} f_{2}}{x_{1} x_{2}}}+\frac{1}{6} a_{2}\right)-\frac{1}{8} a_{2}^{2} \zeta(\zeta-1)+\frac{3 a_{1}\left(2 a_{3}-a_{2}\right)}{a_{1}+a_{3}^{2}}\left(\frac{1+\gamma_{1}}{6} \zeta\right)^{1 / 2} \cos \Psi+ \\
2\left(\frac{1+\gamma_{1}}{6} \zeta\right)^{1 / 2}\left(\frac{a_{3}-a_{2}}{1+\frac{f_{1} f_{2}}{x_{1} x_{2}}}+\frac{1}{6} a_{2}\right) \zeta \theta^{\prime} \cos \Psi+\frac{a_{2}(1-\zeta)}{2}\left(\frac{1+\gamma_{1}}{6} \zeta\right)^{1 / 2} \cos \Psi+ \\
\left(\frac{a_{2}\left(a_{3}-a_{2}\right)}{2\left(1+\frac{f_{1} f_{2}}{x_{1} x_{2}}\right)}-\frac{2}{3} a_{1}\right) \zeta^{2} \theta^{\prime}+\frac{4}{9} \frac{f_{1} f_{2}}{x_{1} x_{2}} \zeta^{2} \theta^{2}+\frac{2 a_{1}}{3\left(a_{1}+a_{3}^{2}\right)} \frac{f_{1} f_{2}}{x_{1} x_{2}} \zeta \theta+ \\
\frac{a_{1}}{a_{1}+a_{3}^{2}} \zeta\left[\frac{1}{4}\left(a_{1}+a_{3}^{2}\right) \zeta-a_{3}^{2}-a_{2}^{2}+\frac{3}{2} a_{2} a_{3}-2 a_{1}+\frac{1}{4}\right] \geq 0 .
\end{gathered}
$$

Combining the same terms togather in Eq.(322) reduces it to

$$
\begin{gathered}
-\frac{1}{4} a_{2}^{2} \zeta-\frac{1}{8} a_{2}^{2} \zeta\left(\gamma_{1}-\zeta\right)-\frac{1}{3}\left(1+\gamma_{1}\right)^{2} \cos ^{2} \Psi+\left(1+\gamma_{1}\right)^{3 / 2} a_{2} \sqrt{\frac{\zeta}{6}} \cos \Psi-\left(\frac{1}{12} a_{2}^{2}+\frac{2}{3} a_{1}\right) \eta^{2} \theta^{\prime} \\
\frac{3 a_{1}\left(2 a_{3}-a_{2}\right)}{a_{1}+a_{3}^{2}}\left(\frac{1+\gamma_{1}}{6} \zeta\right)^{1 / 2} \cos \Psi+2\left(\frac{1+\gamma_{1}}{6} \zeta\right)^{1 / 2}\left(\frac{a_{3}-a_{2}}{1+\frac{f_{1} f_{2}}{x_{1} x_{2}}}+\frac{1}{6} a_{2}\right) \zeta \theta^{\prime} \cos \Psi+ \\
\frac{a_{2}(1-\zeta)}{2}\left(\frac{1+\gamma_{1}}{6} \zeta\right)^{1 / 2} \cos \Psi+\frac{4}{9} \frac{f_{1} f_{2}}{x_{1} x_{2}} \zeta^{2} \theta^{2}+\frac{2 a_{1}}{3\left(a_{1}+a_{3}^{2}\right)} \frac{f_{1} f_{2}}{x_{1} x_{2}} \zeta \theta+ \\
\frac{a_{1}}{a_{1}+a_{3}^{2}} \zeta\left[\frac{3}{4} a_{2}^{2}+\frac{1}{4}\left(a_{1}+a_{3}^{2}\right) \zeta-\left(a_{3}^{2}+a_{2}^{2}+2 a_{1}-\frac{1}{4}\right)\right] \geq 0
\end{gathered}
$$

Using identity Eq.(258), the last term in above equation becomes

$$
\frac{a_{1}}{a_{1}+a_{3}^{2}} \zeta\left[\frac{3}{4} a_{2}^{2} \zeta+\frac{1}{4}\left(a_{1}+a_{3}^{2}\right) \zeta-\left(a_{3}^{2}+a_{2}^{2}+2 a_{1}-\frac{1}{4}\right) \zeta\right]=\frac{1}{4} a_{1} \zeta^{2}-a_{1} \zeta
$$

this togather with $\gamma_{1}$ 's expression further simplify Eq.(323) into

$$
\begin{gathered}
-\frac{1}{4} a_{2}^{2} \zeta-\frac{1}{6} a_{2}^{2} \zeta^{2} \theta^{\prime}+\frac{1}{16} \zeta^{2}-\frac{1}{3}\left(1+\gamma_{1}\right)^{2} \cos ^{2} \Psi+\left(\frac{1+\gamma_{1}}{6} \zeta\right)^{1 / 2} \cos \Psi\left[a_{2}\left(1+\gamma_{1}\right)+\right. \\
\frac{3 a_{1}\left(2 a_{3}-a_{2}\right)}{a_{1}+a_{3}^{2}}+ \\
\left.+\left(\frac{2\left(a_{3}-a_{2}\right)}{1+\frac{f_{1} f_{2}}{x_{1} x_{2}}}+\frac{1}{3} a_{2}\right) \zeta \theta^{\prime}+\frac{1}{2} a_{2}(1-\zeta)\right]+\frac{4}{9} \frac{f_{1} f_{2}}{x_{1} x_{2}} \zeta^{2} \theta^{2}+ \\
\frac{2 a_{1}}{3\left(a_{1}+a_{3}^{2}\right)} \frac{f_{1} f_{2}}{x_{1} x_{2}} \zeta \theta+\frac{1}{4} a_{1} \zeta^{2}-a_{1} \zeta \geq 0 .
\end{gathered}
$$

Again applying Eq.(258) and Eq.(225), we are able to further reduce the constraint equation to

$$
\begin{gathered}
\frac{1}{16} \zeta^{2}-\frac{1}{4} \zeta-\frac{1}{6} \zeta^{2} \theta^{\prime}-\frac{1}{3}\left(1+\gamma_{1}\right)^{2} \cos ^{2} \Psi+\left(\frac{1+\gamma_{1}}{6} \zeta\right)^{1 / 2} \cos \Psi\left[\frac{3}{2} \tilde{F}+\right. \\
\left.\left(\frac{2\left(a_{3}-a_{2}\right)}{1+\frac{f_{1} f_{2}}{x_{1} x_{2}}}+a_{2}\right) \zeta \theta^{\prime}\right]+\frac{4}{9} \frac{f_{1} f_{2}}{x_{1} x_{2}} \zeta^{2} \theta^{2}+\frac{2 a_{1}}{3\left(a_{1}+a_{3}^{2}\right)} \frac{f_{1} f_{2}}{x_{1} x_{2}} \zeta \theta \geq 0 .
\end{gathered}
$$


Substituting $\gamma_{1}$ into Eq.(338) gives

Noticing that

$$
\begin{gathered}
\frac{1}{16} \zeta^{2}-\frac{1}{4} \zeta-\frac{1}{6} \zeta^{2} \theta^{\prime}-\frac{1}{3}\left(1+\zeta+\frac{1}{4} \zeta^{2}+\frac{4}{3} \zeta \theta^{\prime}+\frac{1}{3} \zeta^{2} \theta^{\prime}+\frac{4}{9} \zeta^{2} \theta^{\prime 2}\right) \cos ^{2} \Psi+ \\
+\left(\frac{1}{6} \zeta+\frac{1}{12} \zeta^{2}+\frac{1}{9} \zeta^{2} \theta^{\prime}\right)^{1 / 2} \cos \Psi\left[\frac{3}{2} \tilde{F}+\left(\frac{2\left(a_{3}-a_{2}\right)}{1+\frac{f_{1} f_{2}}{x_{1} x_{2}}}+a_{2}\right) \zeta \theta^{\prime}\right]+ \\
\frac{4}{9} \frac{f_{1} f_{2}}{x_{1} x_{2}} \zeta^{2} \theta^{2}+\frac{2 a_{1}}{3\left(a_{1}+a_{3}^{2}\right)} \frac{f_{1} f_{2}}{x_{1} x_{2}} \zeta \theta \geq 0 .
\end{gathered}
$$

$$
\zeta \theta^{\prime}=\xi^{\prime}=\left(1+\frac{f_{1} f_{2}}{x_{1} x_{2}}\right) \xi, \quad \zeta \theta=\xi, \quad \zeta^{2} \theta^{\prime}=\zeta \xi^{\prime}, \quad \zeta^{2} \theta=\zeta \xi
$$

then Eq.(339) can be finally writen as

$$
\begin{gathered}
\frac{1}{16} \zeta^{2}-\frac{1}{4} \zeta-\frac{1}{6} \zeta \xi^{\prime}-\frac{1}{3}\left(1+\zeta+\frac{1}{4} \zeta^{2}+\frac{4}{3} \xi^{\prime}+\frac{1}{3} \zeta \xi^{\prime}+\frac{4}{9} \xi^{\prime 2}\right) \cos ^{2} \Psi+ \\
\left(\frac{1}{6} \zeta+\frac{1}{12} \zeta^{2}+\frac{1}{9} \zeta \xi^{\prime}\right)^{1 / 2}\left[\frac{3}{2} \tilde{F}+2\left(a_{3}-a_{2}\right) \xi+a_{2} \xi^{\prime}\right] \cos \Psi+ \\
\frac{4}{9} \frac{f_{1} f_{2}}{x_{1} x_{2}} \xi^{2}+\frac{2 a_{1}}{3\left(a_{1}+a_{3}^{2}\right)} \frac{f_{1} f_{2}}{x_{1} x_{2}} \xi \geq 0
\end{gathered}
$$

This equation provides a general constraint for the model to have all real characteristics even in inviscid flow. Clearly, at both $\delta w=0$ and $\delta w \rightarrow \infty$, Eq.(340) is satisfied because $\cos \Psi=0$ in both cases, this tells us that the model does have real characheristics in both situations. These are well consistent with the results that we obtained earlier. Meanwhile, we see that a large $\xi$ (i.e., large turbulent kinetic energy $k_{d}$ ) always assures the satisfaction of Eq.(340). However, it is obvious that condition (340) will break down if both $\delta w$ and $k_{d}$ are small especially in the early stage of the dynamic evolution of the system when the second term in the equation is dominant. For zero $\xi\left(k_{d}=0\right)$, Eq.(340) becomes

$$
\frac{1}{16} \zeta^{2}-\frac{1}{4} \zeta-\frac{1}{3}\left(1+\frac{\zeta}{2}\right)^{2} \cos ^{2} \Psi+\left(\frac{1}{6} \zeta+\frac{1}{12} \zeta^{2}\right)^{1 / 2} \frac{3}{2} \tilde{F} \cos \Psi \geq 0 \text {. }
$$

Neglecting the contributions of the last two terms, we have

which gives that

$$
\frac{\zeta}{4}\left(\frac{1}{4} \zeta-1\right) \geq 0
$$

$$
\zeta=0 \quad \text { or } \quad \zeta \geq 4, \quad \text { i.e., } \quad \delta w=0 \text { or } \frac{\delta w^{2}}{b_{1}} \geq 4 \frac{a_{1}+a_{3}^{2}}{a_{1}}
$$

these are identical to Eq.(263).

These results reveal that in two-phase inviscid flow with non zero relative motions, if the contributions from turbulence are completely ignored, then the model has real characteristics when and only when

The region

$$
\frac{\delta w^{2}}{b_{1}} \geq 4 \frac{a_{1}+a_{3}^{2}}{a_{1}} .
$$

$$
0<\delta w^{2}<4 \frac{a_{1}+a_{3}^{2}}{a_{1}} b_{1}
$$


in which the model possesses complex characteristics will be reduced with the increase of $k_{d}$ and finally disappears when $k_{d}$ is large enough. The required $k_{d}$ that model will have all real characteristics for all $\delta w \geq 0$ can be estimated by solving equation (340). 


\section{Appendix C. The evolution of turbulent kinetic energy}

The evolution equation of ordered kinetic energy $k_{0}$ of a fluid mixture is given by Eq. (55):

$$
0=\rho \frac{d}{d t} k_{o}+\vec{\nabla} \cdot\left(\vec{\sigma}_{o}+\vec{\pi}_{o}\right)+\left(\mathbf{R}_{\mathbf{o}}-\mathbf{Q}\right): \vec{\nabla} \vec{u}+(\vec{\nabla} P) \cdot \vec{w}+\rho \phi_{\circ}+2 \omega \rho k_{\circ}+\rho \epsilon_{\circ} .
$$

In a one-dimensional system consisting of only two fluids,

$$
\begin{gathered}
\overrightarrow{\sigma_{o}}=\sum_{j=1}^{2} \frac{1}{2} \rho x_{j} w_{j}^{3} \hat{\mathrm{x}}=\rho k_{o} a_{2} \delta w \hat{\mathrm{x}}, \\
\vec{\nabla} \cdot \vec{\sigma}_{\circ}=k_{o} a_{2} \delta w \frac{\partial \rho}{\partial x}+\frac{a_{2}^{2}-2 a_{1}}{a_{1}} \rho k_{o} \delta w \frac{\partial x_{1}}{\partial x}+3 a_{2} \rho k_{o} \frac{\partial \delta w}{\partial x},
\end{gathered}
$$

and

$$
\begin{aligned}
\rho \phi_{o} & =\frac{2}{3} \frac{\partial\left(f_{1} \rho k_{d}\right)}{\partial x} w_{1}-\frac{4}{3} \frac{\partial\left(f_{1} \Gamma_{d 1}\right)}{\partial x} w_{1}+\frac{2}{3} \frac{\partial\left(f_{2} \rho k_{d}\right)}{\partial x} w_{2}-\frac{4}{3} \frac{\partial\left(f_{2} \Gamma_{d 2}\right)}{\partial x} w_{2} \\
& =\frac{2}{3} \frac{\partial\left(\rho k_{d}\right)}{\partial x} w+\frac{2}{3} \rho k_{d} \delta w \frac{\partial f_{1}}{\partial x}-\frac{4}{3}\left[w_{1} \frac{\partial\left(f_{1} \Gamma_{d 1}\right)}{\partial x}+w_{2} \frac{\partial\left(f_{2} \Gamma_{d 2}\right)}{\partial x}\right] \\
& =\frac{2}{3} k_{d} w \frac{\partial \rho}{\partial x}+\frac{2}{3} \rho w \frac{\partial k_{d}}{\partial x}+\frac{2}{3} \rho k_{d} \delta w \frac{\partial f_{1}}{\partial x}-\frac{4}{3}\left[w_{1} \frac{\partial\left(f_{1} \Gamma_{d 1}\right)}{\partial x}+w_{2} \frac{\partial\left(f_{2} \Gamma_{d 2}\right)}{\partial x}\right] .
\end{aligned}
$$

For our purpose, we now consider inviscid flow, i.e., $\eta=0, \rho \epsilon_{\circ}=0$, and further assume that the turbulent viscosity is neglegible $\left(\eta_{d}=0\right.$ and $\left.\Gamma_{d j}=0\right)$. Thus the one-dimensional $k_{\text {o }}$ equation becomes

$$
\begin{gathered}
\rho \frac{d k_{\circ}}{d t}+\left(a_{2} k_{\circ}+\frac{2}{3} a_{3} k_{d}\right) \delta w \frac{\partial \rho}{\partial x}+2 \rho k_{\circ} \frac{\partial u}{\partial x}+\frac{a_{2}^{2}-2 a_{1}}{a_{1}} \rho k_{\circ} \delta w \frac{\partial x_{1}}{\partial x}+3 a_{2} \rho k_{\circ} \frac{\partial \delta w}{\partial x} \\
+\frac{2}{3} \rho a_{3} \delta w \frac{\partial k_{d}}{\partial x}+a_{3} \delta w \frac{\partial P}{\partial x}+\frac{2}{3} \rho k_{d} \delta w \frac{\partial f_{1}}{\partial x}+2 \omega \rho k_{\circ}=0
\end{gathered}
$$

where we have used $w=a_{3} \delta w$ and $\vec{\pi}_{o}=0$. Combining this equation with the onedimensional $k_{d}$ equation (105) for the case $\eta=0=\eta_{d}$,

$$
\begin{gathered}
\rho \frac{d k_{d}}{d t}+k_{d} a_{3} \delta w \frac{\partial \rho}{\partial x}+\frac{2}{3} \rho k_{d} \frac{\partial u}{\partial x}-\frac{5}{3} \rho k_{d} \delta w \frac{\partial x_{1}}{\partial x}+\frac{5}{3} \rho k_{d} a_{3} \frac{\partial \delta w}{\partial x}+ \\
\rho a_{3} \delta w \frac{\partial k_{d}}{\partial x}+\frac{\partial J_{k}}{\partial x}+\rho k_{d} \delta w \frac{\partial f_{1}}{\partial x}-\omega \rho a_{1} \delta w^{2}+\rho \epsilon_{d}=0
\end{gathered}
$$

and defining

$$
\psi \equiv \frac{k_{d}}{k_{o}}
$$

gives

$$
\frac{\rho}{\psi} \frac{d \psi}{d t}=\frac{\rho}{k_{d}} \frac{d k_{d}}{d t}-\frac{\rho}{k_{\circ}} \frac{d k_{\circ}}{d t}
$$


that is

$$
\begin{aligned}
\frac{\rho}{\psi} \frac{d \psi}{d t}=- & \delta w\left[a_{3}\left(1-\frac{2}{3} \psi\right)-a_{2}\right] \frac{\partial \rho}{\partial x}+\frac{4}{3} \rho \frac{\partial u}{\partial x}+\rho \delta w\left(\frac{5}{3}+\frac{a_{2}^{2}-2 a_{1}}{a_{1}}\right) \frac{\partial x_{1}}{\partial x}- \\
& \rho\left(\frac{5}{3} a_{3}-3 a_{2}\right) \frac{\partial \delta w}{\partial x}-\rho\left(1-\frac{2}{3} \psi\right) \frac{a_{3} \delta w}{k_{d}} \frac{\partial k_{d}}{\partial x}-\frac{1}{k_{d}} \frac{\partial J_{k}}{\partial x}+ \\
& \frac{a_{3} \delta w}{k_{o}} \frac{\partial P}{\partial x}-\rho \delta w\left(1-\frac{2}{3} \psi\right) \frac{\partial f_{1}}{\partial x}+2 \omega \rho\left(1+\frac{1}{\psi}\right)-\rho \frac{\varepsilon_{d}}{k_{d}} \\
=- & \delta w\left[a_{3}\left(1-\frac{2}{3} \psi\right)-a_{2}\right] \frac{\partial \rho}{\partial x}+\frac{4}{3} \rho \frac{\partial u}{\partial x}+\rho \delta w\left(\frac{5}{3}+\frac{a_{2}^{2}-2 a_{1}}{a_{1}}\right) \frac{\partial x_{1}}{\partial x}- \\
& \rho\left(\frac{5}{3} a_{3}-3 a_{2}\right) \frac{\partial \delta w}{\partial x}-\rho a_{3} \delta w\left(1-\frac{2}{3} \psi\right) \frac{\partial \ln k_{d}}{\partial x}+\frac{1}{k_{d}} \frac{\partial}{\partial x}\left(D_{k} \frac{\partial k_{d}}{\partial x}\right)+ \\
& \frac{a_{3} \delta w}{k_{\circ}} \frac{\partial P}{\partial x}-\rho \delta w\left(1-\frac{2}{3} \psi\right) \frac{\partial f_{1}}{\partial x}+\omega \rho\left(1+\frac{2}{\psi}\right)
\end{aligned}
$$

where we have used $\epsilon_{d}=\omega k_{d}$. For tiny $\delta w$, Eq.(348) becomes

$$
\frac{\rho}{\psi} \frac{d \psi}{d t} \sim \frac{4}{3} \rho \frac{\partial u}{\partial x}+\frac{1}{k_{d}} \frac{\partial}{\partial x}\left(D_{k} \frac{\partial k_{d}}{\partial x}\right)+\omega \rho\left(1+\frac{2}{\psi}\right) .
$$

From equation (349), we see that for both $\delta w$ and $\psi$ initially tiny, $\frac{d \psi}{d t}=2 \omega>0$, so $\psi$ grows with time unless $\omega=0$ or both $k_{o}$ and $k_{d}$ vanish at the same time. Note that $D_{k} \sim \eta_{d} \approx 0$ in our discussion, while $\frac{4}{3} \frac{\partial u}{\partial x}=-\frac{d}{d t}\left(\ln \rho^{4 / 3}\right)$, thus equation (349) can be rewritten as

$$
\frac{d}{d t} \ln \left(\psi \rho^{4 / 3}\right) \sim \omega\left(1+\frac{2}{\psi}\right) \geq 0 \text { when } \delta w \text { is tiny. }
$$

Therefore we conclude that $k_{d}$ and $k_{o}$ in inviscid flows are generated simultaneously by any nonzero relative motion between the fluids. As long as $k_{d}$ and $\eta_{d}$ are kept nonzero, as shown in Section 4, the hybrid turbulent mix model will always have real characteristics.

Meanwhile, it is worthwhile to point out that $\psi=$ constant or a function of only $x_{1}$ and $x_{2}$ are not solutions to equation (348). Therefore the relationship between $k_{d}$ and $k_{0}$ is much more complicated than the one recently proposed by Ramshaw ${ }^{[15]}$. The real relationship between them only can be determined by solving Eq.(348).

X-HM, MS D413, Los Alamos National Laboratory, Los alamos, NM 87544 